\title{
Detection of Special Operations Forces Using Night Vision Devices
}

September 2001

Cyrus M. Smith 


\title{
DOCUMENT AVAILABILITY
}

Reports produced after January 1, 1996, are generally available free via the U.S. Department of Energy (DOE) Information Bridge:

Web site: http://www.osti.gov/bridge

Reports produced before January 1, 1996, may be purchased by members of the public from the following source:

\author{
National Technical Information Service \\ 5285 Port Royal Road \\ Springfield, VA 22161 \\ Telephone: 703-605-6000 (1-800-553-6847) \\ TDD: 703-487-4639 \\ Fax: 703-605-6900 \\ E-mail: info@ntis.fedworld.gov \\ Web site: http://www.ntis.gov/support/ordernowabout.htm
}

Reports are available to DOE employees, DOE contractors, Energy Technology Data Exchange (ETDE) representatives, and International Nuclear Information System (INIS) representatives from the following source:

Office of Scientific and Technical Information

P.O. Box 62

Oak Ridge, TN 37831

Telephone: 865-576-8401

Fax: 865-576-5728

E-mail: reports@adonis.osti.gov

Web site: http://www.osti.gov/contact.html

This report was prepared as an account of work sponsored by an agency of the United States Government. Neither the United States government nor any agency thereof, nor any of their employees, makes any warranty, express or implied, or assumes any legal liability or responsibility for the accuracy, completeness, or usefulness of any information, apparatus, product, or process disclosed, or represents that its use would not infringe privately owned rights. Reference herein to any specific commercial product, process, or service by trade name, trademark, manufacturer, or otherwise, does not necessarily constitute or imply its endorsement, recommendation, or favoring by the United States Government or any agency thereof. The views and opinions of authors expressed herein do not necessarily state or reflect those of the United States Government or any agency thereof. 
ORNL/TM-2001/172

\title{
DETECTION OF SPECIAL OPERATIONS FORCES USING NIGHT VISION DEVICES
}

\author{
Cyrus M. Smith
}

September 2001

\author{
Prepared by \\ OAK RIDGE NATIONAL LABORATORY \\ P. O. Box 2008 \\ Oak Ridge, Tennessee 37831-6285 \\ managed by \\ UT-Battelle, LLC \\ for the \\ U.S. DEPARTMENT OF ENERGY \\ under contract DE-AC05-00OR22725
}





\section{CONTENTS}

\section{Page}

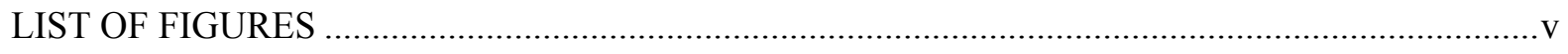

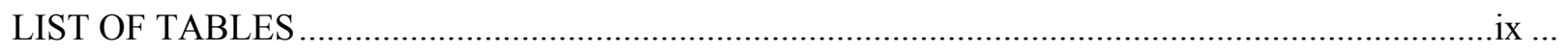

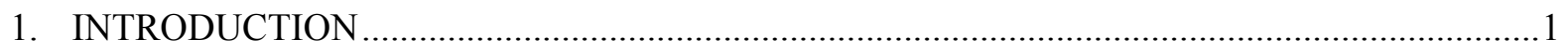

2. UNAIDED NIGHT VISION CONSIDERATIONS ….........................................................

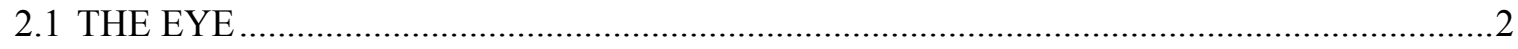

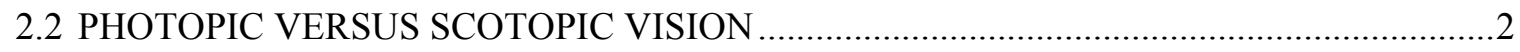

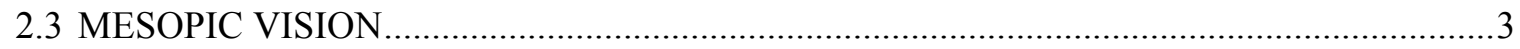

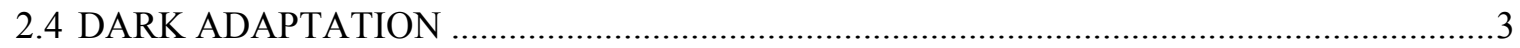

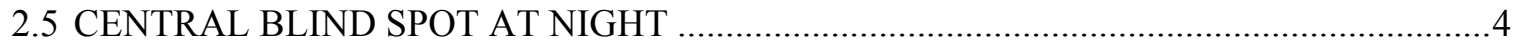

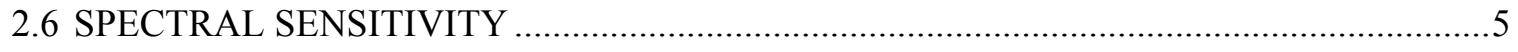

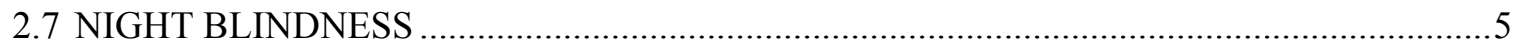

3. NIGHT VISION DEVICES …

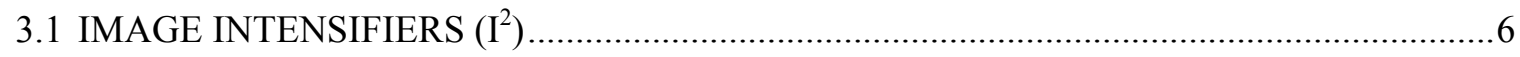

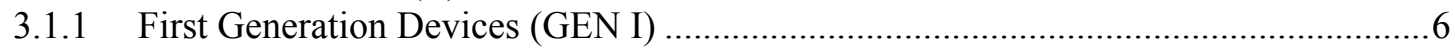

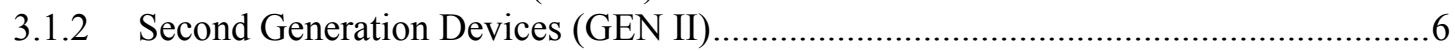

3.1.3 Third Generation Devices (GEN III) .............................................................

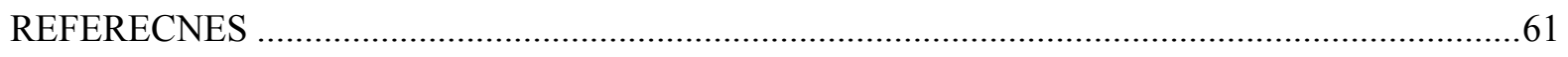

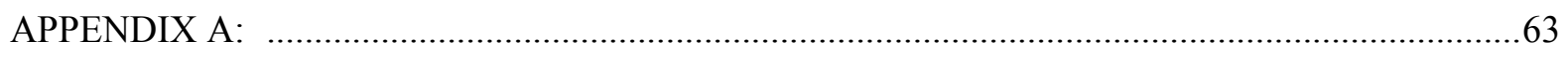

APPENDIX B: 



\section{LIST OF FIGURES}

Figure

Page

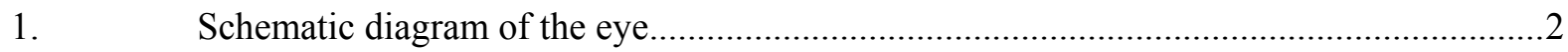

2. Brightness range over which the human eye can adequately function............................. 3

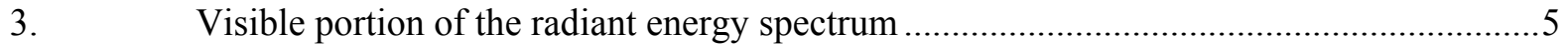

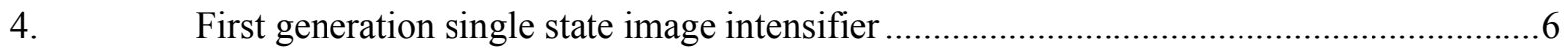

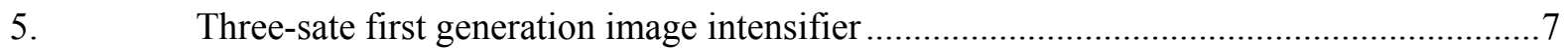

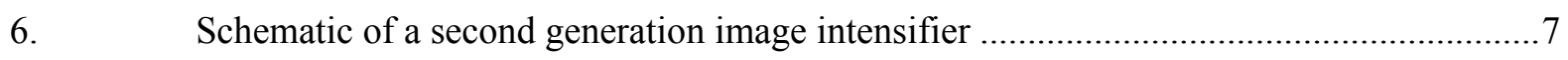

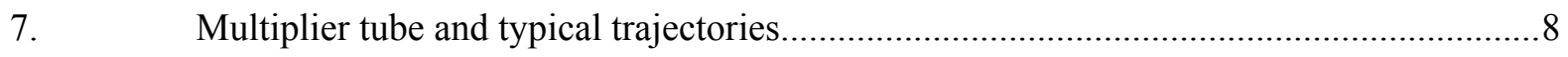

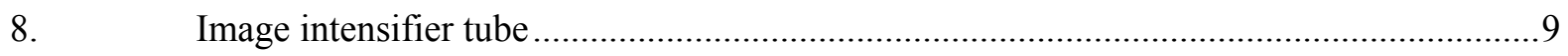

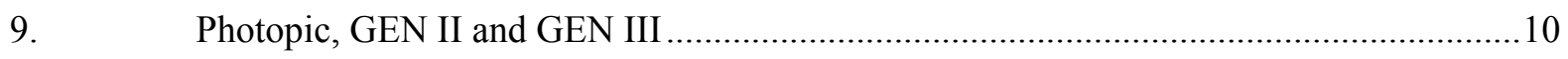

10. Spectral comparison of the GEN II and GEN III image intensifiers and the spectral Distribution of the night sky (starlight and moonlight) ....................................................11

11. Spectral output of GEN II and GEN III image intensifier..........................................11

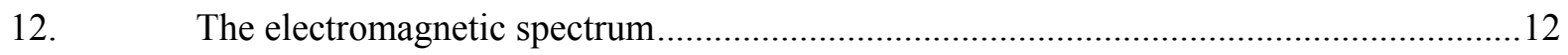

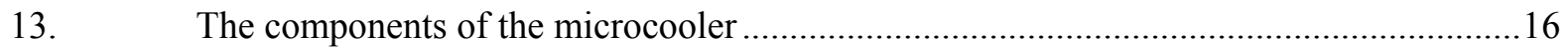

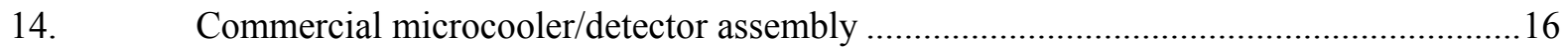

15. Graphical description of photodiode infrared detector ................................................ 17

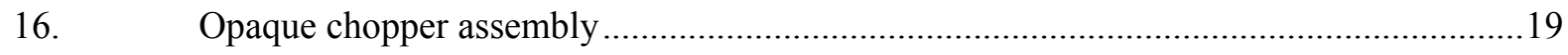

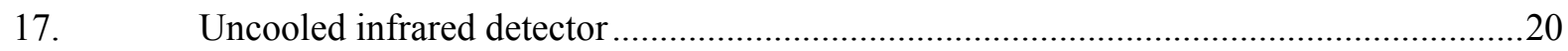

18. Natural luminous incidence (illuminance) as a function of solar and lunar altitude and for various phases of the moon as measured by Brown ..............................................22

19. Night sky spectral radiant sterance for various phse of the moon................................23

20. Detailed night sky radiance in the $450 \mu \mathrm{m}$ to $900 \mu \mathrm{m}$ band ..........................................24 
21. The solar spectral radiant incidence (irradiance) at sea level for one air absorption by atmospheric constituents is indicated by shaded areas.

22. Spectral transmittance of atmospheric gases and the solar radiant incidence spectrum....25

23. Typical atmospheric transmittance spectrum

24. Atmospheric transmittance spectrum. Shows carbon dioxide $\left(\mathrm{CO}_{2}\right)$ and water $\left(\mathrm{H}_{2} \mathrm{O}\right)$ absorption bands

25. Computed spectra of atmospheric transmittance for five model atmospheres with $23 \mathrm{~km}$ horizontal visibility

26. Computed spectra of atmospheric transmittance for five model atmospheres with $5 \mathrm{~km}$ horizontal visibility

27. Atmospheric transmittance spectra for selective fogs .

28. Atmospheric transmittance spectra for haze

29. Average transmittance (8-12 micrometers)

30. Approximate transmittance using absolute humidity

31. Visual acuity as a function of night sky condition and generation of night vision goggle with high contrast targets

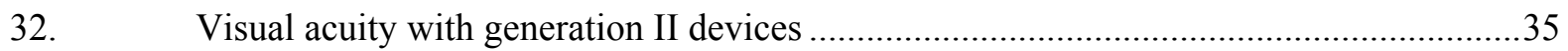

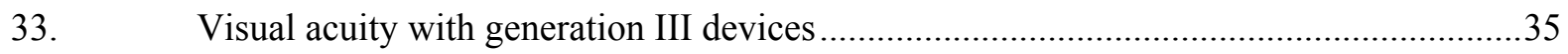

34. Visual acuity as a function of night sky condition and target contrast with generation III night vision goggles .36

35. Visual acuity as a function of contrast and night vision goggle generation under Simulated full moon radiance. 36

36. Visual resolution with simulated full moon ambient luminance and three target contrasts.

37. Visual resolution with simulated quarter moon ambient luminance and two target Contrasts. 38

38. Histogram of canopy site $(05 / 09 / 88)$ . .38 


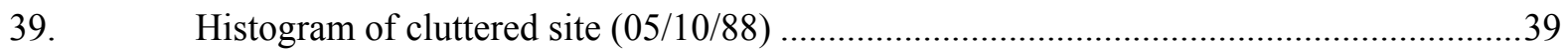

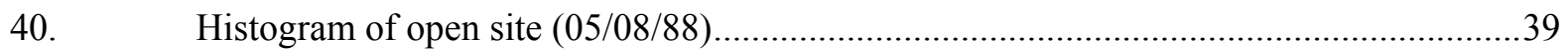

41. Visual acuity SNR for low contrast starlight conditions ............................................

42. Visual acuity versus SNR for high contrast starlight conditions...................................41

43. Visual acuity versus SNR for low contrast quarter moon conditions..............................41

44. Visual acuity versus SNR for high contrast quarter moon conditions ...........................42

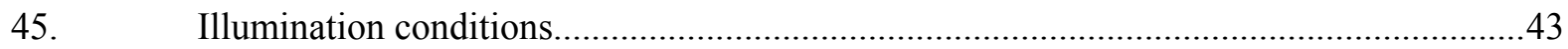

46. Spectral irradiance normalized to 1 foot candle illuminance for night sky (A), mercury lamp (B), fluorescent lamp (C), and foliage reflection (D) .............................................44

47. Low light level range increases of GEN III AN/PVS-7 over AN/PVS-5 as function

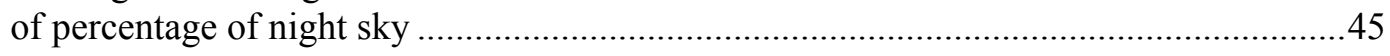

48. Measured resolution of ANVIS-6 versus illumination ..................................................47

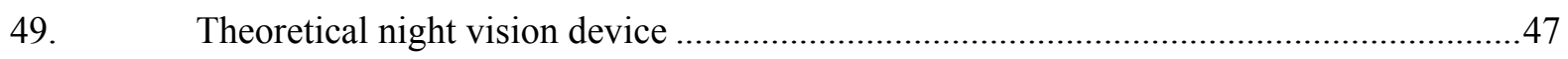

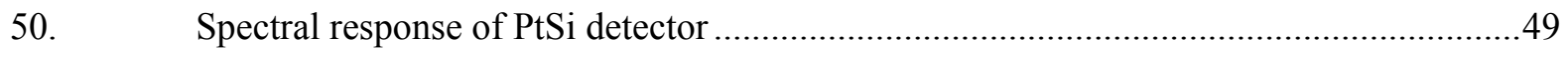

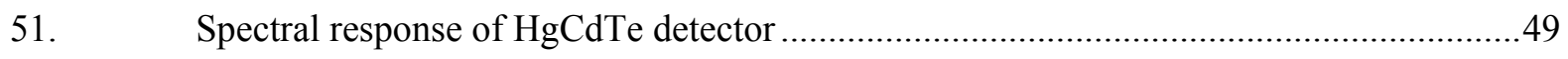

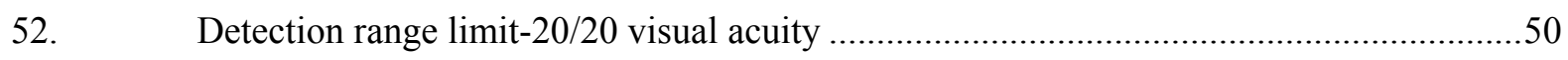

53. Detection of unlighted life rafts (wave height between 3 and 5 feet).............................51

54. Detection of unlighted life rafts (wave height)........................................................52

55. Helicopter detection of personnel in the water (PIW) (visibility $=15 \mathrm{nmi}) \ldots \ldots \ldots \ldots \ldots \ldots \ldots . . . .52$

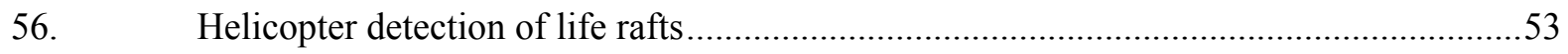

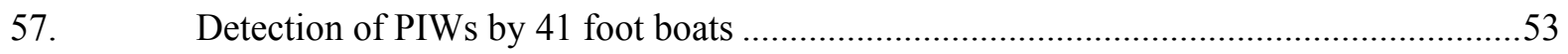

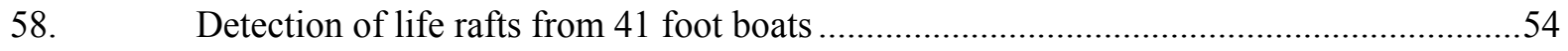

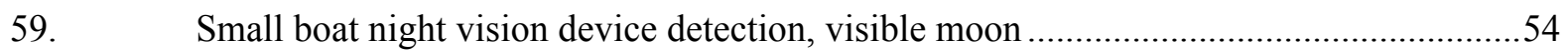

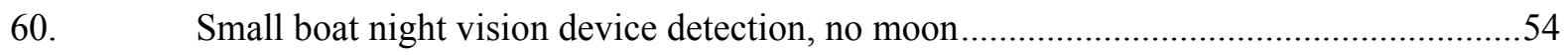


61. Life-raft night vision device detection, visible moon..................................................55

62. Life-raft night vision device detection, no moon .....................................................55

63. Personnel in the water (PIW). Detection with night vision devices, visible moon ..........56

64. Personnel in the water (PIW). Detection by night vision devices, no moon ...................56

65. Mean distance for detection of human targets by infrared imagers.................................58

66. Mean distance for recognition of the human target by infrared imagers..........................59

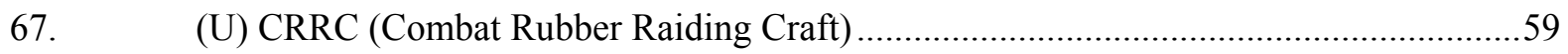

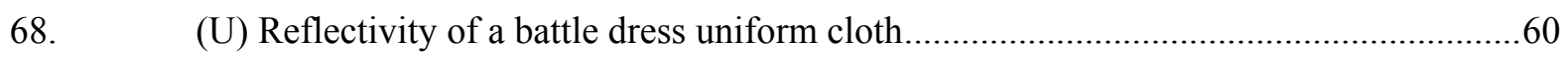

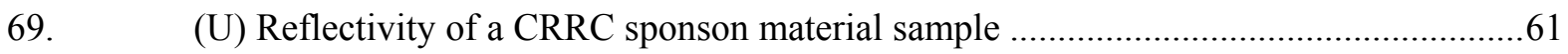




\section{LIST OF TABLES}

Table

Page

1. Comparison on Second Generation (GEN II) and Third Generation (GEN III) Image Intensifiers

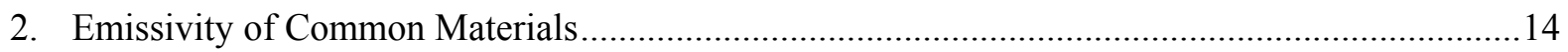

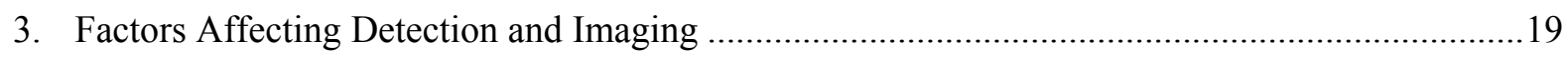

4. Contrast Values for Various Systems, Targets, and Backgrounds for Clear Starlight Illumination 30

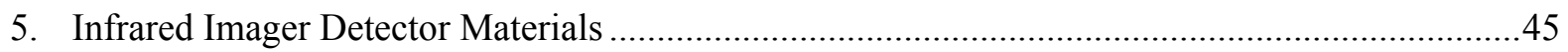

6. Characteristics of Infrared Imager Systems in Rabin, 1994 Study ...............................................54

7. Image Intensifier Based Night Vision Equipment for NRL Study ...........................................57

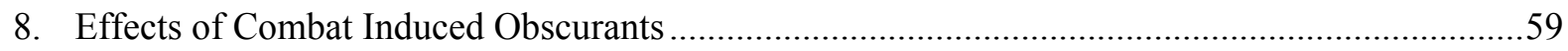





\section{INTRODUCTION}

Night vision devices, such image intensifiers and infrared imagers, are readily available to a host of nations, organizations, and individuals through international commerce. Once the trademark of special operations units, these devices are widely advertised to "turn night into day". In truth, they cannot accomplish this formidable task, but they do offer impressive enhancement of vision in limited light scenarios through electronically generated images.

Image intensifiers and infrared imagers are both electronic devices for enhancing vision in the dark. However, each is based upon a totally different physical phenomenon. Image intensifiers amplify the available light energy whereas infrared imagers detect the thermal energy radiated from all objects. Because of this, each device operates from energy which is present in a different portion of the electromagnetic spectrum. This leads to differences in the ability of each device to detect and/or identify objects.

This report is a compilation of the available information on both state-of-the-art image intensifiers and infrared imagers. Image intensifiers developed in the United States, as well as some foreign made image intensifiers, are discussed. Image intensifiers are categorized according to their spectral response and sensitivity using the nomenclature of GEN I, GEN II, and GEN III. As the first generation of image intensifiers, GEN I, were large and of limited performance, this report will deal with only GEN II and GEN III equipment. Infrared imagers are generally categorized according to their spectral response, sensor materials, and related sensor operating temperature using the nomenclature Medium Wavelength Infrared (MWIR) Cooled and Long Wavelength Infrared (LWIR) Uncooled. MWIR Cooled refers to infrared imagers which operate in the 3 to $5 \mu \mathrm{m}$ wavelength electromagnetic spectral region and require either mechanical or thermoelectric coolers to keep the sensors operating at $77^{\circ} \mathrm{K}$. LWIR Uncooled refers to infrared imagers which operate in the 8 to $12 \mu \mathrm{m}$ wavelength electromagnetic spectral region and do not require cooling below room temperature. Both commercial and military infrared sensors of these two types are discussed.

There is an abundance of information available on both image intensifiers and infrared imagers in the classified and unclassified literature. However, it is the objective of this report to compile the relevant portions of this information into a single reference document for the special operations operator. This report provides the operator with knowledge which will assist him in accessing his vulnerability to detection by night vision devices. First, a simplified explanation of physical parameters affecting unaided night vision will be given. Secondly, the various categories of image intensifiers and infrared imagers and their performance parameters will be discussed. Thirdly, the various real world factors which influence the detection performance of image intensifiers and infrared imagers will be explained. Next, the results of studies on the ability of night vision devices to detect and identify special operations related watercraft and swimmers will be evaluated. Finally, in an attempt to address the question "What does this technology mean in terms of me being detected?" A summary of the detection limits of these devices and practical recommendations and guidelines for the operator will be proposed. 


\section{UNAIDED NIGHT VISION CONSIDERATIONS}

The effects of decreased illumination on vision can be dramatic. ${ }^{1}$ Visual acuity may be reduced to 20/200 or less, color vision is lost, blue-green lights will appear brighter while red lights will appear dimmer, depth perception is degraded, glare is a factor, and a central blind spot is present.

\subsection{The Eye}

The performance characteristics of the human eye are a very complex subject which has undergone a wide variety of studies for many years. It is unfortunate that the eye is not a constant among individuals. The limiting resolution of the eye is very dynamic and varies with brightness, contrast, dark adaptation time, flicker rate, viewing time, color content, target movement, clutter, spatial and temporal noise, axial locations of the target relative to the axis of the eye, and many other physiological conditions.

The basic structure of the eye is shown in Figure 1. The front of the eye is a lens system which is comprised of the cornea and crystalline lens. Their purpose is to focus the light onto the retina. The retina contains receptor cells, rods and cones, which, when stimulated by light, send signals to the brain. These signals are subsequently interpreted as vision.

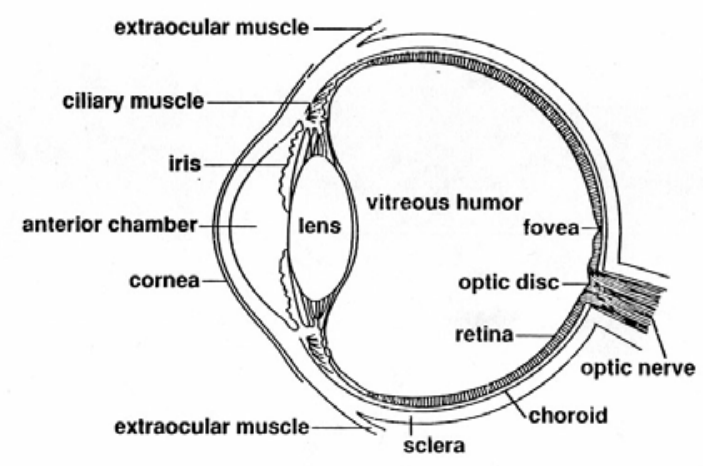

Fig. 1. Schematic diagram of the eye.

Most of the receptors are rods, which are mostly found in the periphery of the retina. The cones are located mostly in the center and middle of the retina. Although there are approximately 17 rods for every cone, the cones, concentrated in the center, allow resolution of fine detail and color discrimination. The rods cannot distinguish colors and have poor resolution, but they have a much higher sensitivity to light than the cones.

\subsection{Photopic Versus Scotopic Vision}

The rods are responsible for vision under very dim levels of illumination (scotopic vision) and the cones function at higher illumination levels (photopic vision). Photopic vision provides the capability to see color and resolve fine detail (20/20 or better), but it functions only in good illumination. Scotopic vision is of poorer quality; it is limited by reduced resolution (usually 20/200 or less) and provides the ability to discriminate only between shades of black and white. The scotopic system provides enhanced sensitivity and low detection thresholds under reduced illumination. 
The system of rods and cones allows the eye to maintain sensitivity over a large range of light levels as illustrated in Figure 2. The sensitivity of the eye automatically adjusts to changes in illumination. The dimmest light in which rods can function is equivalent to conditions of an overcast night with no moonlight. The dimmest light in which cones function is roughly equivalent to a night with $50 \%$ moonlight.

\subsection{Mesopic Vision}

There is a common misconception that the rods are used only at night and the cones only during the day. Actually, both rods and cones function over a wide range of light levels and, at intermediate levels, they function simultaneously. The transition zone between photopic and scotopic vision, where the level of illumination is equivalent to twilight or dusk, is call esopic vision. Neither the rod nor the cones operate at peak efficiency in this range, but both actively contribute to visual perception. Mesopic vision is important because the brightness output from Night Vision Goggles (NVG) falls into this range.

\subsection{Dark Adaptation}

Dark adaptation is a process during which each eye independently adjusts from a high-luminance setting to a low-luminance setting. Both rods and cones contain light-sensitive chemicals called photopigments. The photopigment in the rods is called rhodopsin, or visual purple. There are three types of cone photopigments (red, blue, and green sensititive) that are chemically only slightly different from visual purple. Upon exposure to light, photopigments undergo a chemical reaction that converts light energy to electrical activity, initiating visual impulses in the retina that are conducted by nerve fibers from the eyes to the brain. Exposure to intense light decomposes the photopigments and reduces retinal sensitivity to dim light. The photopigments are regenerated during dark adaptation.

The fully dark-adapted eye restores retinal sensitivity to its maximal level. Rods and cones differ in their rate of dark adaptation. Cones attain maximum sensitivity in 5 to 7 minutes, while rods require 30 to 45 minutes or longer of absolute darkness to attain maximum sensitivity after exposure to bright light. Retinal sensitivity is very low when darkness is first encountered, but the sensitivity increases 10 times within 1 minute. After 20 minutes, sensitivity has increased 6,000 times, and after 40 minutes, sensitivity has

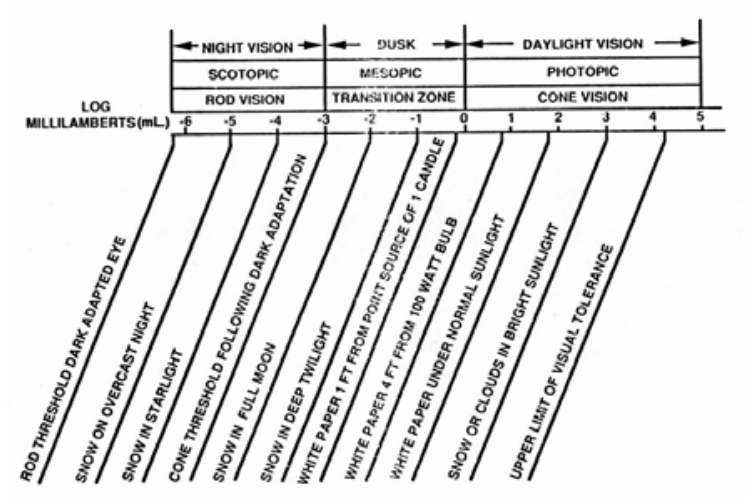

Fig. 2. Brightness range over which the human eye can adequately function 
increased 25,000 times. The initial rapid increase in sensitivity is caused by the rapid adaptation of the cones, which have a faster rate of photochemical regeneration. The rods are more slowly to adapt, but eventually achieve a much greater sensitivity than the cones. In general, dark adaptation is about $80 \%$ complete within 30 minutes, but it may take hours, or even days to acquire total dark adaptation.

In addition to changes in photopigment concentrations, the eye has other mechanisms for adapting to changing light conditions. Dark adaptation is also affected by physical changes in the size of the pupil. The diameter of the pupil can contract to $1.5 \mathrm{~mm}$ and expand to $8 \mathrm{~mm}$, which equates to a 30 times change in the amount of light entering the eye. Another light-adaptive mechanism is neural adaptation. When light intensity first decreases, the intensities of the signals transmitted by the retinal neurons are very low. However, the intensities of these signals increase rapidly over time. This change in "neural gain" occurs in seconds and can improve night vision by a factor of 10 or more. Neural adaptation is like having low-speed and high-speed photographic film available in your camera. A large share of the greater sensitivity of rod dark adaptation is a result of retinal summation. As many as 100 rods converge onto a single ganglion cell (nerve fiber) in the retina, which greatly increases sensitivity. Retinal summation dark adaptation sensitivity changes are instantaneous.

Daytime exposure to ordinary sunlight can produce temporary, but cumulative aftereffects on dark adaptation and night vision. Two to three hours of bright sunlight exposure has been shown to delay the onset of rod dark adaptation by 10 minutes or more, and to decrease the final threshold, so that full night vision sensitivity could not be reached for hours. After 10 days of consecutive days of sunlight exposure, the losses in night vision sensitivity were reported to cause a 50\% loss of visual acuity, visibility range, and contract discrimination. Repeated daily exposures to sunlight prolong the time to reach normal scotopic sensitivity so that eventually normal rod sensitivity may not be reached, even overnight.

Dark sunglasses that transmit only $10 \%$ to $16 \%$ of the visible light will prevent degradation of night vision. For sunglasses to be effective, all visible light has to be attenuated, not just portions of the visible spectrum. Thus, colored or yellow visors and glasses are not protective. Dark sunglasses with a neutral gray time are recommended. These sunglasses should have a visible luminance transmission of $15 \%$ or less.

\subsection{CENTRAL BLIND SPOT AT NIGHT}

The fovea, which is the portion of the retina which is responsible for the keenest visual acuity, corresponds to the center of the visual field. The center of the fovea possesses a high density of cones, but it is completely devoid of rods. Thus, if the ambient light is below cone threshold (dim starlight), a central blind spot occurs at the center of the fovea which covers the central 1 degree of the visual field. Each eye also has a physiological blind spot, which is always present. It is caused by the position of the optic nerve at the rear of the eye. There are no rods or cones overlying the optic nerve. Because these physiological blind spots occur at different locations in each eye, they are not apparent when both eyes are open. However, under low light conditions, the central blind spot affects vision. To best detect targets under low light conditions, when only rods are sensitive, an individual must look approximately 15 to 20 degrees to some side of an object in order to place that object's image on the retina in an area which possesses the highest density of rods. 


\subsection{SPECTRAL SENSITIVITY}

The eye is sensitive to wavelengths of light between about $380 \mathrm{~nm}$ to about $760 \mathrm{~nm}$ which is referred to as visible light as shown in Figure 3. Rods and cones are not equally sensitive to these visible wavelengths of light. Unlike the cones, rods are more sensitive to blue light and are not sensitive to wavelengths greater than about $640 \mathrm{~nm}$, the red portions of the visible spectrum. Cones are more sensitive to yellow and red. The most sensitive wavelength for cones is $555 \mathrm{~nm}$ (yellow-green). The most sensitive wavelength for rods is $505 \mathrm{~nm}$ (blue-green). Thus, blue-green lights will generally look brighter at night than red lights. This is one reason why the phosphors in the image intensifiers operate in the green portion of the visible spectrum. The relatively greater brightness of blue or green light, compared with yellow or red light, upon shifting from photopic to scotopic vision is called the Purkinje phenomenon.

\section{THE ELECTROMAGNETIC SPECTRUM}

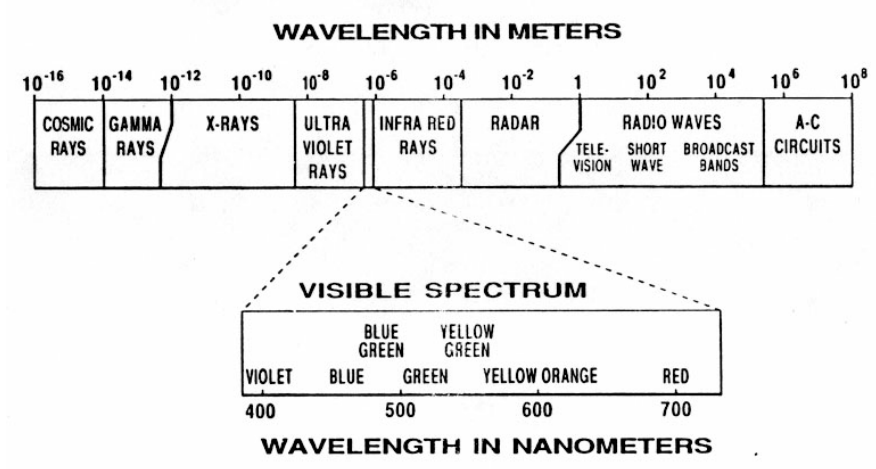

Fig. 3. Visible portion of the radiant energy spectrum.

\subsection{NIGHT BLINDNESS}

True night blindness is unusual. Night blindness can be caused by long-term vitamin A deficiency such as may occur from chronic starvation, alcoholism, deficient fat absorption, and diseases of the liver. Retinal conditions that may cause night blindness are glaucoma, progressive cone/rod dystrophies, drug toxicity, and congenital stationary night blindness. Night vision also decreases with increasing age of individuals.

\section{NIGHT VISION DEVICES}

Man's inability to see in low illumination has always limited his ability to function during the hours of darkness. ${ }^{2}$ History shows us that this is especially true for men in military combat. The United States began experimenting with night vision devices during World War II in hopes of solving this difficulty. They began by introducing a device that would allow them to illuminate an object or area, without being detected by the naked eye. They accomplished this by using the near infra-red wavelength of light. This spectrum is invisible to the naked eye, yet can be seen when viewing through a near infra-red viewing 
device, called the metascope, which is the forerunner of image intensifiers. The army procured large numbers of infra-red lights and metascopes for use in night operations and used them with some success during the Korean conflict. As the years passed, the infra-red lights and metascopes began being used by many countries, to the point, that using infra-red light to illuminate the battlefield was almost as dangerous as using visible light.

\subsection{IMAGE INTENSIFIERS $\left(\mathbf{I}^{2}\right)$}

In 1965, a new device was introduced called the "starlight" scope. This device was compact, passive, lightweight, sturdy, and small enough to be efficiently used as a rifle scope. It improved visibility during periods of low light levels of illumination by amplifying the moonlight and starlight illuminating an area. It could also be used with the old near infra-red search lights. This new capability was a major breakthrough in night vision technology and was due to the introduction of a component called the Image Intensifier $\left(\mathrm{I}^{2}\right)$.

\subsubsection{First Generation Devices (GEN I)}

The first generation image intensifier was a single stage image intensifier tube as shown in Figure 4. The low light level image impacts the fiber optic faceplate. The faceplate transmits the image to the photocathode, where the image is converted into an electronic one. The electronic image is accelerated toward the phosphor screen as it passes through the anode and focusing cone. Actual image intensification takes place during this passage through the anode and focusing cone, and the image is inverted so that an erect amplified image strikes the phosphor screen. Electron gains in the single stage tube are of the order of 50 to 100 times. A three stage tube was also used in first generation image intensifiers which gave higher electron gains. This three stage image intensifier is shown in Figure 5.

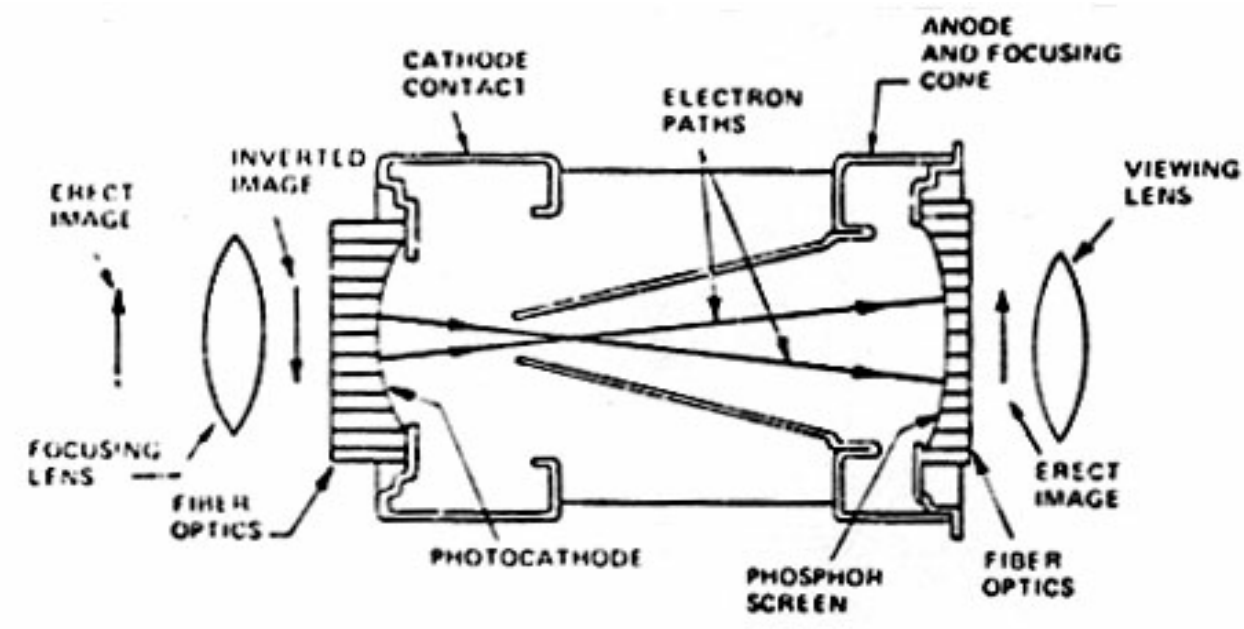

Fig. 4. First generation single state image intensifier.

\subsubsection{Second Generation Devices (GEN II)}

The second generation image intensifiers are identified by their usage of a microchannel plate (MCP). A 
schematic of a typical second generation image intensifier tube is shown in Figure 6. The MCP is a disc shaped electron multiplier. Single electrons striking the input of the MCP are multiplied thousands of times through a process called cascaded secondary emission as illustrated in Figure 7. The MCP consists

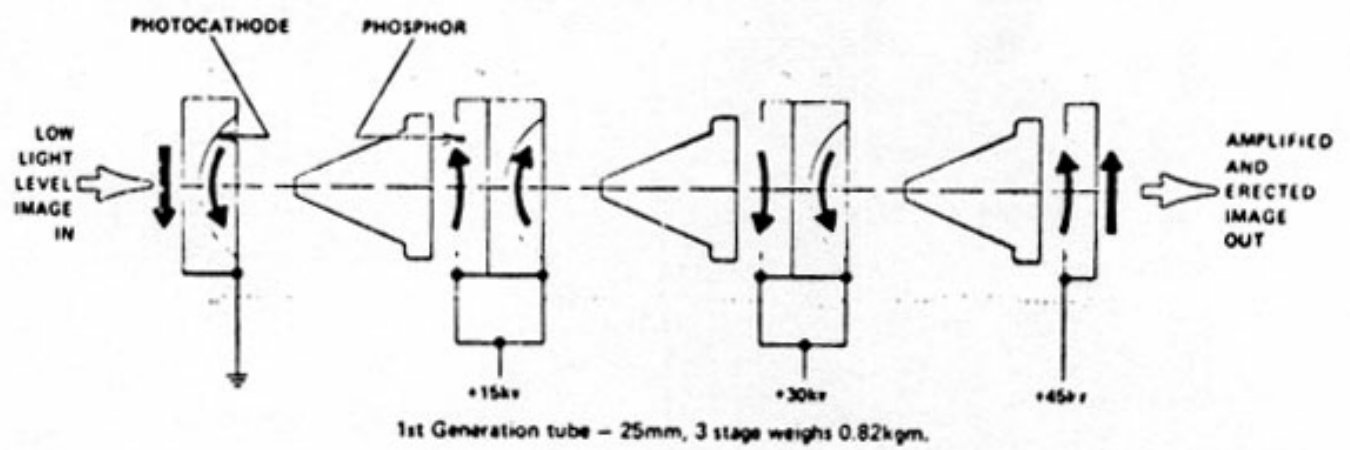

Fig. 5. Three-stage first generation image intensifier.

of millions of microscopic channels or tubes that are fused together into a conducting disc shaped array. These tubes are much finer than a human hair and because each microscopic channel represents a separate high gain electron multiplier, the MCP is an excellent imaging electron multiplier. Because of the tubed channels, perfect position registration is also present between the input and output faces of the MCP for each channel as shown in Figure 8. A disc, similar in size to the diameter of a quarter, and only one third as thick contains approximately 1,7600,000 channels.

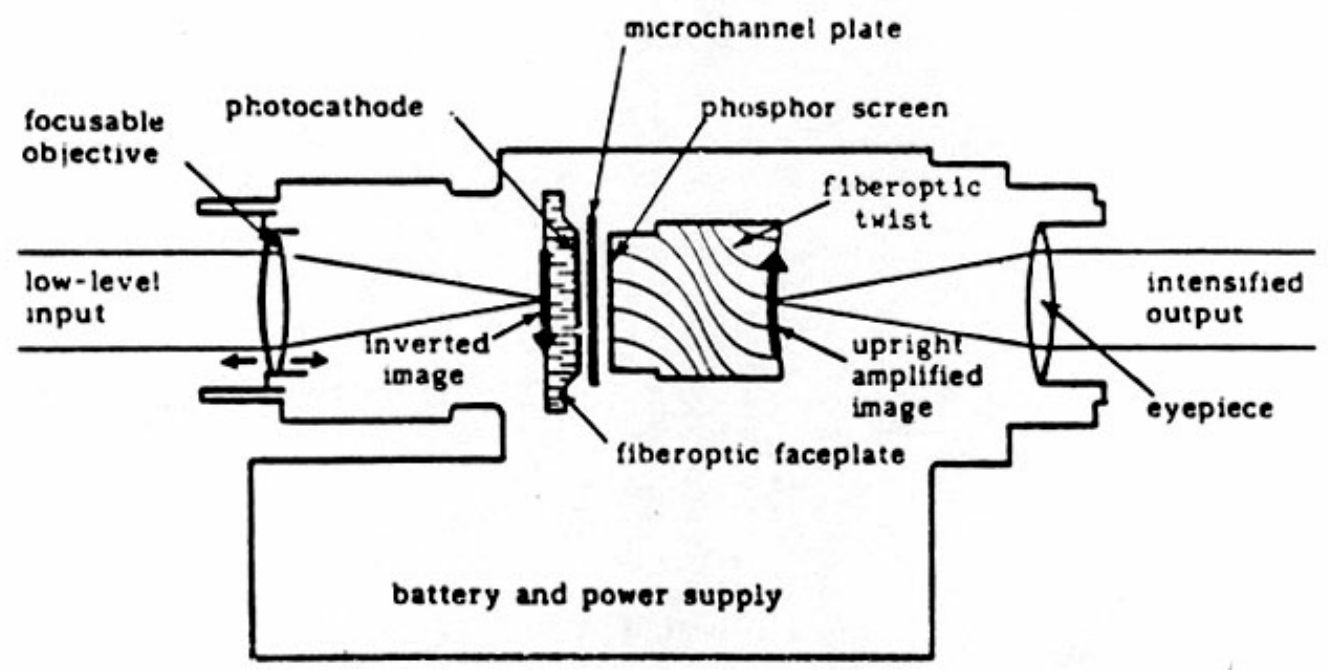

Fig. 6. Schematic of a second generation image intensifier.

Using the MCP, the second generation image intensifier tube is reduced in size by $40 \%$ of the first generation, and the MCP's ability to localize high current regions, resulting from the influence of bright light sources, reduces the blooming and "wash out" problems encountered in the first generation tube. This results in a much better contrast rendition throughout the system, and the second generation tube 
requires less voltage. The second generation tubes, because of their ability to overcome the deficiencies of distortion, vignetting (due to the reduction of stages), phosphor decay, blooming and wash out, remain practical devices for use today. An important feature of modern image intensifiers is the automatic brightness control. The automatic brightness control maintains a controlled voltage and prescribed image brightness. Thus, the automatic brightness control limits the maximum average luminance output of the
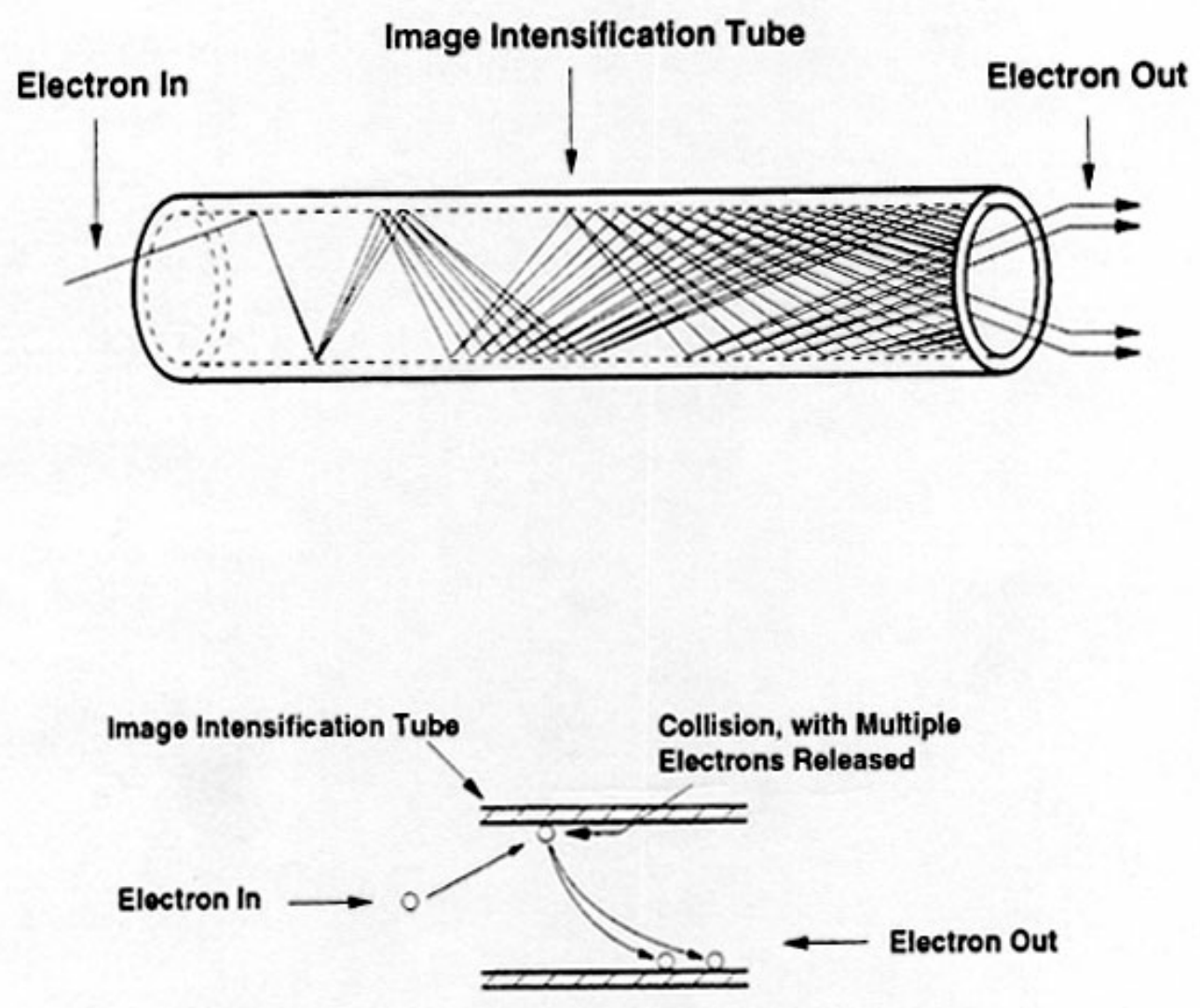

Fig. 7. Multiplier tube and typical trajectories.

phosphor screen. A clamping voltage mechanism protects the photocathode from damage by excessively bright light source, e.g., flares, search lights, fires, explosions, lasers, etc. 


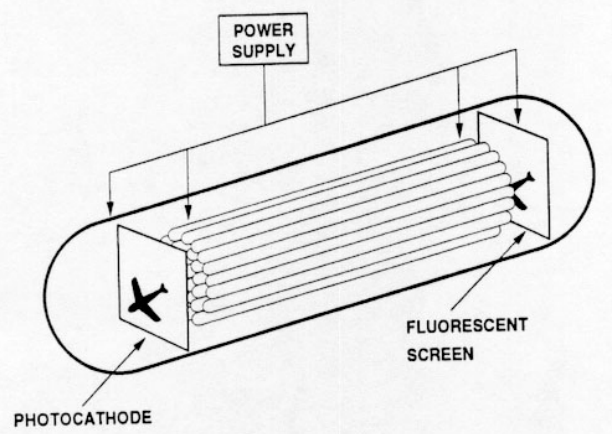

Fig. 8. Image intensifier tube. 


\subsubsection{Third Generation Devices (GEN III)}

The third generation image intensifier is differentiated from a second generation device by higher system amplification, better resolution, larger photocathode response, and better phosphor brightness. Third generation image intensifiers also have longer battery life and are smaller and lighter.

The single most important technical feature of the third generation image intensifier is its improved lowlight performance. The third generation tube outperforms the second generation tube because it has improved efficiency (gain) in the red and IR region of the spectrum. Thus, the image brightness is much greater when viewing objects illuminated by starlight; hence greater system resolution and longer detection ranges. Second and third generation image intensifiers also differ in their spectral response. The response of the second generation tubes is somewhat similar to the human eye ( $380 \mathrm{~nm}$ to $760 \mathrm{~nm}$ ) i.e., it is sensitive to visible light and some IR, ranging from about $400 \mathrm{~nm}$ to $850 \mathrm{~nm}$. The third generation tubes are only sensitive to the orange, red, and near-IR (approximately $625 \mathrm{~nm}$ to $900 \mathrm{~nm}$ ), but it is not sensitive to the blue-green-yellow portion of the spectrum. The spectral sensitivity of a photometer, which corresponds to the human eye, a GEN II image intensifier, and a GEN III image intensifier are all shown in Figure 9. Figure 10 shows this same information with the spectral content of moonlight and starlight added. Since the GEN III imagers are less responsive to the blue-green-yellow portion of the spectrum, they are being used in aircraft which use blue-green cockpit lighting. By adding a minus blue filter to the GEN III device, it becomes virtually blind to blue-green light. On the other hand, the response of the human eye is greatest at night in the blue-green region, making cockpit instruments easy to read even at very low intensities.

Image intensifiers utilize a phosphor screen to produce an amplified light image. Second generation imagers utilize an S-20 phosphor, while third generation imagers utilized a GaAs phosphor. Both These phosphors have a relatively narrow light output band which peaks at $0.53 \mu \mathrm{m}$. Thus the image is green, and color discrimination of objects is not possible. The output spectral response of these phosphors is shown in Figure 11.

\section{Infrared Imagers}

The use of infrared imagers has been highly successful in military, law enforcement, and commercial applications, but has been very limited to date because of the high cost of the equipment. Infrared imagers are true thermal imaging devices that create pictures based on heat energy emitted by the viewed scene rather than light reflected off of it. Heat in the infrared region of the electromagnetic spectrum behaves much like visible light and can be optically focused and collected. Infrared imagers employ various materials whose electrical properties change when heated to perform the transformation from infrared energy to electronic signal levels. These changing signal levels are translated into video signals in which a different shade of gray (or color) on the display is assigned for each detectable temperature (i.e., emitted energy level). Because the energy being sensed is heat and not light, infrared imagers are totally unaffected by the amount of light in a scene. The ability to sense differences in temperature can often provide valuable intelligence above and beyond the ability to see things in the dark.

\section{Infrared Spectrum}

Infrared imagers make use of the infrared electromagnetic spectral band as shown in Figure 12. At the short-wavelength end, the boundary lies at the limit of visual perception, in the deep red. At the longwavelength end, it merges with the "microwave" radio wavelengths, in the millimeter range. The infrared band is often further subdivided into four smaller bands, the boundaries of which are arbitrarily chosen. They include: the "near infrared" $(0.75-3 \mu \mathrm{m})$, the "middle infrared" $(3-6 \mu \mathrm{m})$, and the "far infrared" 
(6 - $15 \mu \mathrm{m})$, and the "extreme infrared" $(15-100 \mu \mathrm{m})$. Although the wavelengths are given in $\mu \mathrm{m}$ (micrometers), other units are often still utilized to measure wavelength in this spectral region, e.g., microns $(\mu)$, manometers $(\mathrm{nm})$ and Angstroms (A).

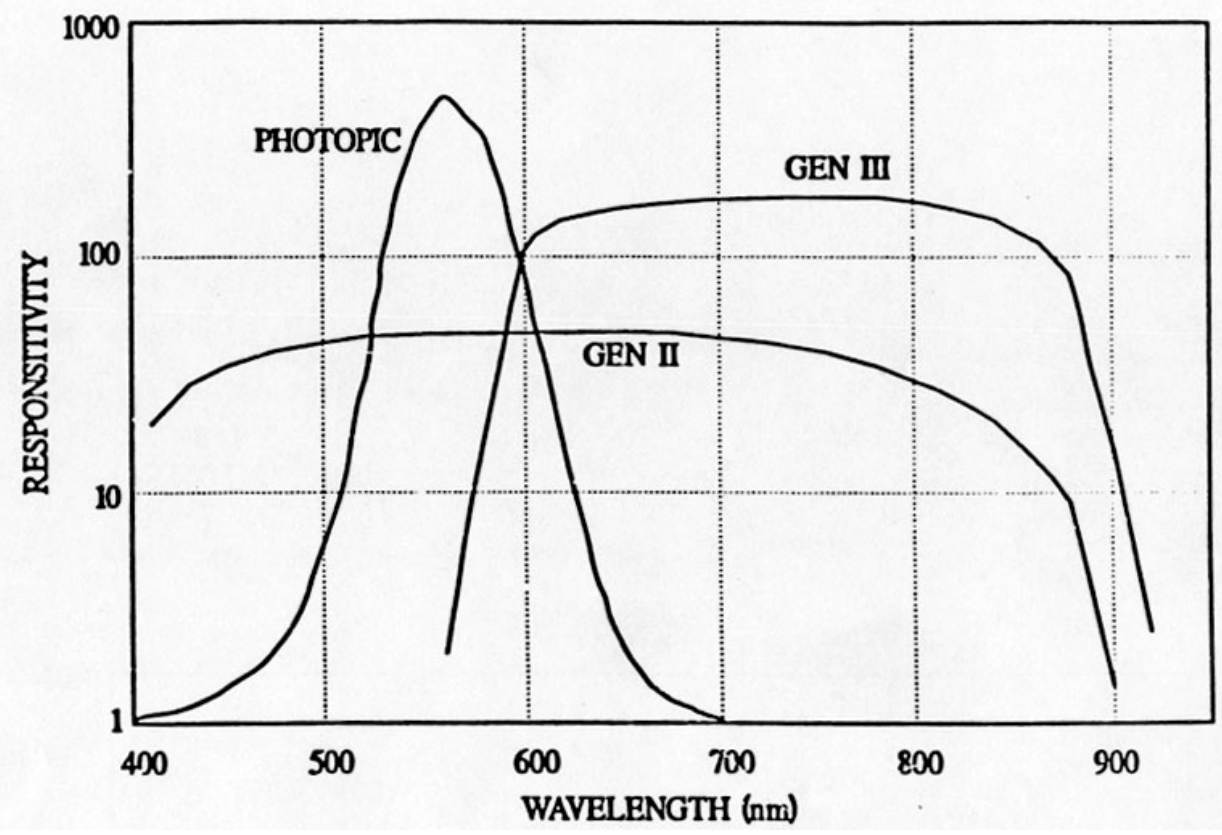

Fig. 9. Photopic, GEN II and GEN III.

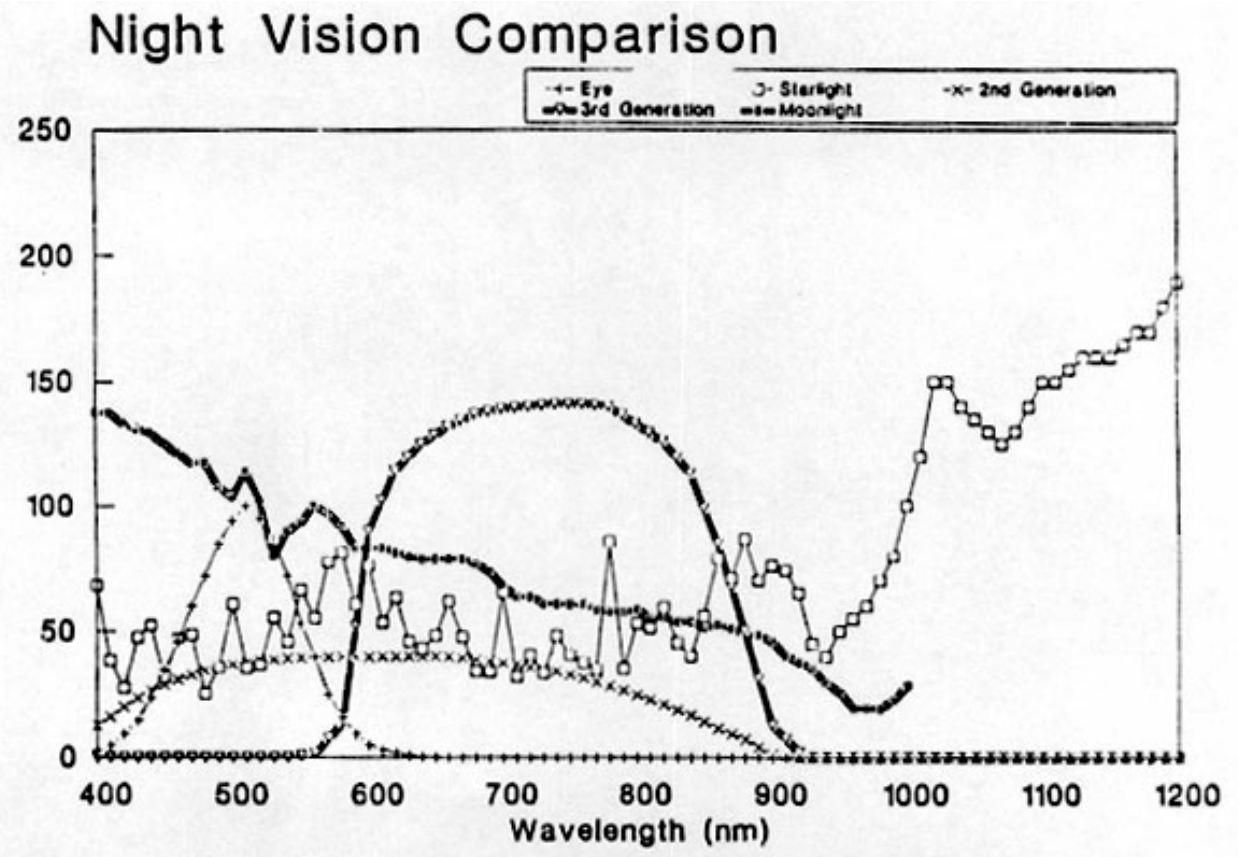


Fig. 10. Spectral comparison of the GEN II and GEN III image intensifiers and the spectral distribution of the night sky (starlight and moonlight).

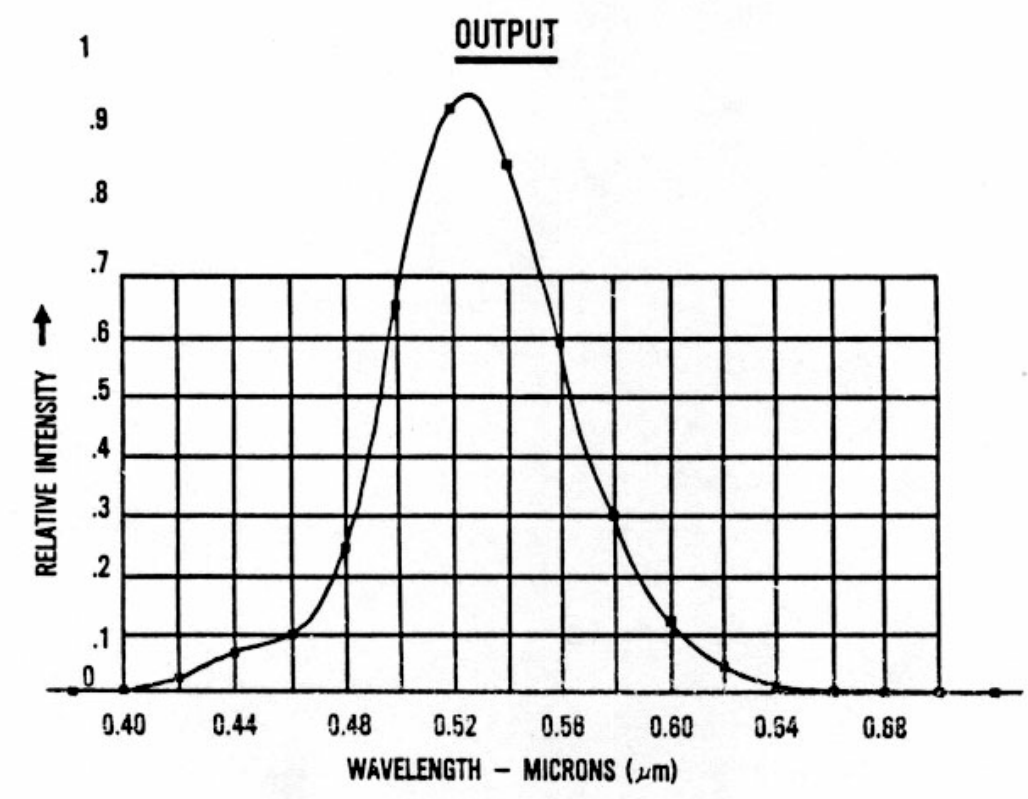

Fig. 11. Spectral output of GEN II and GEN III image intensifier.

Table 1 compares some of the performance parameters for second and third generation image intensifiers.

Table 1. Comparison on Second Generation (GEN II) and Third Generation (GEN III) Image Intensifiers

\begin{tabular}{|l|c|c|}
\hline & Second Generation & Third Generation \\
\hline System Gain & 400 & 2000 \\
\hline Resolution & $24 \mathrm{lp} / \mathrm{mm}$ & $36 \mathrm{lp} / \mathrm{mm}$ \\
\hline f/number & $\mathrm{f} / 1.4$ & $\mathrm{f} / 1.2$ \\
\hline Photocathode Material & $\mathrm{S}-20$ & $\mathrm{GaAs}$ \\
\hline $\begin{array}{c}\text { Photocathode Response } \\
\mathbf{8 3 0} \mathbf{~ n m} \\
\mathbf{8 8 0} \mathbf{~ n m}\end{array}$ & $15 \mathrm{~mA} / \mathrm{W}$ & \\
\hline Output (530 nm) & $0.5 \mathrm{~mL}$ & $100 \mathrm{~mA} / \mathrm{W}$ \\
\hline Battery Life & $10 \mathrm{hours}$ & $600 \mathrm{~mA} / \mathrm{W}$ \\
\hline
\end{tabular}




\begin{tabular}{|l|c|c|}
\hline Weight & $1.9 \mathrm{lb}$ & $1.0 \mathrm{lb}$ \\
\hline
\end{tabular}

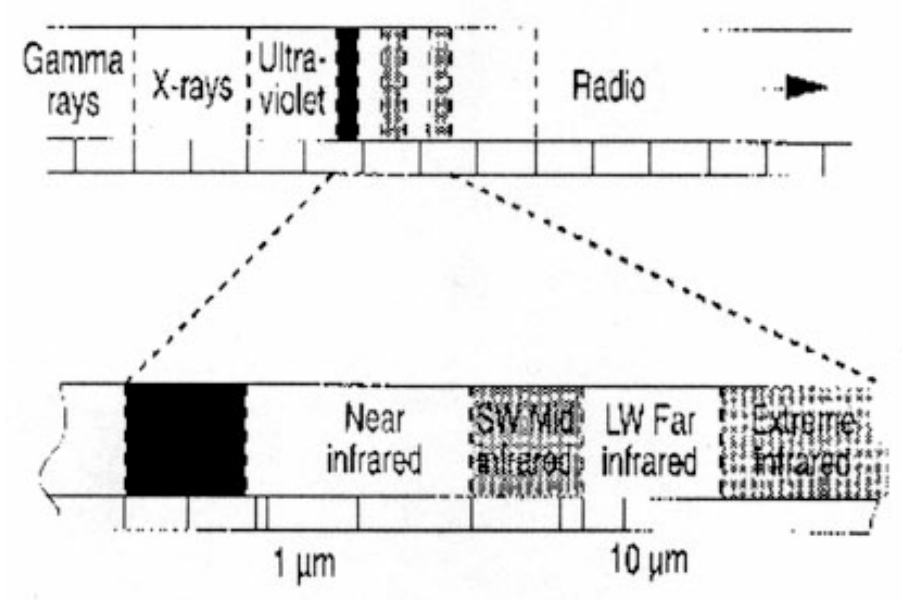

Fig. 12. The electromagnetic spectrum.

\begin{abstract}
Emissivity
Infrared imagers measure and image the emitted infrared radiation from an object. The fact that radiation is a function of object surface temperature makes it possible for the camera to calculate and display these temperatures. However, the radiation measured by the camera does not only depend on the objects temperature, but is also a function of the object's emissivity. Emissivity is a measure of how much radiation is emitted from the object compared to that if it was a perfect blackbody. A blackbody is an object which absorbs all radiation that impinges on it at any wavelength. The apparent misnomer "black" relating to an object emitting radiation is explained by Kirchhoff's Law, which states that a body capable of absorbing all radiation at any wavelength is equally capable of emitting radiation at any wavelength. Emissivity is the ratio of the amount of energy radiated from a body of interest divided by the amount of energy radiated from a blackbody. It is always 1 (for a blackbody) or less.
\end{abstract}

Normally, materials and surface treatments exhibit emissivities ranging from 0.1 to 0.95 . A highly polished (mirror) surface falls below 0.1 , while an oxidized or painted surface has much higher emissivity. Oil-based paint, regardless of color in the visible spectrum, has an emissivity over 0.9 in the infrared. Human skin exhibits an emissivity close to 1. Non-oxidized metals represent an extreme case of almost perfect opacity and high specular reflectivity, which does not vary greatly with wavelength. Consequently, the emissivity of metals is low-only increasing with temperature. For non-metals, emissivity tends to be high, and decreases with temperature. Typical emissivities for a variety of common materials are listed in Table 2.

\title{
Brightness and Contrast
}

With infrared imagers, the terms "brightness" and "contrast" are used to describe the appearance of the thermal image. In this case, brightness actually refers to the heat radiation level at which the thermal image is set for producing a good image on the screen. A typical thermal imager is able to resolve thousands of levels of heat radiation and display them as color or grey scales on a monitor. Displaying the whole range of thermal levels creates an image with little thermal contrast. Contrast, on the other 
hand, is defined as the ratio between the maximum and minimum brightness values in the display. In this case, contrast actually refers to the range of heat levels provided by a particular thermal image, and is often defined as "gain" or "span." The contrast can be increased by choosing to display a small range of thermal levels, called the span. The narrower the span, the higher the contrast. When the span and level have been set, the thermal radiation below the span appears black, and the radiation above appears white. 


\section{Sensitivity and Resolution}

Two of the most important parameters to consider in an infrared imager are sensitivity and resolution. Sensitivity is a measure of the minimum temperature difference which can be detected or viewed. A system with high sensitivity is able to detect very small temperature differences. Resolution, sometimes called geometrical or spatial resolution, is a measure of the minimum size which can be resolved by a system. A system with high resolution can detect very small objects.

In order to create an infrared image of an object, a very small infrared sensitive detector cell placed in the focal plane of the imager is required to sense the radiation from one point on the object. Since the detector cell "converts" infrared radiation into an electronic signal, this signal can be processed by the camera to provide information on the temperature of the object at that point. The smaller the cell size chosen, the smaller the area of the object which is being sensed. This means that small detector cells must be used to create a thermal imaging system with high spatial resolution. Since, the signal level produced by a cell is proportional to its area, this also means that high spatial resolution is achieved at the expense of signal strength (and hence sensitivity).

\section{Integration Time}

Signal strength is improved by using longer integration times. The best integration times are obtained by using a matrix of detector cells to receive radiation from an object. Since one cell senses one part of the object all the time, integration times in the order of 15 to 20 milliseconds can be achieved. With such a high integration time, it is possible to decrease the cell size (and hence increase resolution) without impairing signal quality too much. Thermal imagers which use an array of detectors in the focal plane in the thermal imaging system are therefore able to achieve high levels of image resolution.

\section{Cooled Infrared Imagers}

Infrared detectors are classified as either photon detectors or thermal detectors. Photon detectors, usually photoconductors or photodiodes produce an electrical response directly as the result of absorbing IR radiation. These detectors can be very sensitive, but noise producing mechanisms depend strongly on temperature. It is necessary to cryogenically cool these detectors to liquid nitrogen temperatures $\left(77^{\circ} \mathrm{K}\right.$, or $-196^{\circ}$, or $-320^{\circ} \mathrm{F}$ ) in order to maintain high sensitivity.

\section{Stirling Cycle Coolers}

In order to keep the infrared detector at cryogenic temperatures, the detector is integrated into a miniature dewar/cooler/detector assembly. This assembly eliminates the need to actually cool with liquid nitrogen. The closed (Stirling) cycle microcooler in this assembly will bring the detector down to operating temperature in 5 to 8 minutes and requires only about 3 watts of power. The Stirling-cycle microcooler is fundamentally a compression-expansion refrigerator with no valves; instead, it incorporates a regenerator. The regenerator is a tube of porous material that has low thermal conductivity to maintain a temperature gradient and high heat capacity to act as an efficient heat exchanger. The regenerator is located between a reciprocating piston and the cold well and moves back and forth in synchronization with the piston.

Helium gas is used as the working fluid. The microcooler cycle cools a few degrees at a time; the cold well, regenerator, and detector are among the parts that must be cooled. Eventually the desired temperature is reached at the detector. The system senses this and throttles back the cooler motor just enough to maintain the desired temperature. A schematic of an example microcooler assembly is shown in Figure 13 and a photograph of a commercial microcooler (FLIR Systems) is shown in Figure 14. 
Table 2. Emissivity of Common Materials

\begin{tabular}{|c|c|c|c|}
\hline Metals and Oxides & & Temp C & Emissivity \\
\hline \multirow[t]{2}{*}{ Aluminum: } & foil (bright) & 20 & 0.04 \\
\hline & Weathered & 20 & $0.83-0.94$ \\
\hline \multirow[t]{2}{*}{ Copper: } & Polished & 100 & 0.05 \\
\hline & heavily oxidized & 20 & 0.78 \\
\hline \multirow[t]{2}{*}{ Iron: } & cast, oxidized & 100 & 0.64 \\
\hline & sheet, heavily rusted & 20 & $0.69-0.96$ \\
\hline Nickel: & electroplated, polished & 20 & 0.05 \\
\hline \multirow[t]{2}{*}{ Stainless Steel: } & Polished & 20 & 0.16 \\
\hline & Oxidized & 60 & 0.85 \\
\hline \multirow[t]{2}{*}{ Steel: } & Polished & 100 & 0.07 \\
\hline & Oxidized & 200 & 0.79 \\
\hline Brick: & common red & 20 & 0.93 \\
\hline Carbon Candle Soot: & & 20 & 0.95 \\
\hline Concrete: & Dry & 35 & 0.95 \\
\hline Glass: & chemical ware & 35 & 0.97 \\
\hline \multirow[t]{4}{*}{ Oil: } & Lubricating & 17 & 0.87 \\
\hline & film thickness $0.03 \mathrm{~mm}$ & 20 & 0.27 \\
\hline & film thickness $0.13 \mathrm{~mm}$ & 20 & 0.72 \\
\hline & thick coating & 20 & 0.82 \\
\hline Paint, oil: & average of 16 colors & 20 & 0.94 \\
\hline Paper: & White & 20 & $0.07-0.90$ \\
\hline Plaster: & & 20 & $0.86-0.90$ \\
\hline Rubber: & Black & 20 & 0.95 \\
\hline Skin: & Human & 32 & 0.98 \\
\hline \multirow[t]{2}{*}{ Soil: } & Dry & 20 & 0.92 \\
\hline & saturated with water & 20 & 0.95 \\
\hline \multirow[t]{3}{*}{ Water: } & Distilled & 20 & 0.96 \\
\hline & frost crystals & -10 & 0.98 \\
\hline & Snow & -10 & 0.85 \\
\hline Wood: & planed oak & & 0.90 \\
\hline
\end{tabular}




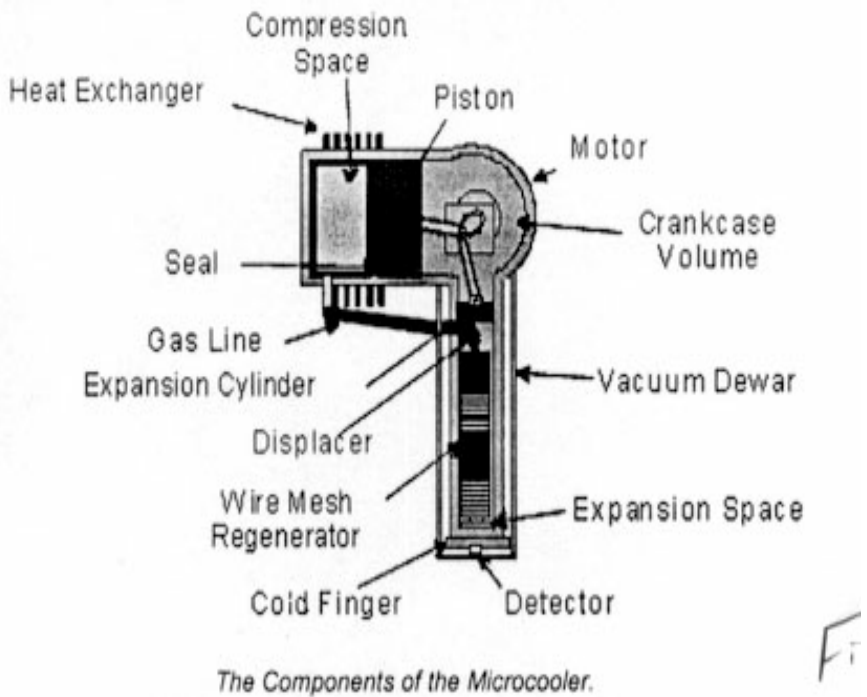

Fig. 13. The components of the microcooler.

Focal Plane Array Detectors

The early infrared imagers required that a limited number of detector elements be "scanned" over the scene of interest in order to produce an infrared image of the scene. Because of sensitivity, resolution, and integration time issues, most modern infrared imagers use focal plane arrays (FPA) for the sensing elements. These arrays are typically 256 X 256 elements and are referred to as "starring" arrays because they continually look at the scene of interest or "stare" at the scene. A typical focal plane array material for these cooled infrared imagers is platinum silicide (PtSi) which is sensitive to infrared wavelength in the 3 to $5 \mathrm{~nm}$ range.

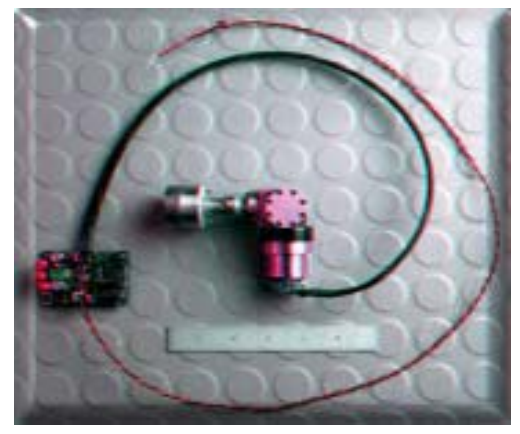

Fig. 14. Commercial microcooler/detector assembly.

A platinum silicide detector works like a photodiode, that is when infrared light of the proper wavelength ( 3 to $5 \mathrm{~nm}$ ) strikes each individual detector, the detector will change its resistance to a bias current imposed on it and allow and increase in current flow. The amount of current is proportional to the amount of infrared energy striking the detector. The electric charge carrier must cross a Shottky barrier 
between the PtSi and the silicon in order to lower the resistance and increase the current flow. Many electric charge carriers cannot make it through the barrier because they do not have enough energy. This limits the efficiency with which this type of detector can convert IR photons to measurable current. Because the individual array elements (detectors) are always staring at a section of the scene more than offsets this and provides excellent sensitivity. Such a focal plane array can resolve temperature differences of less than $0.1^{\circ} \mathrm{C}$. A graphical example of how the PtSi FPA works is shown in Figure 15.

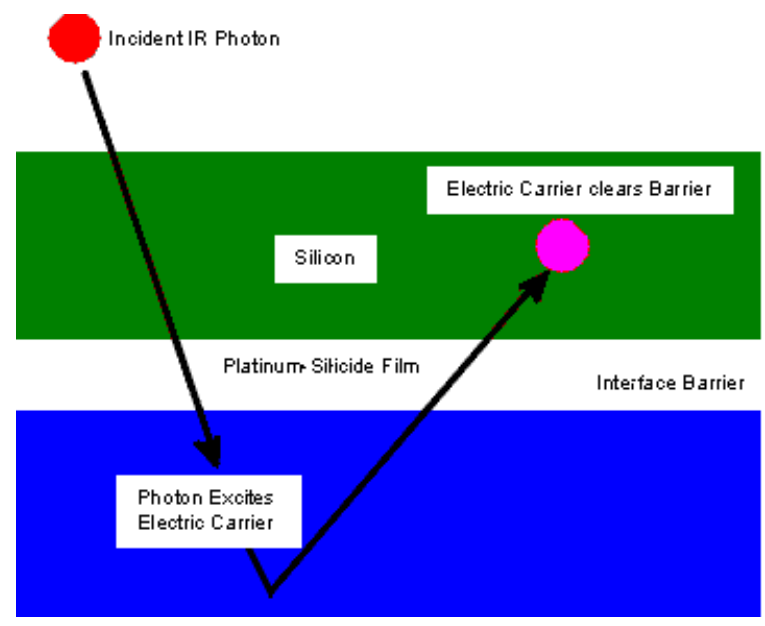

Fig. 15. Graphical description of photodiode infrared detector

\section{Uncooled Infrared Imagers}

Thermal detectors experience a temperature change when they absorb IR radiation, and an electrical response results from the temperature dependence of some material property. These detectors are thermally isolated from their immediate surrounding to maximize the temperature change that results from the absorption of a small amount of IR radiation. Thermal detectors are not generally as sensitive as photon detectors, but they retain a good level of performance at room temperature. This enables the fabrication of infrared imagers that do not require cryogenic cooling.

An uncooled infrared imager system has many advantages over conventional cooled infrared systems. Since it is simpler, lacking the complexity of a cryogenic cooler, the uncooled system will be smaller, lighter, lower power, and less costly than its cooled counterparts. It will be more reliable, because it does not contain a complex mechanism such as a cryogenic cooler. The uncooled detector operates near roomtemperature, or $295^{\circ} \mathrm{K}$. A single-stage thermoelectric cooler in the detector package, maintains the roomtemperature operation of the detector over varying ambient temperatures.

\section{Ferroelectric Materials}

Typically, uncooled focal plane arrays have fewer elements than cooled focal plane arrays; $128 \mathrm{X} 128$ elements for uncooled arrays versus 256 X 256 for cooled arrays. Uncooled focal plane arrays are based on ferroelectric (or alternatively pyroelectric) materials. Just as a ferromagnetic material retains magnetic polarization after application and removal of a magnetic field, so does a ferroelectric material retain electric polarization after application and removal of an electric field. This remnant polarization depends on temperature. The slope of the polarization verus temperature curve is the pyroelectric coefficient. The pyroelectric coefficient peaks near the transition temperature, the temperature above which the material ceases to be ferroelectric. A capacitor made from such a material can be used as an IR detector, and the 
pyroelectric coefficient is a measure of its responsivity. Operation near the responsivity peak (transition temperature) would normally be impractical because of instability associated with the transition.

However, the application of an electric field solves this problem by stabilizing the polarization and further improves responsivity.

An alternative method of operating these materials, know as the dielectric bolometer mode, provides useful performance above the transition temperature which is convenient for those materials where the transition temperature is close to ambient. In this case, the material is operated in an applied electrical field and a pyroelectric effect is induced. In this mode, the dielectric constant of the material is a strong function of temperature, and the device can effectively be considered as a capacitor storing an applied voltage. Changes in material temperature cause the capacitance to change, and by conservation of charge, the voltage across the capacitor changes from the stored reference, and this can be read as a signal by an external amplifier. Thus, in dielectric bolometer mode, the device must be "reset" periodically to a reference voltage. This is in contrast to other ferroelectric material based sensors which must use "choppers" to provide a temperature contrast for the sensor.

\section{Choppers}

A useful thermal imaging system must be able to resolve objects that differ in temperature from the environment by much less than $1^{\circ} \mathrm{C}$. The difference in the amount of IR radiation emitted by two objects that differ in temperature by $1{ }^{\circ} \mathrm{C}$ is a tiny fraction of the total radiation emitted by each. To minimize the dynamic range requirements for the processing electronics, it is desirable to remove the average background component and amplify only the temperature differences. This can be implemented by a mechanical chopper which provides time modulation of the scene.

The uncooled detector elements act as series capacitors into the electronics and require the IR energy to be modulated. This modulation is performed by a chopper in the uncooled system. The detector will generate a signal that represents the difference in temperature between the apparent chopper temperature and the scene temperature. Since the detector requires each row of pixels to be read out sequentially, uncooled systems use a rotating disc with a special pattern called an Archimedes' spiral. This pattern emulates an edge moving across the rows of the detector synchronized with electronic sampling. Different implementations fall into two categories, opaque or diffusing. The opaque chopper is made of thin metal or plastic and has an open spiral pattern. The detector views the scene through the cutout and views the chopper itself during the closed mode. This type of chopper disc shown in Figure 16 is the most desired, because of its simplicity, low cost, and production possibilities. In a defocusing or diffusing chopper, the disc is made of a material that transmits the IR energy such as germanium or silicon. Features build into the spiral pattern defocus the image, which produces a temperature reference that resembles the scene average. By using a reference temperature that closely resembles the actual scene, the dynamic range 


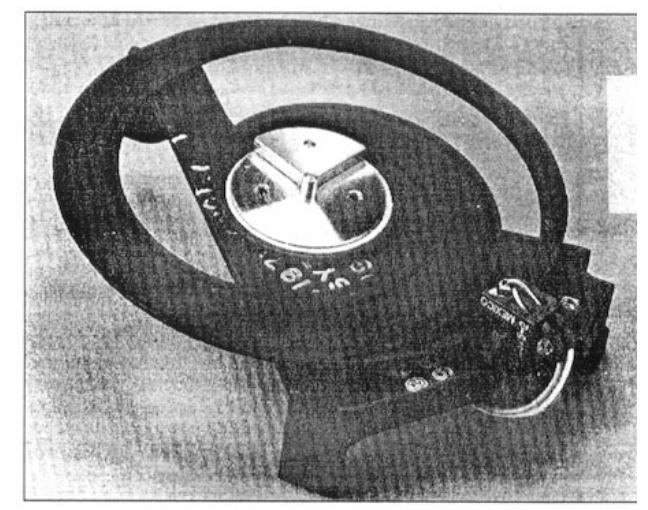

Fig. 16. Opaque chopper assembly.

requirements are greatly reduced. Both choppers are driven with a DC motor and include a position sensor, to synchronize the chopper with the electronics.

\section{Optics}

For an uncooled infrared imaging system, the range and resolution are limited by the size, spacing, and number of pixels in the array as shown in Figure 17, as well as a lower sensitivity to temperature differences. Uncooled systems therefore require larger, faster optics to achieve comparable performance capabilities. Uncooled sensors have used a variety of IR lens assemblies. The lens on a sensor determines its Field of View (FOV), resolution, range, and sensitivity for a given detector. The sensitivity is controlled by the lens f-number. The faster the lens, the more energy is collected at the detector surface; and therefore, the more sensitive the system. The FOV, resolution and range are interrelated parameters determined by the lens focal length and configuration, as well as the size and number of detector pixels. The lens can be either refractive or reflective.

In refractive optics, the IR energy transmits through the lens elements, usually made of germanium, to be focused onto the IR energy onto the detector. These reflectors can be any stable material, such as plastic or metal, as long as the mirrors are highly reflective to the desired wavelengths of energy. Military systems typically use refractive optics for the performance, weight, and size advantages, while commercial systems have used the potentially lower cost reflective optics with reasonable performance.

\section{Hand-Held Versus Vehicle Mounted Imagers}

There are generally two classes of infrared imagers: handheld and vehicle mounted. The handheld imagers are typically, lighter, smaller, and less capable than vehicle (usually aircraft) mounted systems. Vehicle mounted systems usually utilize focal plane arrays with many more elements (1024 X 1024) than hand-held systems which gives them greater resolution. 


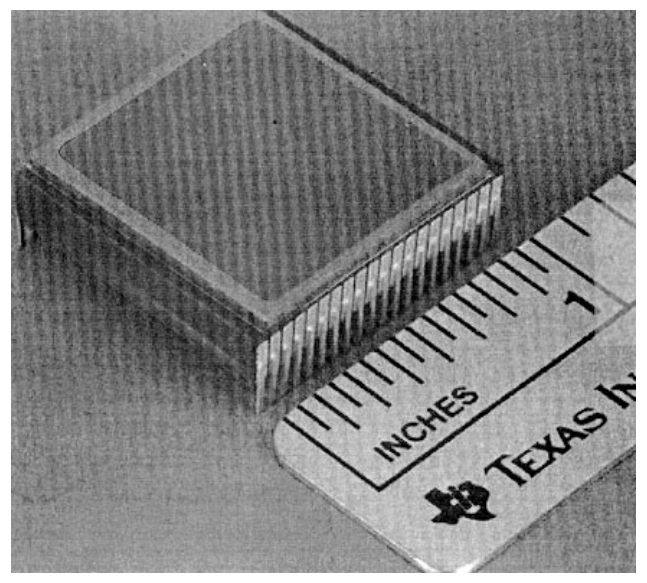

Fig. 17. Uncooled infrared detector.

\section{Factors Influencing Detection with Night Vision Devices}

As previously stated, night vision devices do not truly turn night into day, but they do enhance vision in low light level scenarios. Image intensifiers and infrared imagers operate in different spectral regions of the electromagnetic spectrum using different physical principals, materials, and electronic technology. As a result, the ability to detect and identify an object by each type of night vision device is affected by different parameters. This section will identify the parameters which affect each type of night vision device and provide an understanding of the effects of these parameters on the devices. 


\section{Common Factors}

Table 3 illustrates many of the factors which influence detection and imaging of night vision devices.

\section{Atmospheric Constituent Absorption}

To understand the effect of the atmosphere on night vision detection, first consider the amount of illumination (electromagnetic radiation) which is incident upon the earth. Figure 18 illustrates the levels of incident illumination as a function of solar and lunar altitude and various phases of the moon. The spectral distribution of this radiation is also important for night vision devices, especially image intensifiers, which can only amplify the light which is present. Figure 19 presents the spectrum of the night sky light (moonlight or starlight) for various phases of the moon. Figure 20 details the spatial radiance differences in full moon, quarter moon, and starlight in the $450 \mu \mathrm{m}$ band which covers the spectral response bands at both GEN II and GEN III image intensifiers. As previously stated, atmospheric constituents absorb portions of the incident radiation from sunlight, moonlight, or starlight. Figure 21 presents the levels of incident electromagnetic radiation on the earth during daylight and illustrates the effect of absorption by atmospheric constituents on the electromagnetic spectrum. These absorption effects occur during both day and night. Figure 22 shows the spectral absorption of selection atmospheric constituents and the resultant daylight spectrum. In comparison to Figure 21, Figure 22 plots transmittance instead of intensity level. Figure 23 presents a typical atmospheric transmittance spectrum. This atmospheric transmittance is affected by geometry of the optical path with respect to the surface terrain and the surroundings, air temperature, atmospheric pressure, relative humidity, number and size distribution of atmospheric aerosols, concentration of abnormal atmospheric constituents in the optical path such as smoke, dust, exhaust fumes, and chemical effluents, and refractive indices of all types of aerosol in the optical path. Figure 24 presents a simplified atmospheric transmittance spectrum illustrating the carbon dioxide $\left(\mathrm{CO}_{2}\right)$ and water $\left(\mathrm{H}_{2} \mathrm{O}\right)$ absorption bands. Figures 25 and 26 present the computed spectra of atmospheric transmittance for five model atmospheres for surface visibility of $23 \mathrm{~km}$ and $5 \mathrm{~km}$, respectively. These five model atmospheres represent typical areas of the world; tropical summer, mid-latitude summer, subarctic summer, mid-latitude winter, and subarctic winter. From these figures, it can be seen that the greatest variability in transmittance occurs in the 8-12 $\mu \mathrm{m}$ spectral region.

\section{Fog and Haze}

For Naval Special Operations, fog and haze can be important considerations in determining ranges of detectability. There has been a study (Vatsia, 1972) $)^{3}$ of the transmittance of the electromagnetic radiation "atmospheric window" through artificial fog. This artificial fog simulates the important aspects of "light" transmittance through real fog. This study measured the relative transmittance in the "visible", 3-5 $\mu \mathrm{m}$, and $8-12 \mu \mathrm{m}$ spectral bands through a 150 foot optical path. Figure 27 presents the relative transmittance for the visible 3-5 $\mu \mathrm{m}$, and 8-12 $\mu \mathrm{m}$ spectral band imagers through artificial fog of varying optical density or transmittance. From this figure, it is clear that the relative spectral band transmittance through fog increases with wavelength in the three atmospheric windows under consideration. However, the transmittance for the 3-5 $\mu \mathrm{m}$ and 8-12 $\mu \mathrm{m}$ spectral band falls to 0.02 and 0.2 respectively, when the optical density of fog reaches a value of 2 (visible transmission of 0.01.) Further, the relative transmittance of the 8-12 $\mu \mathrm{m}$ spectral band imagers falls below a rather low value of 0.02 when the optical density of fog reaches 2.7 (visible transmittance of 0.002 ). The real outdoor fogs have optical densities varying from 1.7 to 30 which correspond to visible ranges of $1 \mathrm{~km}$ to 50 meters, respectively. The $8-12 \mu \mathrm{m}$ spectral band imager has a reasonable transmittance advantage over the visible and the $3-5 \mu \mathrm{m}$ band imagers specially in the optical density range of 1.7 to 2.7 (visible range of 1000 to $630 \mathrm{~m}$ ) where the transmittance of the 3-5 $\mu \mathrm{m}$ imagers falls below 0.02 . Figure 27 illustrates the relationship between visible range in fogs, fog density, transmittance, and wavelength for various fogs. Figure 28 shows similar information for haze. 
Table 3. Factors Affecting Detection and Imaging

\begin{tabular}{|c|c|}
\hline \multirow[t]{4}{*}{ Atmospheric Transmission: } & Molecular Absorption and Scattering \\
\hline & Water Vapor and $\mathrm{CO}_{2}$ \\
\hline & Aerosol Extinction \\
\hline & Precipitation \\
\hline \multirow[t]{2}{*}{ Path Conditions: } & Path Radiance (Scattered or Emitted) \\
\hline & Turbulence \\
\hline \multirow[t]{5}{*}{ Scene Characteristics: } & Intrinsic Contrast \\
\hline & Clutter \\
\hline & Target Angular Size and Range \\
\hline & Target/Background Spectral Content \\
\hline & Target Motion \\
\hline \multirow[t]{4}{*}{ Imaging System Characteristics: } & Wide/Narrow Field of View \\
\hline & Detector Sensitivity \\
\hline & Signal/Noise (Detection) Threshold \\
\hline & Signal Processing and Display \\
\hline \multirow[t]{3}{*}{ Human Characteristics: } & Contrast Threshold \\
\hline & Variability Among Operators \\
\hline & Variability Within an Operator \\
\hline
\end{tabular}

For infrared sensors, temperature signals "seen" by the sensor result from temperature values and variations that exist at the target, attenuated by the atmosphere intervening between the sensor and the target. The target-to-background contrast (degrees kelvin) is a temperature difference (target temp background temp), not a ratio or percentage as are typically used to describe contrast of objects and represent typical areas of the world; tropical summer, mid-latitude summer, subarctic summer, midlatitude winder, and subarctic winter. From these figures, it can be seen that the greatest variability in transmittance occurs in the $8-12 \mu \mathrm{m}$ spectral region. 


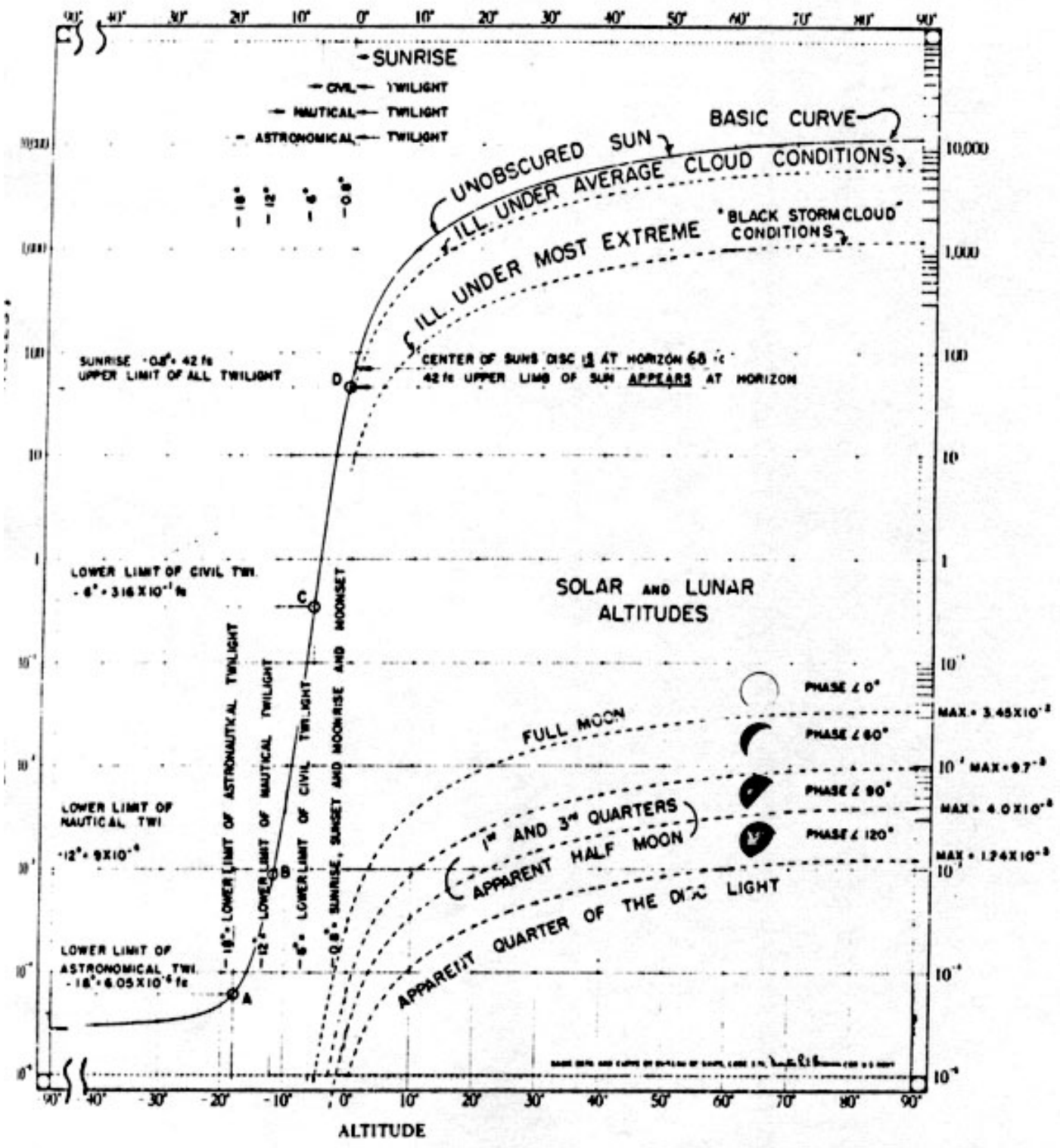

Fig. 18. Natural luminous incidence (illuminance) as a function of solar and lunar altitude and for various phases of the moon as measured by Brown. 


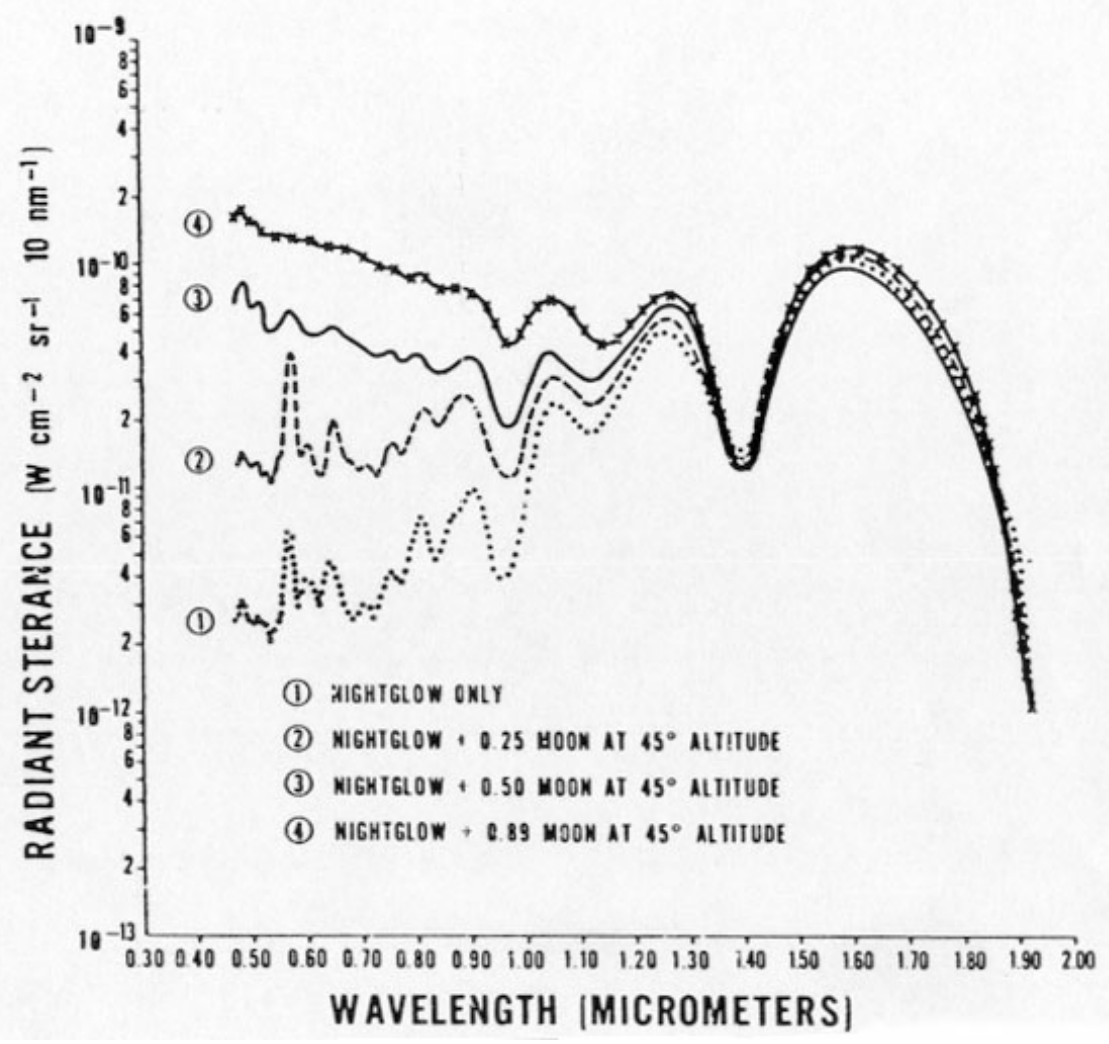

Fig. 19. Night sky spectral radiant sterance for various phase of the moon.

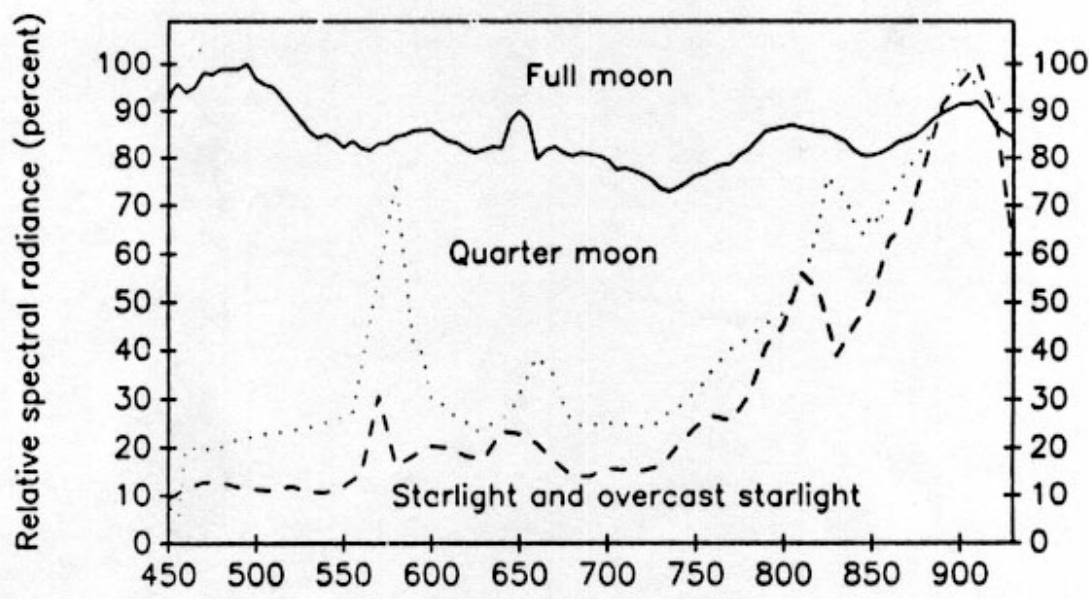

Fig. 20. Detailed night sky radiance in the $450 \mu \mathrm{m}$ to $900 \mu \mathrm{m}$ band.

represent typical areas of the world; tropical summer, mid-latitude summer, subarctic summer, midlatitude winder, and subarctic winter. From these figures, it can be seen that the greatest variability in transmittance occurs in the $8-12 \mu \mathrm{m}$ spectral region. 
background temp), not a ratio or percentage as are typically used to describe contrast of objects and bacground in the visible spectrum. This temperature difference, modulated by the atmospheric transmittance, is usually referred to as "delta T" at the sensor. The chemical constituents of the atmosphere between the sensor and the target affect the temperature contrast at the sensor by scattering and absorbing the thermal radiation emitted from the target and the background. The scattering and absorption mechanisms are wavelength dependent functions, and thus the wavelengths of the thermal radiation and the wavelength sensitivity of the sensor are factors in determining the temperature contrast at the sensor. The uncooled infrared sensors use detector materials that are sensitive in the 8 to $12 \mu \mathrm{m}$ wavelength region of the electromagnetic spectrum. In this relatively long wavelength region, the targetto-background contrast is almost entirely affected by atmospheric absorption. Figure 29 illustrates atmospheric transmission in the 8-12 $\mu \mathrm{m}$ range as a function of range for weather conditions titled "Best", "Medium", and "Worst". Generally, "Best" means good visibility, low relative humidity, and rural aerosols. "Medium" means average visibility, moderate relative humidity, and urban aerosols. "Worst" means poor visibility, high relative humidity, and adverse fog aerosol. After much investigation, a study (Berry, Perez, Houchard, Stellini, and Sanchez, 1994) ${ }^{4}$ concluded that in the 8 to $12 \mu \mathrm{m}$ band, a linear relationship between atmospheric transmission and perceptible water vapor in the atmosphere between the sensor and the target is a good approximation when the sensor and the target are near sea level altitude. Figure 30 illustrates the atmospheric transmittance in the 8 to $12 \mu \mathrm{m}$ region as a function of humidity. These figures illustrate that the atmosphere model does not have to be sophisticated, and that the sensor does not have to be very far from the target to have atmospheric transmission of less than 10 percent, if the weather conditions are between poor and average. Generally, the weather conditions are such for about one-half of the time regardless of where one is located.

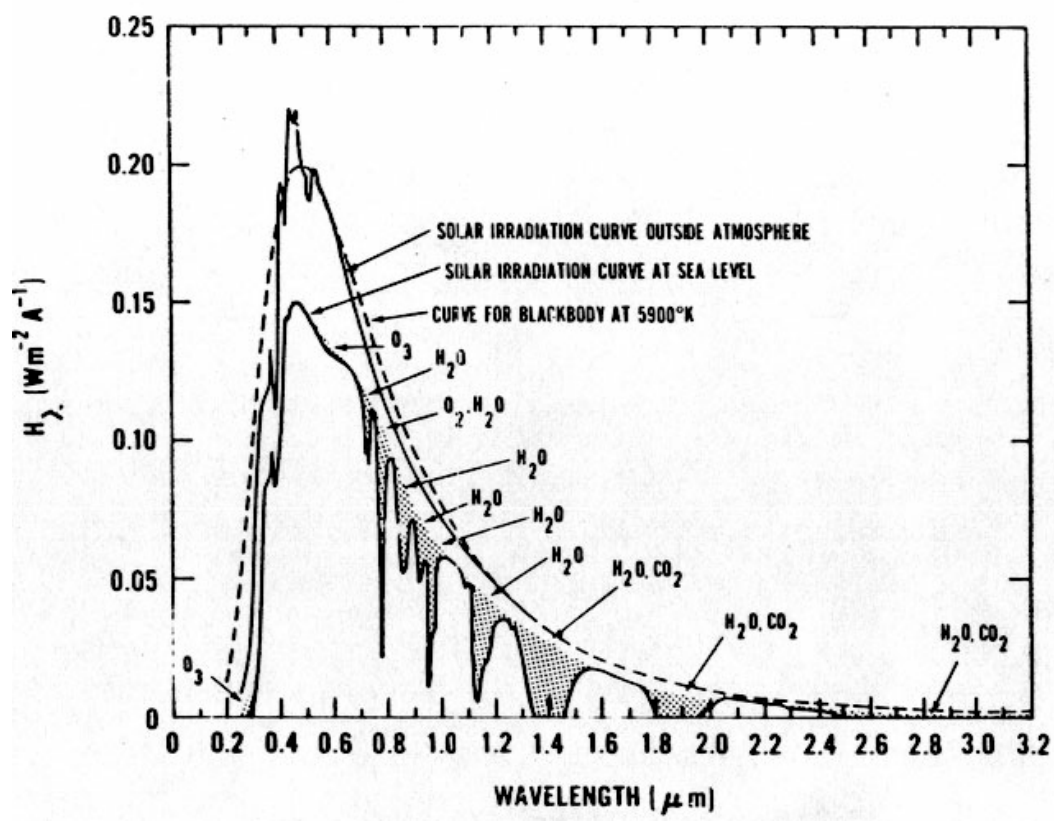


Fig. 21. The solar spectral radiant incidence (irradiance) at sea level for one air absorption by atmospheric constituents is indicated by shaded areas.

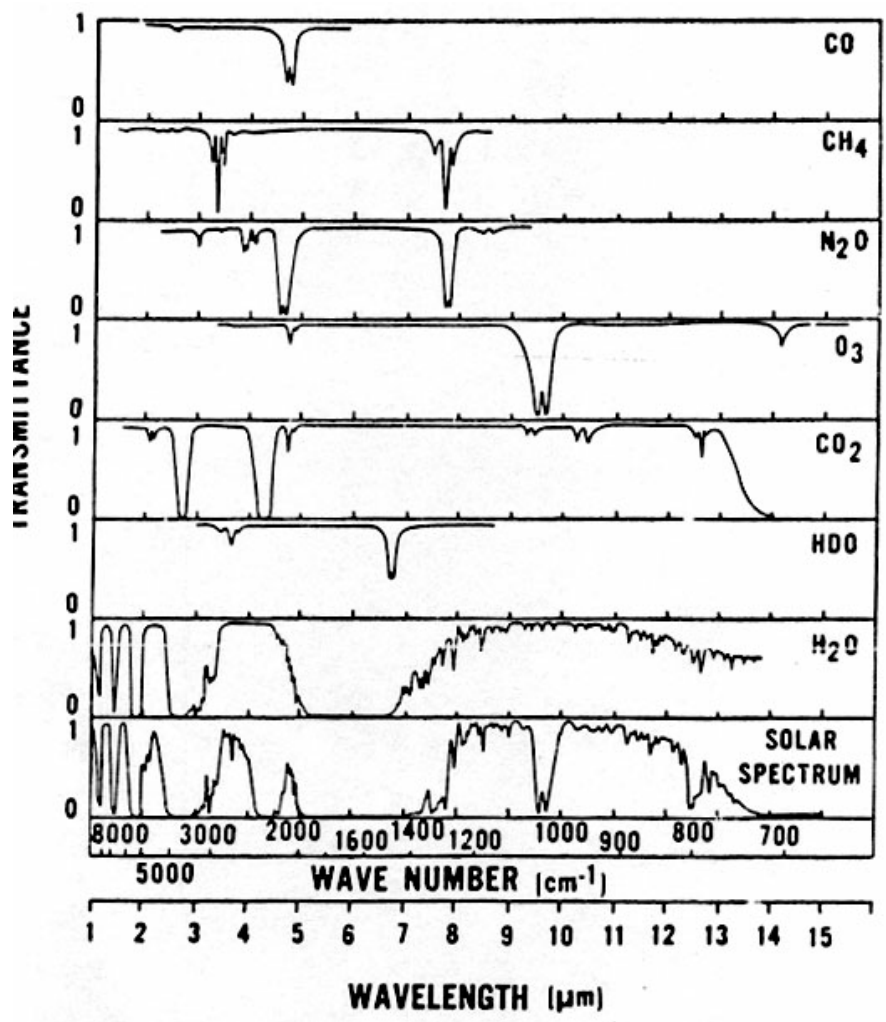

Fig. 22. Spectral transmittance of atmospheric gases and the solar radiant incidence spectrum. 


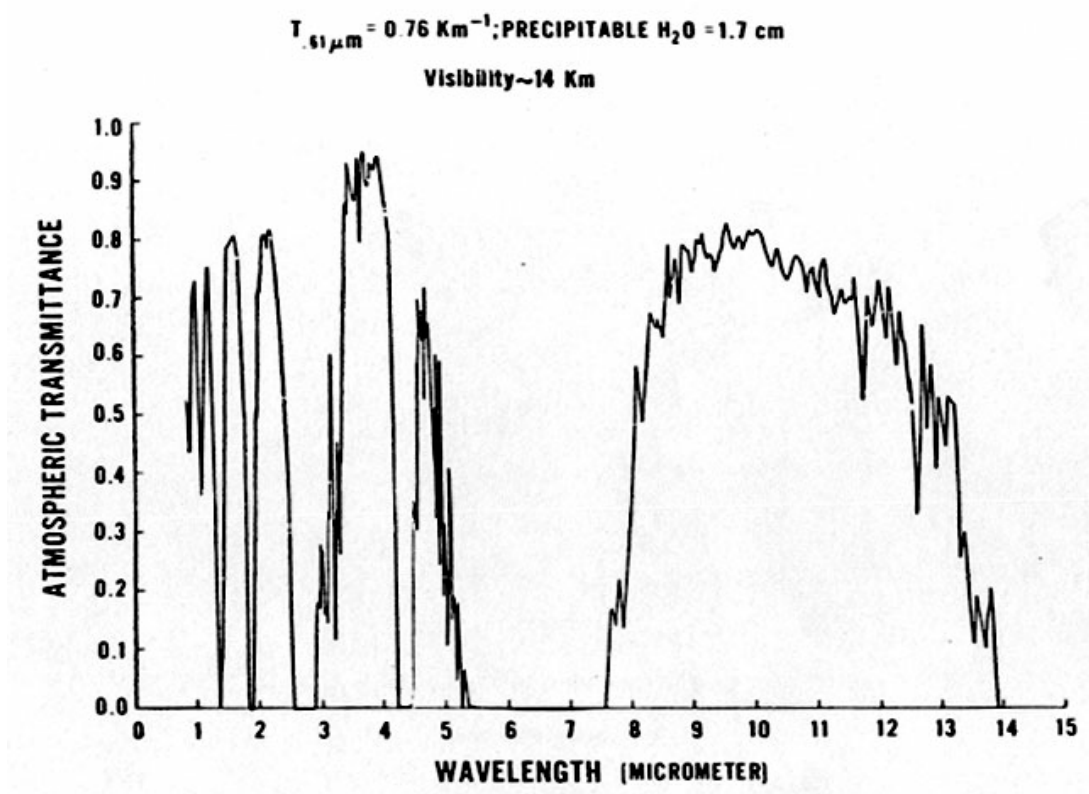

Fig. 23. Typical atmospheric transmittance spectrum.

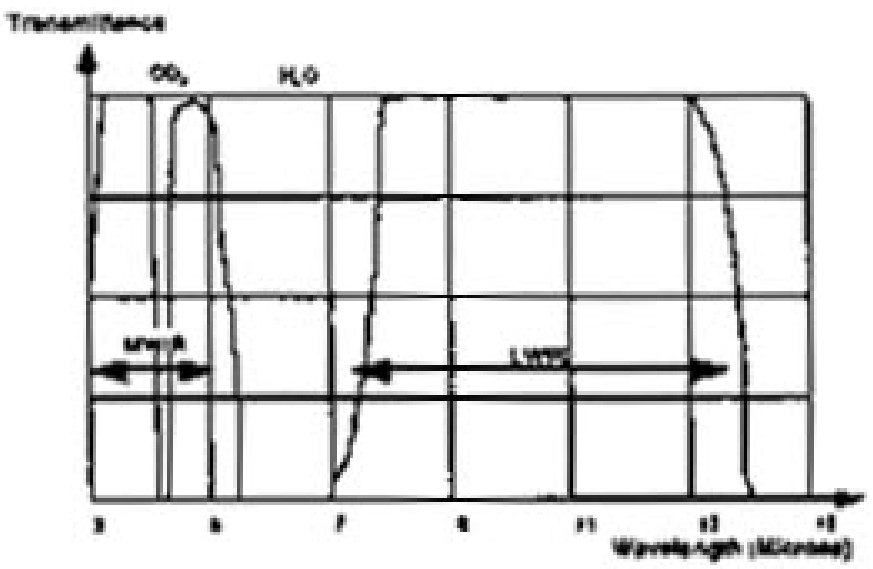

Fig. 24 Atmospheric transmittance spectrum. carbon dioxide $\left(\mathrm{CO}_{2}\right)$ and water absorption bands.

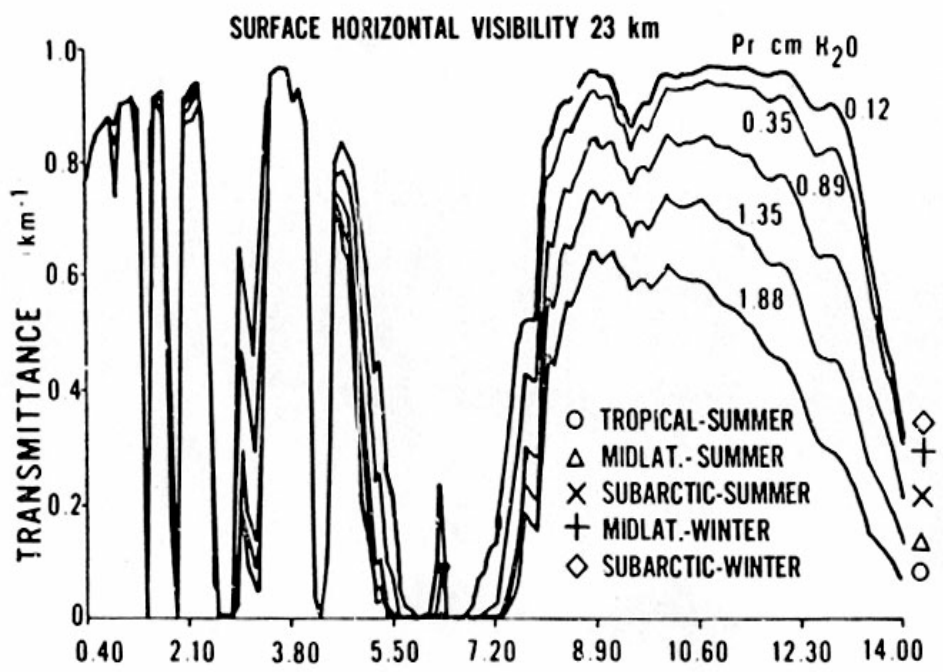

Shows

$\left(\mathrm{H}_{2} \mathrm{O}\right)$ 
Fig. 25. Computed spectra of atmospheric transmittance for five model atmospheres with $23 \mathrm{~km}$ horizontal visibility.

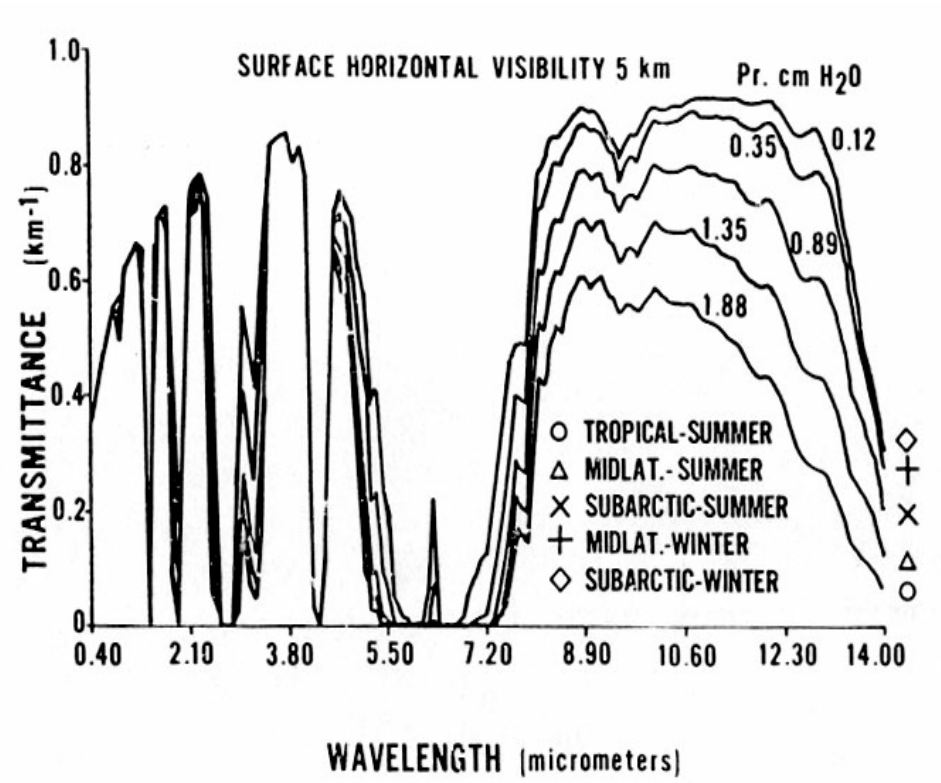

Fig. 26. Computed spectra of atmospheric transmittance for five model atmospheres with $5 \mathrm{~km}$ horizontal visibility 
Fig. 27. Atmospheric selective fogs.

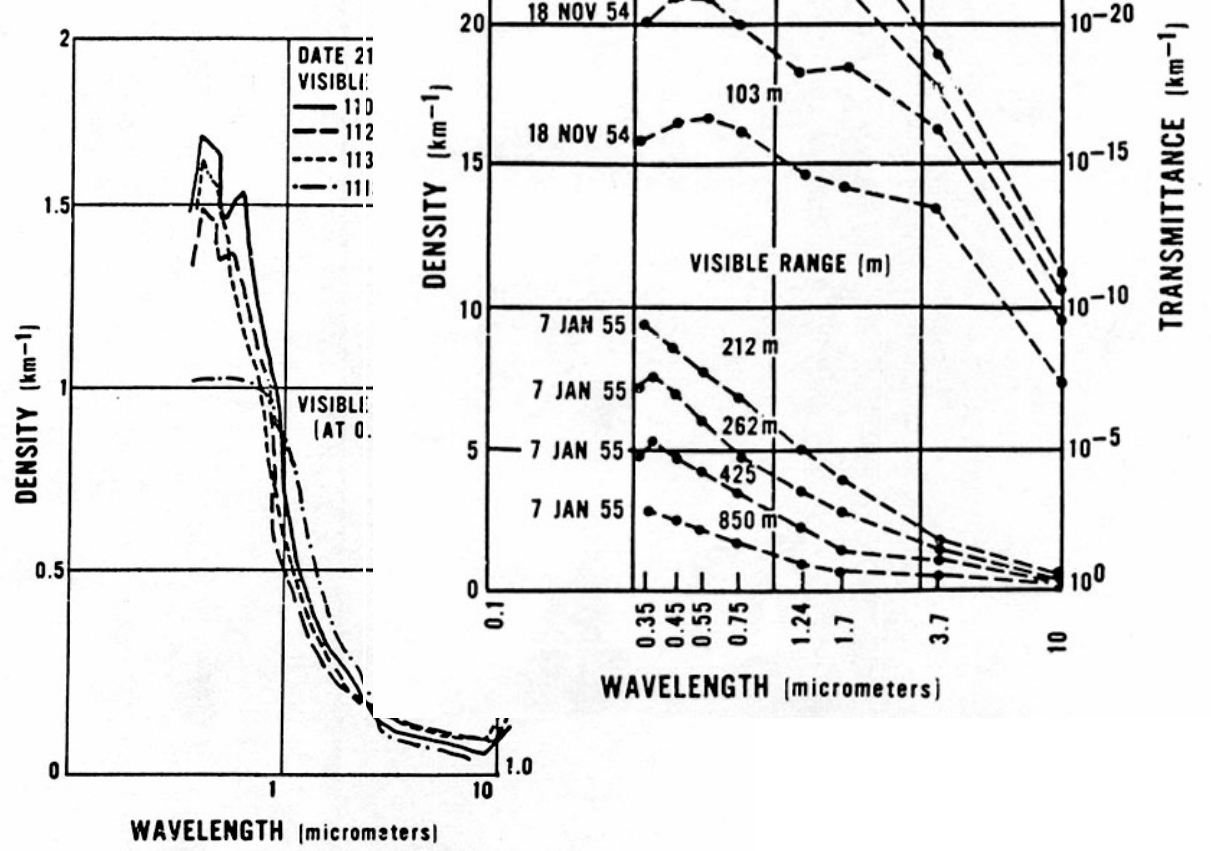

transmittance spectra for

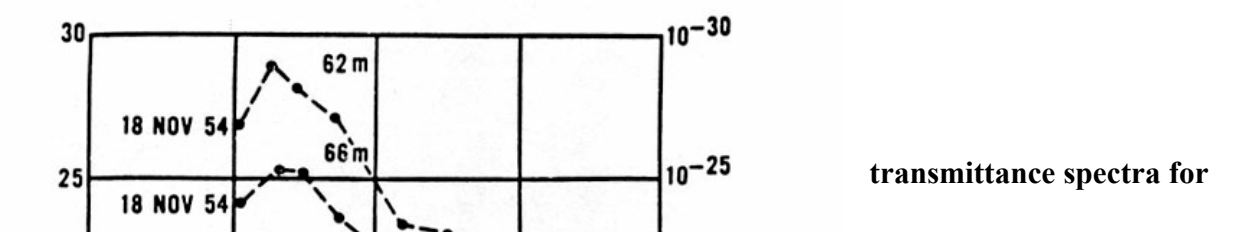


Fig. 28. Atmospheric transmittance spectra for haze.

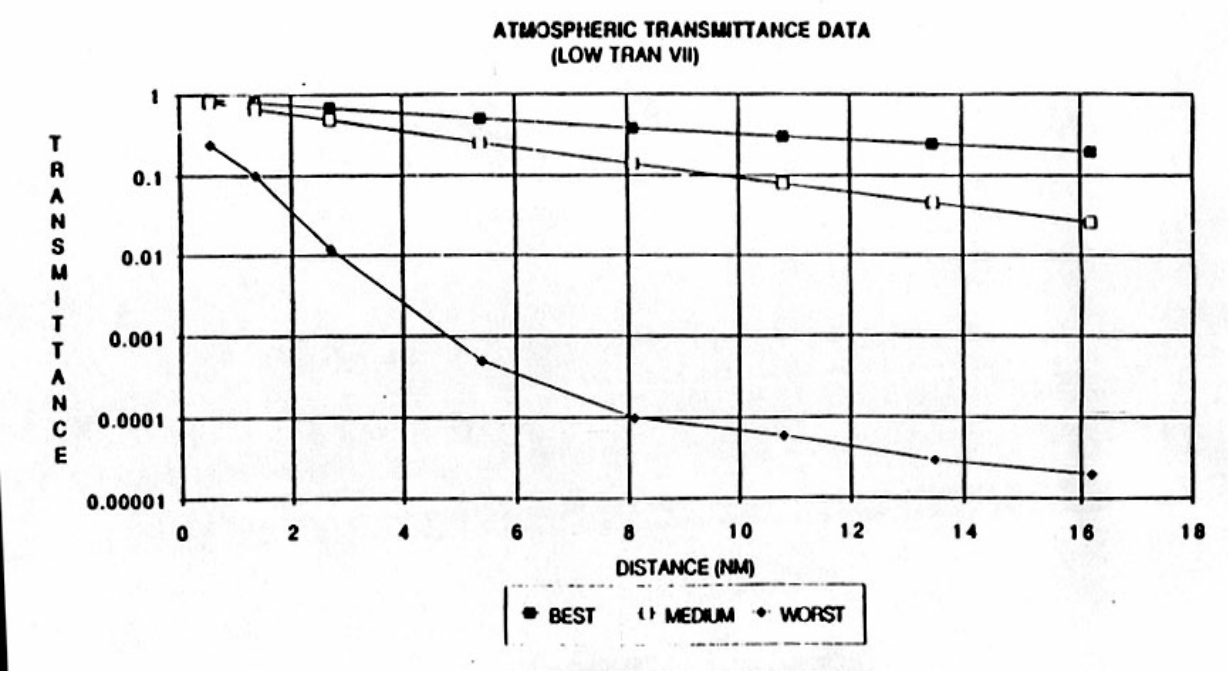

Fig. 29. Average transmittance (8-12 micrometers).

\section{Scene Characteristics}

The characteristics of scene play an important role in detection. ${ }^{5}$ If the intrinsic contrast between target and background is low, the target will be difficult to detect. The presence of other objects in the field of view (clutter) makes it necessary for an operator to distinguish between these objects and the target. Target size is also important in imaging and detection. If the target subtends an angle smaller than 1 degree, then the threshold contrast of the human observer begins to degrade. Similarly for imaging systems and detectors, if the target size is smaller than one pixel, it will be more difficult, or even impossible, to detect.

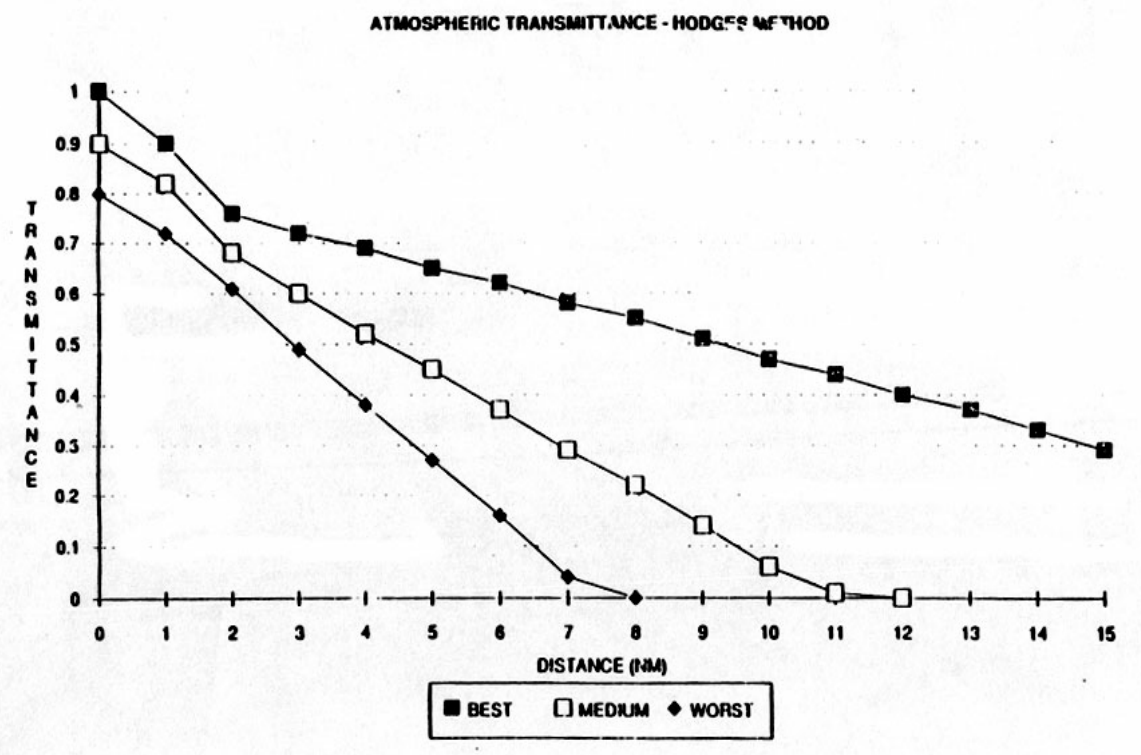


Fig. 30. Approximate transmittance using absolute humidity.

System Characteristics

Characteristics of the imaging system itself are important. A wide field of view is more likely to contain clutter, and the target may not be recognizable if it only influences one or two pixels in the display. A narrow field of view has the obvious disadvantage of covering a smaller area. DC and AC coupled systems are affected somewhat differently by variation in radiance. A DC coupled system is one in which display brightness levels correspond directly to apparent scene radiances. Such a system acts much like human vision. In an $\mathrm{AC}$ coupled system, display brightness depends on the range of the received radiances and not on their absolute values. Path radiance, therefore, is not a factor. If the path radiance is very high, the target image may be buried in the noise. Most thermal imaging systems are AC coupled. A hot object in the scene or a high-temperature smoke cloud in the field of view can increase the range of radiances in the scene so much that the target and its background have almost the same brightness level on the display. In such a situation, the target may be difficult to detect, even though contrast, transmittance, and contrast transmittance are all high. Imaging and detection systems inherently have a signal-to-noise ratio that limits system sensitivity.

\section{Human Variability}

The human operator must be considered as an element in the system. No two people will respond exactly the same way under all circumstances. Indeed, the same person may respond differently at different times. The presence of a human operator, therefore, introduces an element of statistical variation into the problem of target detection and identification.

\section{Image Intensifiers}

The primary factors affecting the performance of image intensifiers include atmospheric transmission, night sky condition, target contrast, clutter, and image intensifier generation. Night vision devices using image intensifiers also have a number of limitations which include reduced visual acuity (vision), limited field of view, visual noise, and degraded depth perception. 


\section{Atmospheric Transmission}

As has been previously discussed, absorption by atmospheric constituents, air temperature, relative humidity, barometric pressure, and obscurants such as fog, haze, and smoke have spectral dependant effects upon the atmospheric transmission. GEN II and GEN III devices operate in different spectral regions; GEN II from $480 \mu \mathrm{m}$ to $850 \mu \mathrm{m}$ and GEN III from $625 \mu \mathrm{m}$ to $900 \mu \mathrm{m}$. GEN II and GEN III devices also differ in their sensitivity over these spectral regions; GEN II has a relative low sensitivity and GEN III has a relatively high sensitivity (see Figure 9). From Figure 27 it can be inferred that GEN II devices will penetrate fog and haze to a lesser degree than GEN III devices, because fog has a minimum transmittance at about $450 \mu \mathrm{m}$ to $500 \mu \mathrm{m}$. Likewise from Figure 28, haze has a minimum transmittance at about the same spectral point.

\section{Night Sky Condition}

GEN III devices were designed to operate in lower light conditions than GEN II devices; specifically starlight versus moonlight conditions. From Figure 19, it can be seen that moonlight contributes significantly to the light available in the $450 \mu \mathrm{m}$ to $850 \mu \mathrm{m}$ spectral region. For a relatively full moon, there is more light energy in the $450 \mu \mathrm{m}$ to $600 \mu \mathrm{m}$ spectral region than the $650 \mu \mathrm{m}$ to $900 \mu \mathrm{m}$ region. However, for starlight only and for a $1 / 4$ moon (with the exception of a spectral peak at $550 \mu \mathrm{m}$ ) there is more light energy in the $800 \mu \mathrm{m}$ to $900 \mu \mathrm{m}$ spectral region than in the $450 \mu \mathrm{m}$ to $600 \mu \mathrm{m}$ region. Coupled with the GEN III's high sensitivity, this allows the GEN III devices to operate "better" in the lower "natural" starlight and low moonlight conditions. It is important to realize, as explained later, than this may not be true if the ambient light conditions are not dominated by starlight and moonlight.

Both GEN II and GEN III night vision devices incorporate automatic gain control. This automatic gain control limits the illuminance of the devices display. This provides various benefits including prevention of output surges, and minimization of light adaptation. A clamp voltage mechanism is present to protect against excessively bright light sources (e.g. flares, search lights, etc.) Typically, the display will reach full illuminance at a light level corresponding to a 1/4 to 1/2 moon night. At low scene illumination (e.g. no moon), the relatively low signal to the intensifier results in a grainy image with noticeable scintillations (i.e. bright sparkles); this image, however, is about 2000 times brighter than the scene would appear to the unaided eye. As illumination levels increase, the graininess and scintillations become steadily less apparent because of the increasing signal. The image, in turn, correspondingly increases in brightness so that the approximate $2000 \mathrm{X}$ amplification factor is maintained. At approximately $1 / 4$ moon illumination, the average image brightness reaches the maximum level set by the intensifier's power supply. As illumination increases from this point, graininess and scintillations continue to diminish because of increasing signal, but the average brightness remains constant. This trend continues for illumination until twilight conditions.

Throughout all these illuminations, scene contrast is determined exclusively by the specific reflectance of terrain features and by the amount of shadowing; image contrast, in turn, maintains a fixed relationship to the scene contrast. Perception of fine detail is limited primarily by graininess and scintillations at low illumination, and by scene contrast at high illumination. Aided visual acuity typically increase smoothly for $20 / 120$ at the lowest natural scene illumination (e.g., starlight under forest canopy), to 20/40 at full moon conditions.

\section{Contrast}

In several studies, it was found that image contrast, which is related to scene contrast, was a differentiating factor between GEN II and GEN III image intensifiers. Image contrast and scene contrast is defined as the difference in brightness between an object and its surrounding background where an object with 5\% contrast is low contrast and difficult to "see" whereas an object with $90 \%$ contrast is high contrast and easy to "see." (Technically, contrast is a complex quantity which is dependant upon the 
spectral radiance of the background and object.) Some contrast values for GEN II, GEN III and human eyes under clear starlight illumination are given in Table 4.

As can be seen from Table 4, the contrast of an object frequently varies between night vision device of different generations as a result of their inherent spectral sensitivity differences. Figure 31 shows how the visual acuity (or angle of resolution) varies with generation of image intensifier under various night sky conditions. The relationship between visual acuity and the ability to detect and/or recognize a target is a complex relationship which varies from individual to individual and device to device. For the purposes of this report, a linear type relationship between visual acuity and the ability to recognize a target will be assumed.

A study (Kotulak \& Rash, 1992) ${ }^{6}$ has shown that visual acuity degrades more rapidly with decreasing night skylight with second generation devices than it does with third generation as illustrated by Figures 32 and 33. Likewise, Figure 34 shows that visual acuity degrades more quickly with decreasing night skylight when contrast is low than when contrast is high. This effect is more pronounced with second generation devices than with third generation devices as shown in Figure 35.

Table 4. Contrast Values for Various Systems, Targets, and Backgrounds for Clear Starlight Illumination

\begin{tabular}{|l|l|c|c|c|c|c|c|c|}
\hline System & \multicolumn{1}{|c|}{ Target } & $\begin{array}{c}\text { Green } \\
\text { Grass }\end{array}$ & $\begin{array}{c}\text { Dead } \\
\text { Grass }\end{array}$ & $\begin{array}{c}\text { Dry } \\
\text { Road }\end{array}$ & $\begin{array}{c}\text { Green } \\
\text { Foliage }\end{array}$ & $\begin{array}{c}\text { Pine } \\
\text { Needles }\end{array}$ & Sand & A \\
\hline GEN II & $\begin{array}{l}\text { Soldier in } \\
\text { Fatigues }\end{array}$ & 44 & 39 & 57 & 50 & 23 & 47 & 43 \\
\hline GEN III & $\begin{array}{l}\text { Soldier in } \\
\text { Fatigues }\end{array}$ & 49 & 28 & 49 & 56 & 35 & 33 & 42 \\
\hline Eye & $\begin{array}{l}\text { Soldier in } \\
\text { Fatigues }\end{array}$ & 24 & 57 & 70 & 18 & 91 & 69 & 55 \\
\hline GEN II & $\begin{array}{l}\text { O.D } \\
\text { Paint }\end{array}$ & 9 & 1 & 31 & 19 & 24 & 14 & 16 \\
\hline GEN III & $\begin{array}{l}\text { O.D. } \\
\text { Paint }\end{array}$ & 7 & 30 & 8 & 21 & 19 & 21 & 18 \\
\hline Eye & $\begin{array}{l}\text { O.D. } \\
\text { Paint }\end{array}$ & 27 & 59 & 71 & 21 & 84 & 70 & 55 \\
\hline GEN II & $\begin{array}{l}\text { Camouflage } \\
\text { Paint }\end{array}$ & 4 & 5 & 27 & 15 & 31 & 10 & 15 \\
\hline GEN III & $\begin{array}{l}\text { Camouflage } \\
\text { Paint }\end{array}$ & 4 & 34 & 5 & 15 & 23 & 25 & 18 \\
\hline Eye & $\begin{array}{l}\text { Camouflage } \\
\text { Paint }\end{array}$ & 6 & 47 & 63 & 1 & 95 & 62 & 45 \\
\hline
\end{tabular}

In summary, visual acuity, which is related to detection ranges and detection probability, with both generations of night vision devices declines with decreasing night skylight and with diminishing target contrast. In addition, theoretical visual acuity (detection range and probability) is consistently better with third generation devices than it is with second generation devices. However, the difference in visual 
acuity between generations widens with decreasing night skylight and with declining target contrast. Furthermore visual acuity degraded more rapidly with decreasing night sky light as target contrast was lowered.

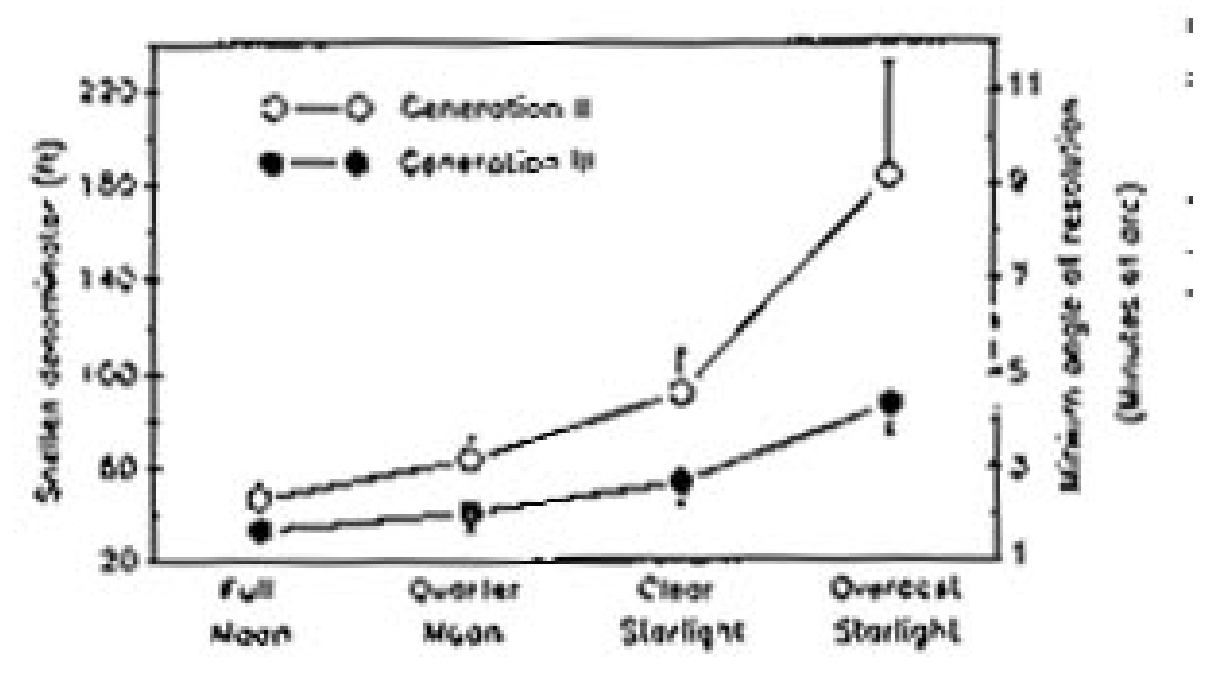

Fig. 31. Visual acuity as a function of night sky condition and generation of night vision goggle with high contrast targets.

An additional study (Miller, Provines, Block \& Tredici, 1984) ${ }^{7}$ has shown that in some instances GEN II devices provide better contrast than GEN III devices. This study tested the AN/PVS-5A (GEN II) against the ANVIS (GEN III). As fully anticipated, the testing of the night vision devices revealed that the GEN III device gave sharper visual resolution and brighter images. However, when a test scene consisting of a semi-paved road meandering through scrub grassland and small trees was viewed, the GEN II device was preferred by personnel because they could "see" the roadway whereas they could not "see" it with the GEN III device. The cause of this phenomenon is not easily surmised, but a plausible explanation can be given. The reasons that the roadway could not be seen by the GEN III device was that the photocathode tube was 


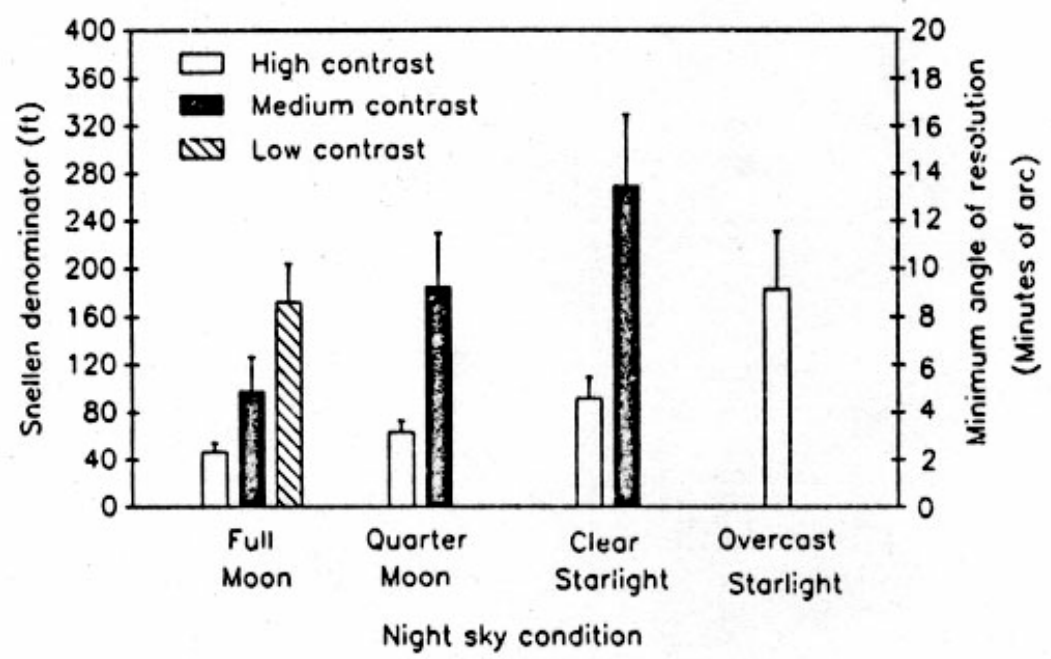

Fig. 32 Visual acuity with generation II devices

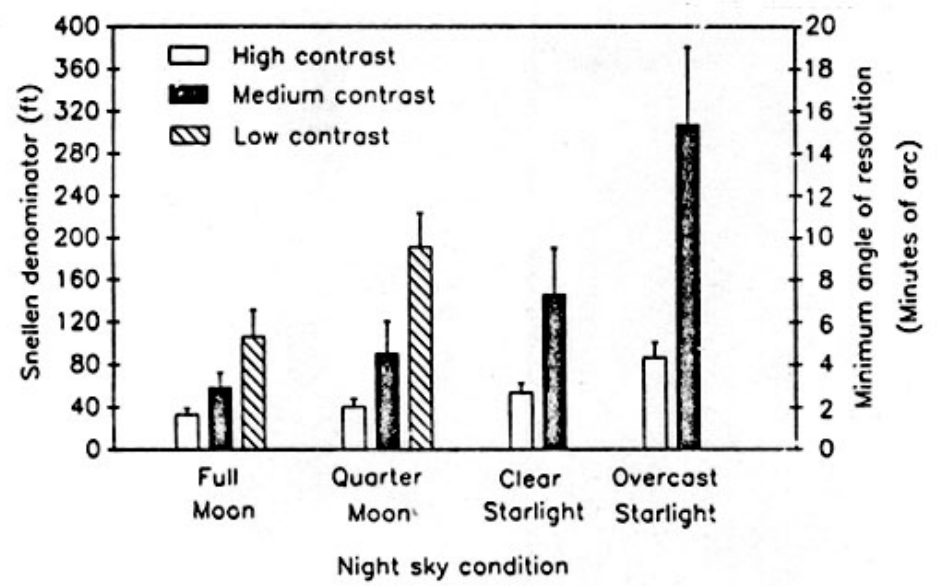

Fig. 33. Visual acuity with generation III devices.

equally sensitive to the road and its immediate surrounding foliage. Thus, there was no contrast gradient between the road and its surroundings to allow visual discrimination. This is undoubtedly due to the enhanced response of the GEN III in the red and IR end of the spectrum. The semi-paved road surface was probably weakly reflecting starlight from the night sky, which contained a great deal of red and IR wavelength. The GEN III tube greatly intensifies this light so that a bright output was obtained which apparently blended in with the output from the surrounding terrain. The terrain and foliage were reflecting both mid and long visible wavelengths that also elicited a bright response from the GEN III tube. Accordingly, there was no detectable difference in contrast between the road and the surrounding area. Hence, the personnel were unable to discriminate the presence of the road with the GEN III device. On the other hand, the GEN II device could discriminate the roadway because its photocathode tube is not as sensitive to the weak red and IR that the road reflected. Hence, the radiation reflected from the road surface did not elicit a response from this tube so it was seen as essentially dark. However, the 
surrounding terrain and foliage were brightly visible to the GEN II tube because its peak sensitivity is in the mid-visible wavelengths. Thus, the road was visible as seen through the GEN II device because of the

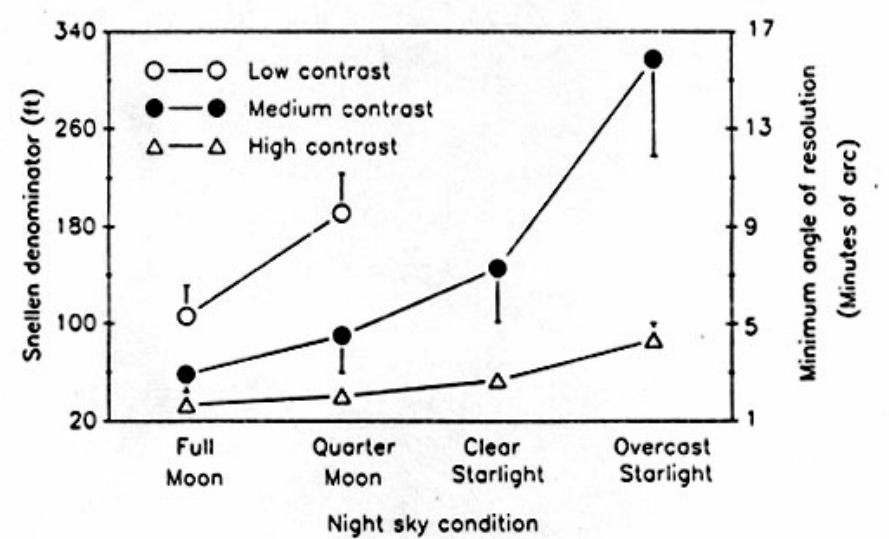

Fig. 34. Visual acuity as a function of night sky condition and target contrast with generation III night vision goggles.

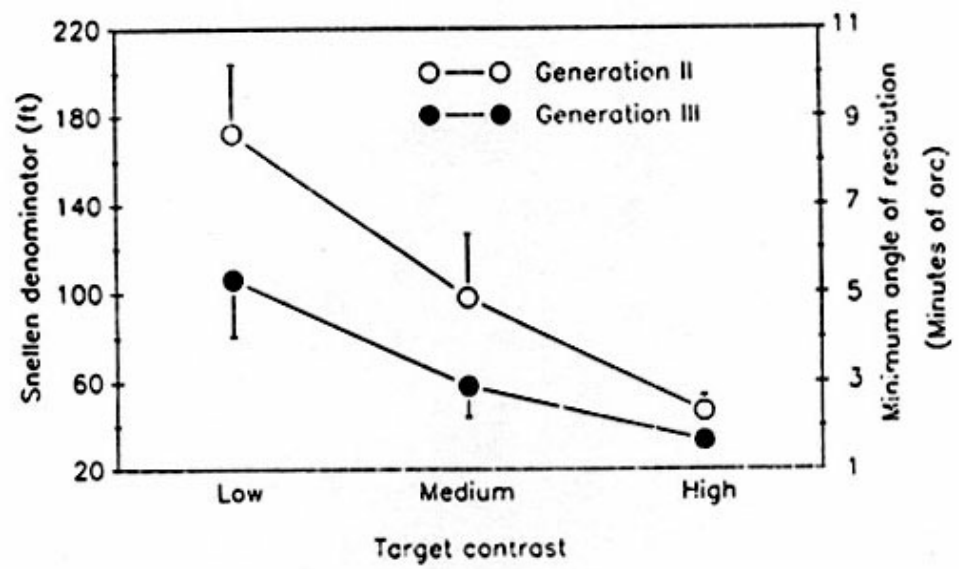

Fig. 35. Visual acuity as a function of contrast and night vision goggle generation under simulated full moon radiance.

contrast gradient that existed between it and the surrounding area. In effect, you could detect the presence of the roadway because its photocathode tube was not sensitive to the road, but was sensitive to the surroundings. Conversely, you could not see the roadway with the GEN III device because its photocathode tube was equally sensitive to the road and surroundings; hence no contrast difference could be discriminated.

A third study (Wiley, 1989) ${ }^{8}$ examined the differences between monocular (AN/PVS-5 with left tube removed), biocular (AN/PVS-7A and AN/PVS-7B), and binocular (AM/PVS-5) night vision devices in regard to target contrast. The results of this study are shown in Figure 36 for full moonlight and Figure 37 for $1 / 4$ moonlight. This study concluded that a $5 \%$ contrast target could not be seen with either GEN II or GEN III devices at the 1/4 moonlight level. In addition, it concluded that a binocular night vision device is best under all circumstances and that a biocular and monocular device are about the same. It also found that vision with the binocular device under low light, low contrast ( $1 / 4$ moon, 35\% contrast) is 
approximately equal to unaided binocular vision (night adapted human eyes) under full moon and 94\% contrast. It also concluded that high levels of contrast are probably unrealistic for real world situations because natural objects seldom present a high contrast; a 35\% contrast is more realistic. Visual acuity (i.e. detectability) for a $35 \%$ contract target is $20 / 70$ under full moon condition and drops to 20/100 under $1 / 4$ moon conditions. Also, the lowest contrast (5\%) targets, which can be represented by a wire against a dark sky, are very difficult to "see." Under full moon conditions, visual acuity is only 20/200 and is much less than 20/400 under 1/4 moonlight.

A fourth study (Bender, Bradford, and Stefanik, 1991) ${ }^{9}$ investigated detection limits with GEN II and GEN III devices in limited time scenarios (15, 7, 4, and 2 second observation windows) in pine canopy, cluttered range, and open meadow conditions. The pine canopy was flat ground surrounded by evergreen trees. The ground had a thin covering of pine needles and the background for the targets generally consisted of tree trunks and the pine needle cover. It featured extremely low scene illumination in conjunction with moderate target contrasts (approximately 30\%). The cluttered range consisted of loosely spaced shrubs/evergreen trees and clump grasses. The background for the targets was generally tree trunks, shrubs, and pine branches. It featured low scene illumination in conjunction with very low (approximately 10\%) target contrasts. The open meadow had been recently cleared by a forest fire. The ground consisted of clump grasses, charred tree stumps, and patches of bare dirt. The background for the targets was mainly

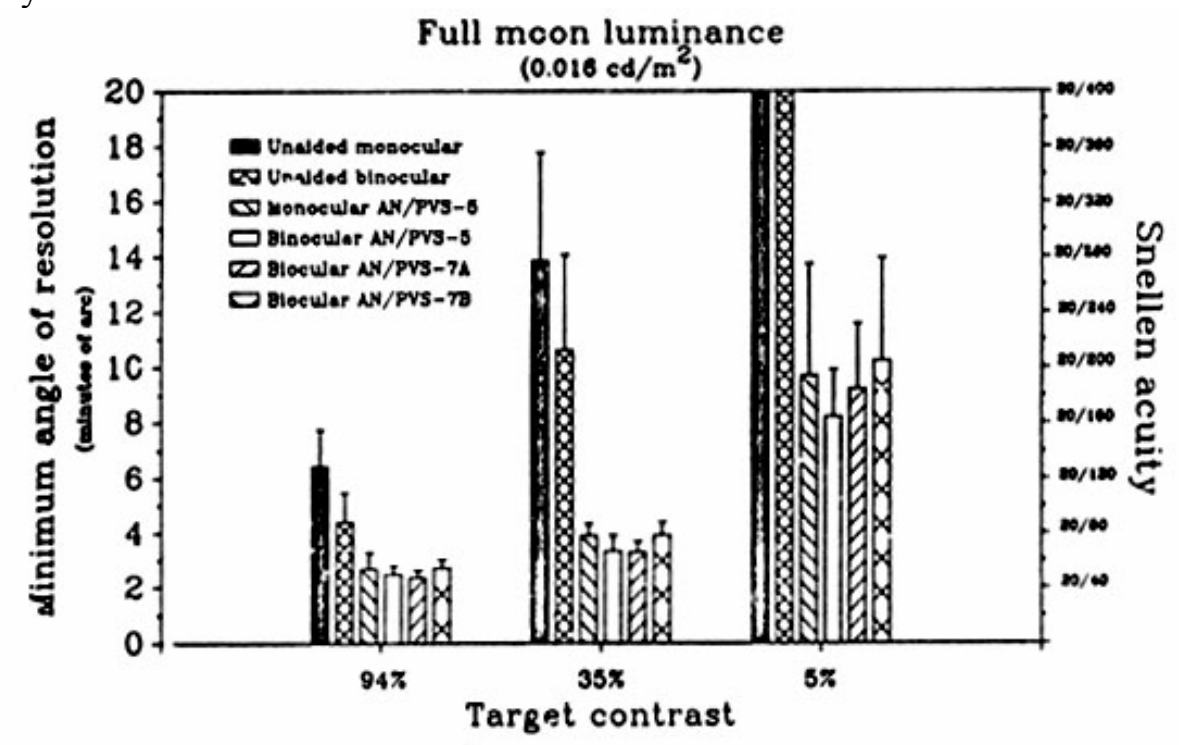

Fig. 36. Visual resolution with simulated full moon ambient luminance and three target contrasts.

the grass/dirt ground covering. It featured low scene illumination in conjunction with relatively high (approximately 60\%) target contrasts.

This study concluded that third generation devices yielded more correct target (human beings) detections and less false detections at each site, the relative differences in detection performance substantially varied from site to site. The third generation devices detected $93 \%$ more targets in the pine canopy, $17 \%$ more targets at the cluttered site, and $6 \%$ more targets at the open site. At the cluttered site, the second generation devices recorded $155 \%$ more false detections than the third generation devices. This was probably more related to low image contrast than to noisier imagery. Histograms which relate the correct detections for each site are presented in Figures 38, 39, and 40 for the canopy site, the cluttered site, and the open site respectively. 


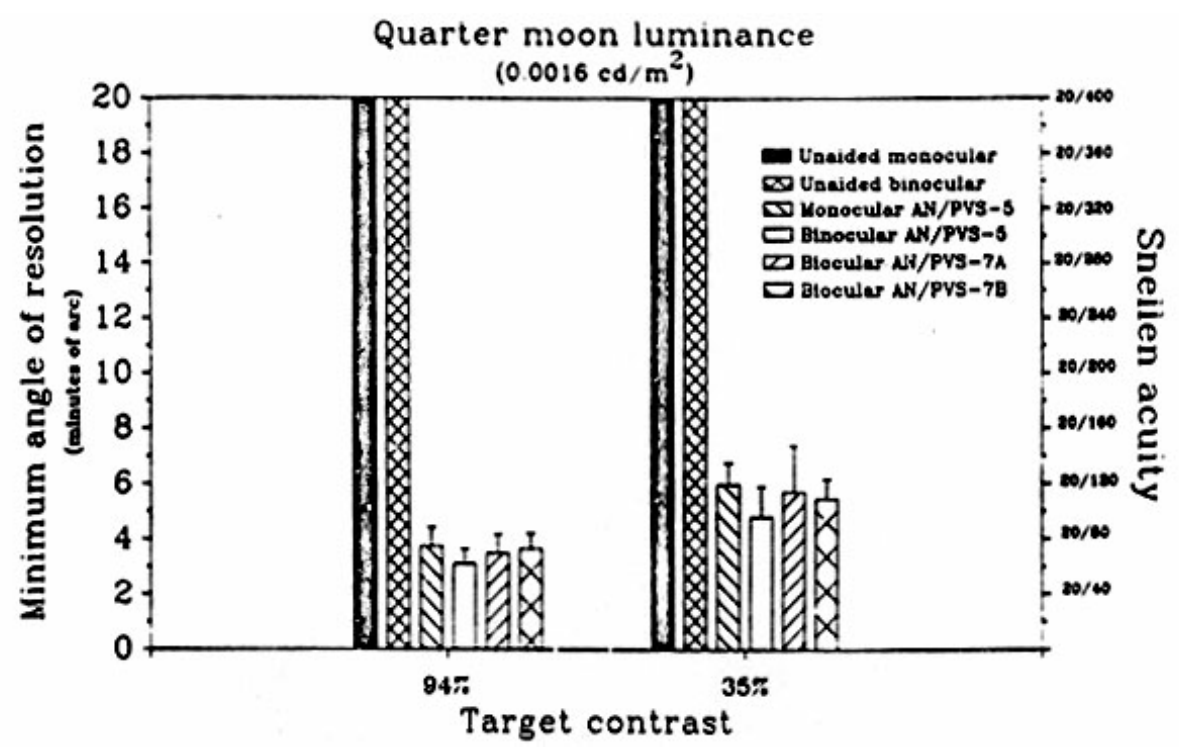

Fig. 37. Visual resolution with simulated quarter moon ambient luminance and two target contrasts.

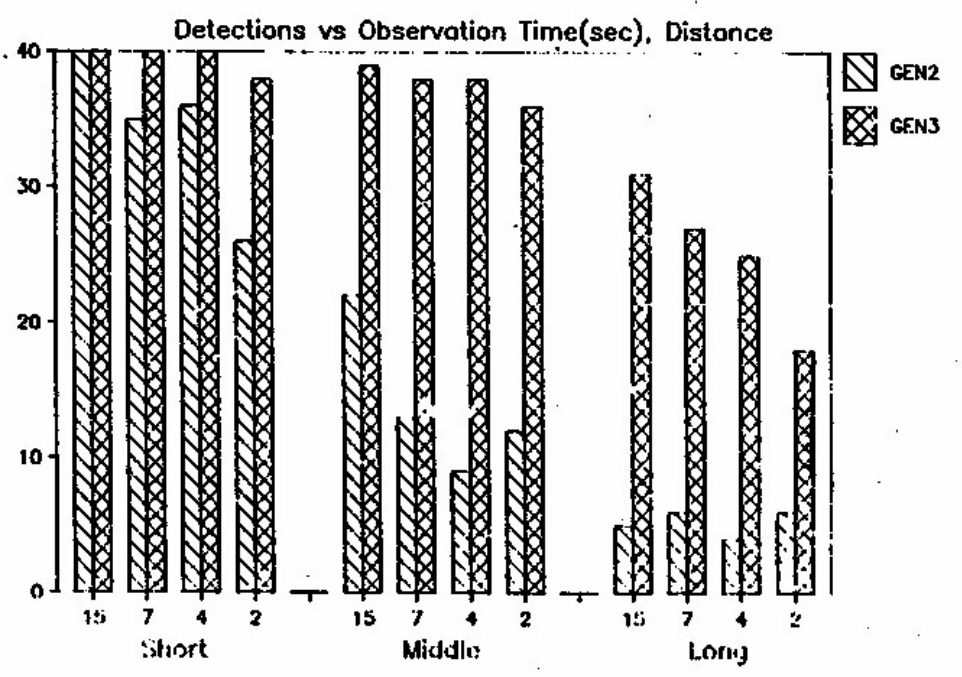

Fig. 38. Histogram of canopy site $(05 / 09 / 88)$.

The prime objective of this study was to ascertain if, as allowed observation time decreased, second generation devices would degrade in detection capability faster than third generation devices. At both the pine canopy and open sites, there were several instances where the second and third generation device detections were essentially identical at the longer observation times. As the observation periods decreased, however, the second generation devices lost $35 \%$ to $45 \%$ of this performance, while the third generation lost $10 \%$ or less. The greatest degradation generally occurred as the observation period decreased from 4

to 2 seconds. Such trends were most dramatic at the pine canopy site, presumably because of its extremely low light levels. In this study, contrast, rather than signal-to-noise, appeared to be an 
overwhelmingly predominant factor in determining second and third generation device performance at the cluttered site.

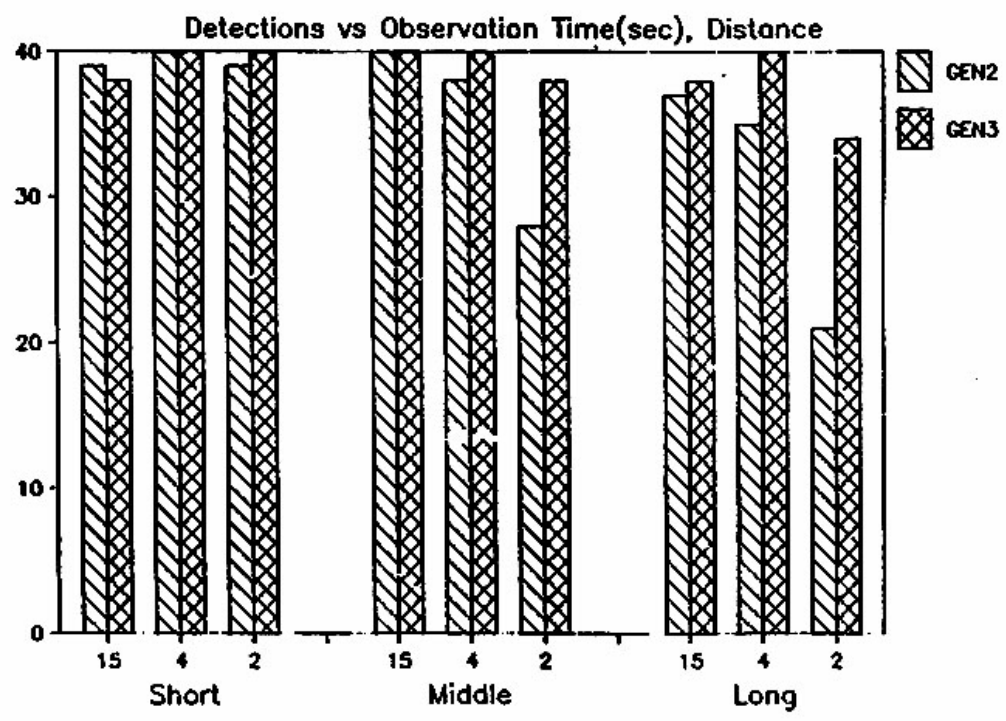

Fig. 39. Histogram of cluttered site $(05 / 10 / 88)$.

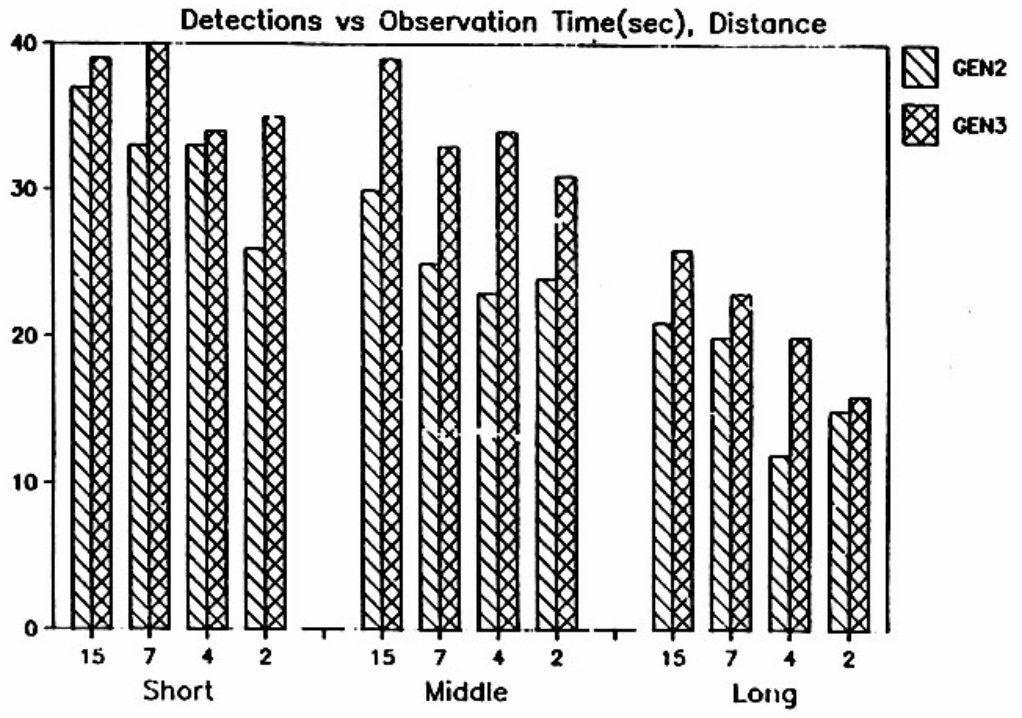

Fig. 40. Histogram of open site $(05 / 08 / 88)$.

The targets were set up at each site to span the $50 \%$ detection probability point. Consequently, cluttered site targets were three times as close as the high contrast open site targets, and were somewhat closer than the moderate open site levels, which put them around six times closer than the canopy site levels. Therefore, image signal-to-noise was only a minor factor in determining detection performance at the cluttered site. 
A study (Whiteley, Task, \& Schueren, 1991) ${ }^{10}$ examined the effects of signal-to-noise ratio (SNR) of GEN III devices on visual acuity (i.e. detection). They determined that increases in intensifier tube SNR resulted in better visual acuity at both quarter moon and starlight illumination for both high and low contrast targets. Improvements in visual acuity with increases in SNR vary depending upon the illumination and contrast as shown in Figures 41, 42, 43, and 44. Doubling the SNR results in a 40\% improvement in visual acuity for low illumination and low contrast. However, the same increase in SNR results in only a $15 \%$ increase in acuity for a $20 \%$ and $90 \%$ contrast target at quarter moon illumination. Therefore, increases in SNR have their greatest impact on visual performance under conditions of low illumination. Although all subjects reported 20/20 visual acuity in daylight, acuity with night vision devices ranged from 20/108 to 20/175 in the most degraded visibility condition (low illumination, low contrast) and from $20 / 42$ to $20 / 65$ in the highest illumination and contrast condition (full moon, $90 \%$ contrast).

Another study (Pollehn, 1988) ${ }^{11}$ concluded that the threshold for target detection is directly proportional to the difference in brightness between the target and the background, divided by the fluctuation in the brightness as measured at the output phosphor screen. This ratio is called the signal-to-noise ratio (SNR). The output brightness is proportional to the current generated at the photocathode, and the SNR is proportional to the square root of this current divided by the noise. Therefore, the threshold for target detection is proportional to the SNR at the output phosphor screen, as given by the square root of the signal current divided by the noise. As an observer moves away from a target, the area of the target's image at the photocathode (and with it the signal current) decreases proportionally to the square of the distance

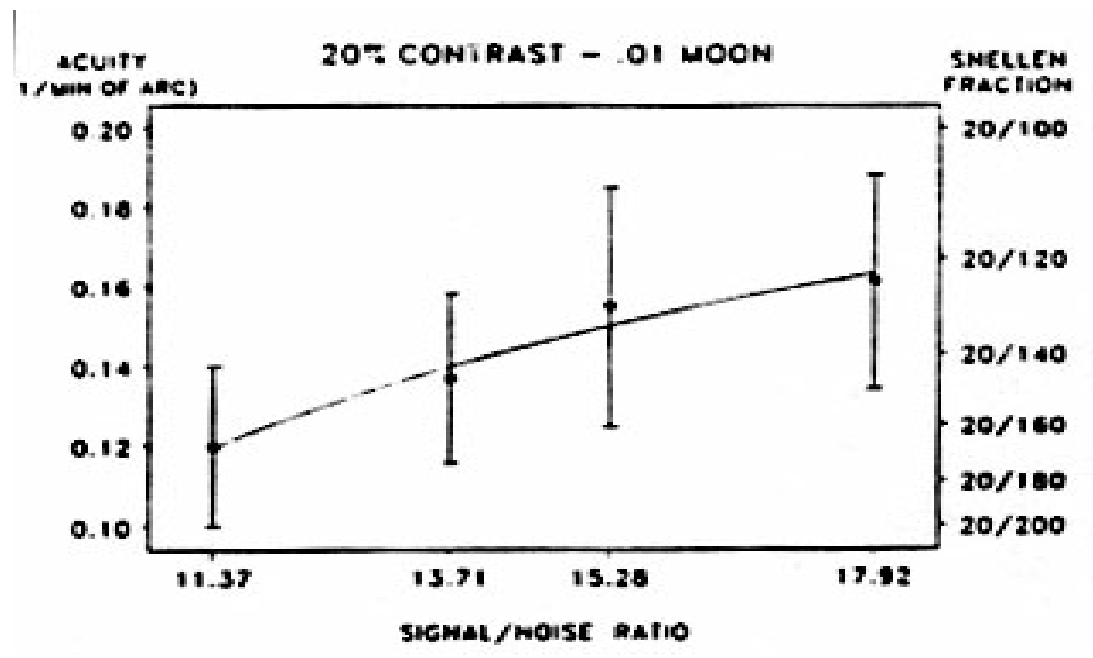

Fig. 41. Visual acuity SNR for low contrast starlight conditions.

between the target and the observer; thus, the SNR decreases with the distance. Since the SNR determines the limit of detection, the detection range is directly proportional to the SNR or to the square root of the signal current. Therefore, the detection range difference between GEN II and GEN III image intensifiers are determined by the differences in their signal currents. 


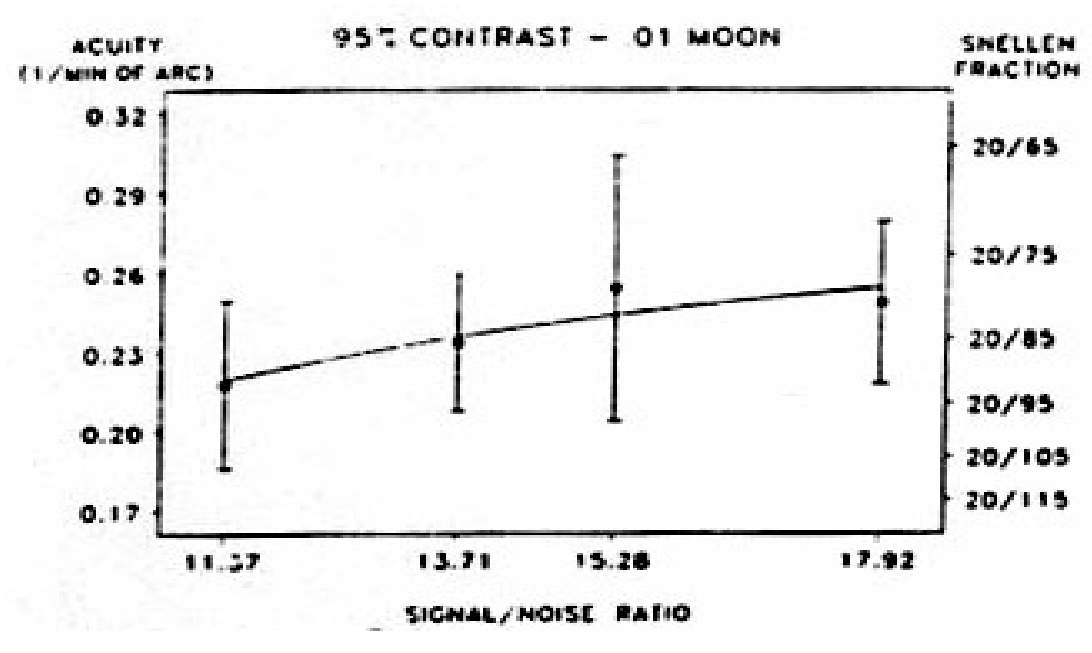

Fig. 42. Visual acuity versus SNR for high contrast starlight conditions.

Another study (Beaton, 1984) ${ }^{12}$ found that the SNR correlates with performance tasks in both hard- and soft-copy displays. SNR is calculated by relating the amount of signal energy to be detected by the observer against a background energy (noise). The relationship is the amount of signal energy divided by the noise energy. Finally, a study (Abel, 1994) ${ }^{13}$ determined that the only metric that correlated significantly with target detection performance was SNR. This suggests that noise levels in the night vision devices actually drive operator target detection performance. This finding is consistent with those of other studies (Riegler, Whitely, Task \& Schueren, 1991), ${ }^{10}$ who found that as the SNR of night vision devices

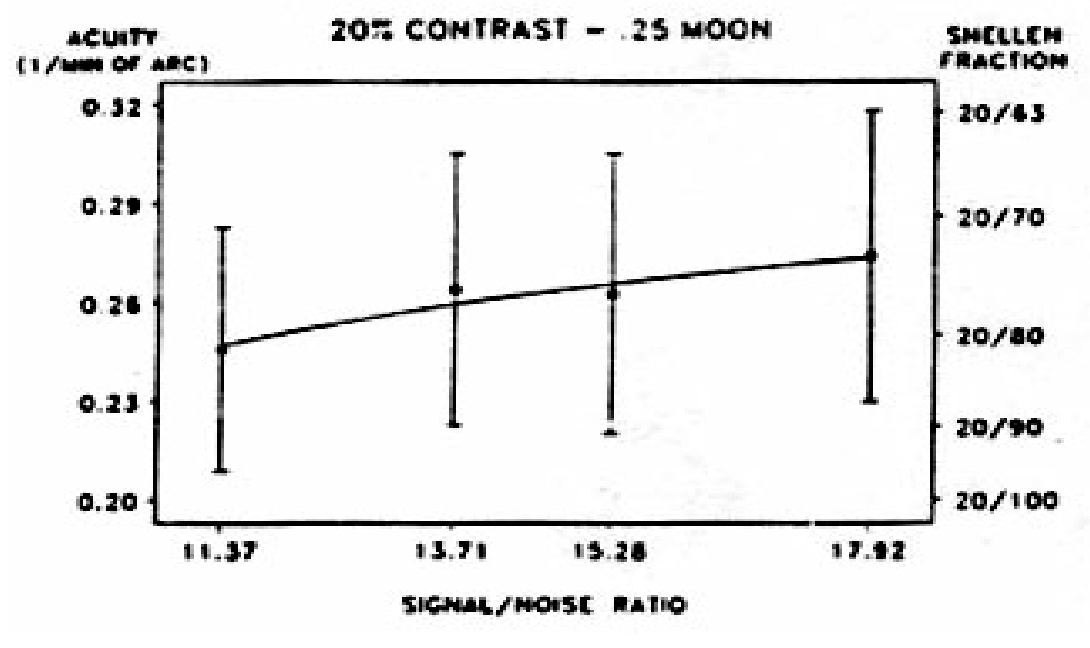

Fig. 43. Visual acuity versus SNR for low contrast quarter moon conditions.

improves so does performance and (Beaton, 1984) ${ }^{12}$ that SNR was the best performance predictor when noise in a system was a consideration. This also makes sense from a psychophysical, signal-to-noise perspective, as the signal strength increases, it is easier to pick it out from a noisy background. However, for target recognition, resolution is most significant. One would expect recognition performance to improve as system resolving power improved. 


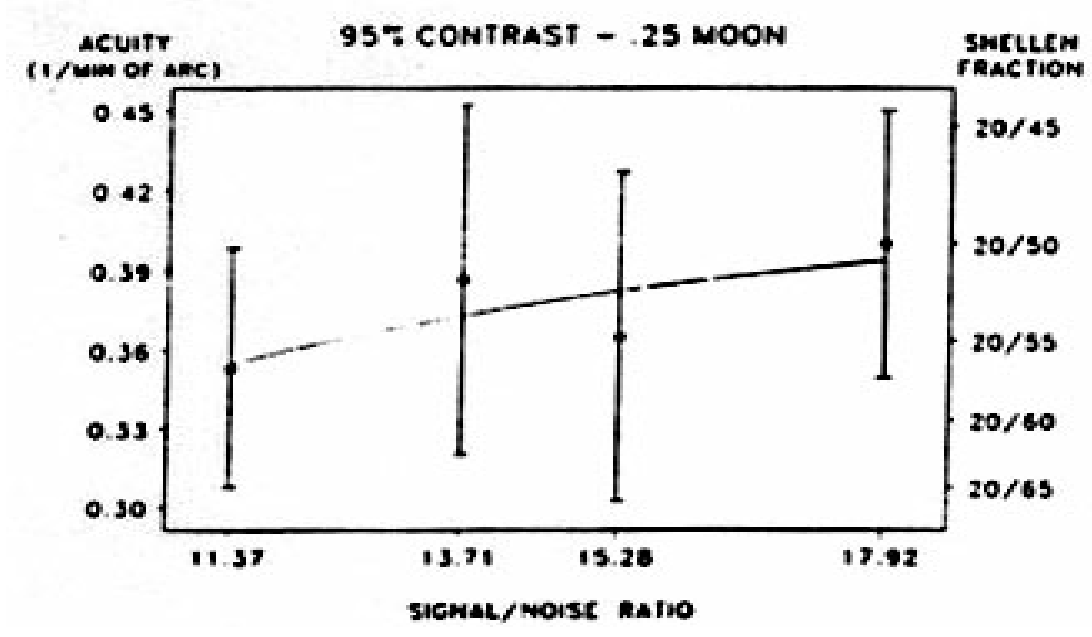

Fig. 44. Visual acuity versus SNR for high contrast quarter moon conditions.

\section{Clutter}

The effects of clutter on target acquisition have been studied quite extensively (Schmieder, 1982) ${ }^{14}$ Although many of the tests and conclusions of that study were focused on the problem as applied to thermal imaging sensors, they stated that the same principles would apply to an observer performing target acquisition tasks with any imaging device from direct view visual systems to near-IR image intensifiers. Their research concluded that target scenes could be categorized in terms of the signal-toclutter (SCR), or ratio of target contrast to clutter contrast. SCR is calculated as the ratio between targetto-background contrast and the contrast among different background elements, and acquisition range is reduced with increasing SCR. Scene complexity is categorized as "none," "low," "moderate," or "high". For instance, a scene has no complexity if it is virtually uniform on a scale comparable to the target size. Noncomplex scenes require very little resolution for target detection. On the other hand, highly complex scenes contain many confusing objects and/or patterns in the target vicinity that may be mistaken for targets. Scene complexity is strictly a function of spatial content, not of contrast. The denominator of $\mathrm{SCR}$, the background clutter contrast, is stratified by complexity so that a given clutter contrast results in a lower SCR with increasing complexity. Although this method has only been implemented for imaging IR systems, it should be applicable to systems operating in the shorter visual and near-IR (i.e. 0,7 - 1.0 microns) wavelength regions where reflected radiance dominated thermal emission. For these systems SCR can be determined from the brightness contrast between target and background and between background clutter features. Similarly, scene complexity can be incorporated to describe spatial clutter content and to modify the clutter contrast function in the denominator of the SCR (Snapp, Gouveia, Hilton, 1992) ${ }^{15}$

Secondary factors affecting the performance of image intensifiers include artificial lighting, target motion, and self motion. One study (Pollehn, 1988) ${ }^{11}$ has examined the performance degradation of all three of these effects.

\section{Target Motion \& Self Motion}

In this study, the primary purpose was to compare GEN II and GEN III image intensifiers with respect to detection ranges of a man target. The image intensifier systems used were the AN/PVS-5, AN/PVS-7 and 
AN/PVS-6. The initial test was for a walking observer to detect a stationary man target. Variability in the test data was too diverse to draw conclusions. During test on the same night and with the same equipment, detection ranges under medium contrast varied between $30 \mathrm{~m}$ and $140 \mathrm{~m}$. The available data could not confirm the reason for the large variability. Insufficiently trained observers and changing illumination conditions are suspected.

A second test was run with better trained observers and the test methodology was changed to detect a moving man target by a stationary observer. The results from this test were more conclusive. However, this experience illustrates the difficulty in detecting a stationary target while moving, especially by untrained or limited trained personnel as might be found in third world countries. It is much easier to detect a moving target from a stationary observation position than a stationary target by a moving observer.

\section{Artificial Lighting}

Of the secondary factor affecting image intensifier performance, artificial lighting can affect the generation to generation performance the most. The same study illustrated the effects of artificial lighting on image intensifier performance. Figure 45 shows the illumination conditions which can be encountered even at relatively large distances $(15 \mathrm{~km})$ from populated areas. The light illuminating the scene is a mixture of more or less attenuated light from the night sky, starlight, atmospheric sky glow, and reflected and scattered street lighting. Figure 46 shows the spectral distribution of a clear night sky and two commonly used streetlights. The power levels or the irradiance for these sources of illumination have been normalized for the same light level. Streetlights are designed to have most of their output power in the wavelength region where the human eye (or the photometer) is most sensitive. The spectral irradiance of clear starlight, however, increase sharply and is greatest beyond $0.7 \mu \mathrm{m}$ (see Figure 18). This is the wavelength region (beyond $0.7 \mu \mathrm{m}$ ) where GEN III image intensifiers are most sensitive, giving this technology the ability to capture the available higher radiation of the night sky (see Figure 9). It is also the region where target reflections tend to be very high.

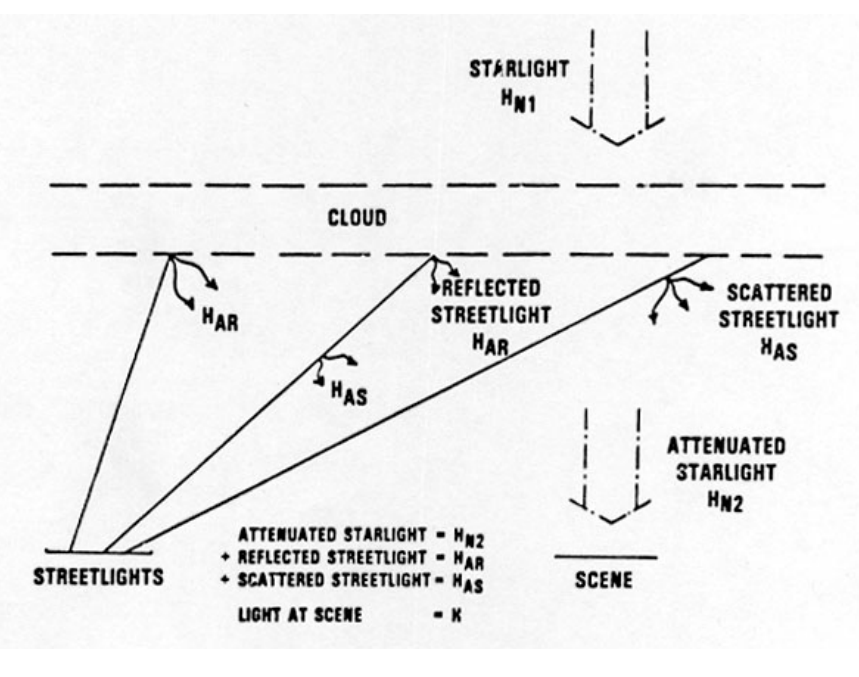

Fig. 45. Illumination conditions.

In order to determine the expected performance difference between GEN II and GEN III image intensifiers under various illumination and irradiance conditions (including both natural and artificial 
sources), the signal current generated by each of these devices under these conditions must be determined. The signal

current may be significantly different for GEN II and GEN III intensifiers even though the illumination levels as seen by or measured for the human eye of all the sources are identical. The analysis of this study shows that it takes 60 times more "light" to generate the same signal current in a GEN III image intensifier when the scene is illuminated with a mercury lamp as compared with the pure night sky. For a GEN II image intensifier, the factor is only 9.3 under the same conditions. In other words, keeping the "light" the same, but changing from night sky to mercury illumination, the GEN III signal current will decrease by a factor of 60 whereas the GEN II signal current will decrease by only a factor of 9.3. Figure 47 shows the range increase (ratio) of the GEN III devices over the GEN II devices for these type conditions.

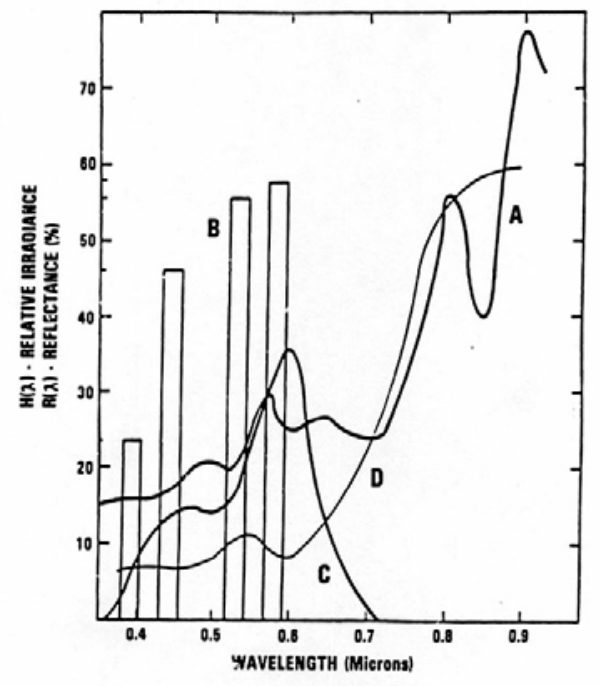

Fig. 46. Spectral irradiance normalized to 1 footcandle illuminance for night sky (A), mercury lamp (B), fluorescent lamp (C), and foliage reflection (D). Some cloud cover was present during all of the testing. During the testing, the cloud cover changed considerably within a short period of time from basically clear sky to close to $100 \%$ cloud cover at high altitude $(6 \mathrm{~km})$. The measured light level nearly doubled when the clouds rolled in. Because clouds do not amplify light, the explanation is that the increase in the measured light level was caused by artificial light from distant sources being reflected from the cloud layer. As a general rule, a $75 \%$ to $100 \%$ cloud cover will attenuate the night skylight by a factor of 10 , and a $38 \%$ to $63 \%$ cloud cover will attenuate by a factor of 3. Using estimates, the percentage of light from the true night sky can be as low as $2.5 \%$ for overcast conditions and high clouds, and as high as $50 \%$ for a clear sky.

In summary, the amount of distantly reflected or scattered artificial lighting present can equalize the performance of GEN II and GEN III devices. In general, GEN III devices perform better than GEN II devices. However, when artificial lighting sources are present, this artificial lighting provides energy in the spectrum band where GEN II devices are more sensitive and therefore, they provide a much better image than they normally would under starlight and moonlight illuminated conditions. 


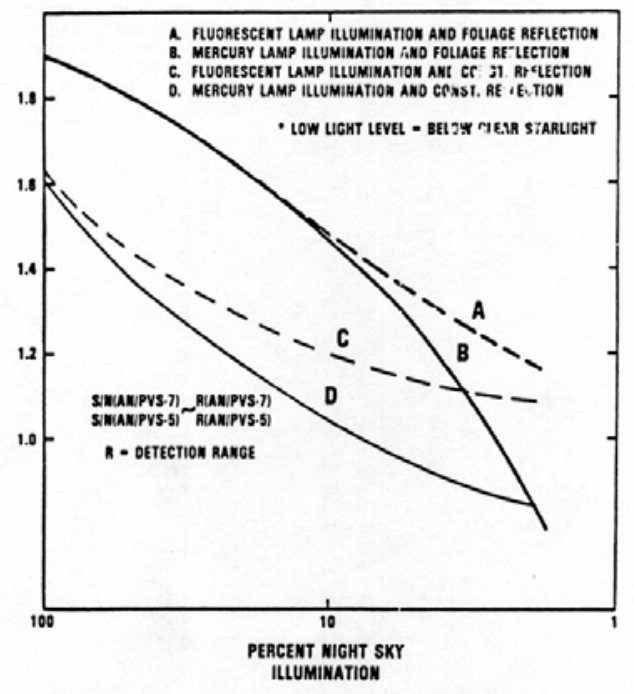

Fig. 47. Low light level range increases of GEN III AN/PVS-7 over AN/PVS-5 as function of percentage of night sky.

\section{Image Intensifier Limitations}

Image intensifier night vision devices have a number of limitations that must be recognized. The first limitation is that they permit at best a visual acuity of 20/40. This acuity can only be reached if the goggles are focused correctly. A study (Biberman \& Alluisi, 1992) ${ }^{16}$ showed that the 20/40 acuity was rarely achieved by operators. The second and third limitations are physical in nature. The second limitation is the field of view of the goggles, which is 40 degrees. The field of view severely limits peripheral vision. This limitation has been likened to looking through two toilet paper rolls, The third limitation is visual noise. Noise appears in two forms: image noise and goggle-induced noise. Goggle induced noise consists of three effects: "sparkle," "blooming," and "halo". Sparkle is caused by random photoelectrons striking the photocathode, it also is referred to as "snow" since it resembles snow falling in front of your car while the headlights are on. Blooming and halo are related noise effects; both occur when bright lights are viewed through the goggles. Blooming, also referred to as "washing out," is caused by the spread of intensified light around the edge of the image on the screen, resulting in a blurred image. Halo is a brighter ring around an image that could cause a less bright image to go unnoticed. The fourth limitation is degraded depth perception while wearing night vision devices. A study (Armentrout, $1993)^{17}$ found that depth perception performance decreases after ambient light is raised above $50 \%$ moon illumination. Two studies (Armentrout, 1993 \& Riegler, Whitely, Task, \& Schueren, 1991) ${ }^{17}$, 10 suggest that as illumination levels increase above $50 \%$ moon illumination, there is a decrease in the resolving power and signal-to-noise ratio (SNR) of the devices that degrades performance.

\section{Resolution}

Resolution is a system parameter that is related directly to the edge sharpness on the phosphor image screen. It is also related to display quality in that the smaller the discernible details on the display, the higher the quality of the display. Because resolution is a system parameter, one would expect it to be constant for a given device. A study (Armentrout, 1993) ${ }^{17}$ found that for ANVIS-6 devices, resolution was not constant across different moon illumination levels. Figure 48 presents the measured resolution of the ANVIS-6 device versus illumination. In the case of ANVIS-6, the best available resolution is 40 line pairs per millimeter $(\mathrm{LP} / \mathrm{mm})$. If one does not take static noise such as halo and blooming into account, 
then resolution theoretically remains at $40 \mathrm{LP} / \mathrm{mm}$. This is not how the night vision devices work. As illumination decreases, the gain in the goggles automatically increases, resulting in more goggle-induced noise. The increase in noise results in a decrease in resolution. Resolution is measured by looking at an Air Force standard three-bar chart to determine the smallest spatial frequency (line spacing) that can be viewed as separate lines through an imaging system. Theoretical resolutions for different illumination are shown in Figure 49. The limiting resolution of a system is the spatial frequency at which an observer can no longer discriminate the light and dark bars of a square-wave image (Snyder, 1985) ${ }^{18}$ Resolution serves as the best performance predictor in target recognition.

\section{Infrared Devices}

The primary factors affecting the performance of infrared imagers include the detector materials in the imager's sensor, the wavelength that the detector material is sensitive to, the noise equivalent temperature (NET), the minimum resolvable temperature (MRT), the minimum detectable temperature (MDT), and resolution.

\section{Detector Materials}

As previously stated, infrared detectors are classified as either photon detectors (generally cooled infrared devices operating in the 3 to $5 \mu \mathrm{m}$ mid IR band) or thermal detectors (generally uncooled infrared devices operating in the 8 to $12 \mu \mathrm{m}$ far IR band). While some detector materials are responsive over wide ranges of both IR radiation and temperature, typically materials are utilized for either one IR band or the other and one temperature range. Table 5 illustrates some of the commonly used infrared detector materials. PtSi led the way to large focal plane array (FPA) sensors. PtSi FPA's of 512 X 512 and 1024 X 1024 are commercially available, although at high $(\$ 100,000)$ cost. PtSi has successfully transitioned into production imagers, with five or more commercial camera vendors adopting this material as the basis for their cameras. In smaller formats of 256 X 256 or 240 X 320 as used in these cameras, PtSi based camera prices, including a mechanical cooler, are in the range of $\$ 30,000$ to $\$ 40,000$. PtSi imagers have very good performance. A typical spectral response at a commercial PtSi detector is shown in Figure 50. InSb is a mature technology which results in highly reliable, cost-effective FPAs for Mid Wave Infrared

(MWIR) applications. The excellent uniformity, responsivity, and spectral response gives the InSb FPA a proven advantage over other materials for high-performance IR systems. 


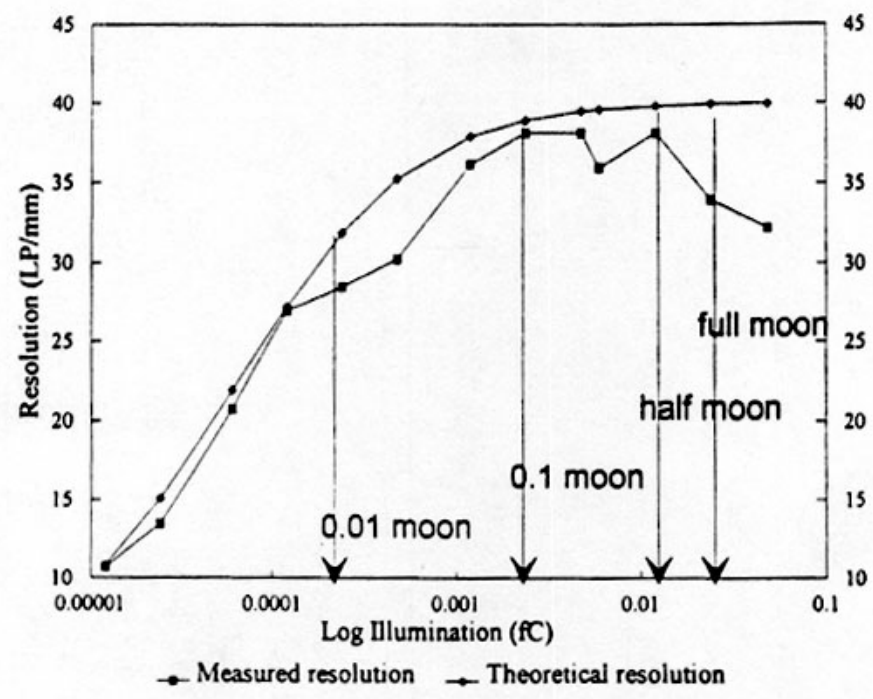

Fig. 48. Measured resolution of ANVIS-6 versus illumination.

$\mathrm{HgCdTe}$ is a material which is sensitive over most of the entire IR bandwidth. $\mathrm{HgCdTe}$ based sensors have been developed which are sensitive in the Short Wave Infrared (SWIR) 1 to $3 \mu \mathrm{m}$, the Mid Wave Infrared (MWIR) 3 to $5 \mu \mathrm{m}$, and the Long Wave Infrared (LWIR) 8 to $12 \mu \mathrm{m}$ ranges. $\mathrm{The} \mathrm{Hg}_{\mathrm{x}} \mathrm{Cd}_{\mathrm{y}} \mathrm{Te}_{\mathrm{z}}$ material is also used in various relative percentage configurations with the relative percent component being identified by the "x," "y," and "z" subscripts. The HgCdTe material can also be used in the cooled

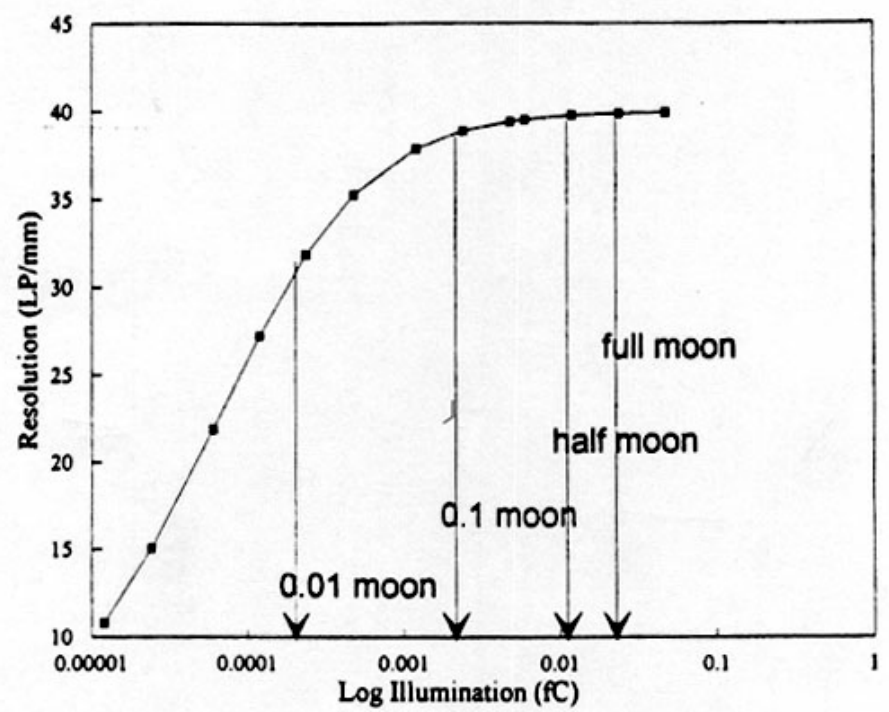

Fig. 49. Theoretical night vision device. 
or uncooled mode. HgCdTe is not as stable as most of the other infrared imager materials. The spectral response of one $\mathrm{HgCdTe}$ detector is shown in Figure 51.

$\mathrm{PbSe}$ and $\mathrm{PbS}$ are high performance detector arrays which can be operated at either room temperature or at $220^{\circ} \mathrm{K}$ using a low power thermoelectric cooler. PZT, BST, and PST are somewhat related materials used in uncooled detectors operating in the 8 to $12 \mu \mathrm{m}$ IR band. PZT has a high transition temperature relative to ambient, and is usually operated in the pyroelectric mode to provide a level of performance which is effectively independent of operating temperature. BST has a transition temperature close to ambient, and when operated in the dielectric bolometer (see Ferroelectric Materials section) mode produces a performance improvement over PZT of a factor of 2 , at the expense of the required temperature stabilization. PST offers even higher performance than BST, at a level approximately 3 times that of PZT.

Table 5. Infrared Imager Detector Materials

\begin{tabular}{|c|c|c|c|c|}
\hline Detector Material & $\begin{array}{c}\text { Operating } \\
\text { Temperature }\end{array}$ & IR Bandwidth & $\begin{array}{l}\text { Focal Plane } \\
\text { Array Size }\end{array}$ & $\begin{array}{l}\text { Commercial } \\
\text { Manufacturer }\end{array}$ \\
\hline $\begin{array}{c}\text { Platinum Silicide } \\
\text { PtSi }\end{array}$ & $\begin{array}{c}77^{\circ} \mathrm{K} \\
\text { (Cooled) }\end{array}$ & $3-5 \mu \mathrm{m}$ & $320 \times 240$ & $\begin{array}{l}\text { Rockwell } \\
\text { Boeing }\end{array}$ \\
\hline $\begin{array}{l}\text { Indium Antimonide } \\
\text { InSb }\end{array}$ & $\begin{array}{c}77^{\circ} \mathrm{K} \\
\text { (Cooled) }\end{array}$ & $3-5 \mu \mathrm{m}$ & $256 \times 256$ & $\begin{array}{l}\text { Hughes } \\
\text { Litton }\end{array}$ \\
\hline $\begin{array}{c}\text { Mercury Cadmium } \\
\text { Telluride } \\
\mathrm{Hg}_{\mathrm{x}} \mathrm{Cd}_{\mathrm{y}} \mathrm{Te}_{\mathrm{z}} \\
\text { (MCT or } \mathrm{CMT})\end{array}$ & $\begin{array}{c}77^{\circ} \mathrm{K} \\
\text { (Cooled) }\end{array}$ & $\begin{array}{l}3-5 \mu \mathrm{m} \\
\text { (Sensitive } \\
2-16 \mu \mathrm{m})\end{array}$ & $\begin{array}{c}640 \text { X } 480 \\
(1024 \text { X } 1024 \\
\text { Custom) }\end{array}$ & $\begin{array}{c}\text { Santa Barbara } \\
\text { Research Corporation } \\
\text { Boeing }\end{array}$ \\
\hline $\begin{array}{l}\text { Lead Sulfide } \\
\quad \text { PbS }\end{array}$ & $\begin{array}{c}220^{\circ} \mathrm{K} \\
\text { (Cooled) }\end{array}$ & $1-3 \mu \mathrm{m}$ & $256 \times 256$ & Litton \\
\hline $\begin{array}{l}\text { Lead Selenide } \\
\text { PbSe }\end{array}$ & $\begin{array}{c}220^{\circ} \mathrm{K} \\
\text { (Cooled) }\end{array}$ & $1-5 \mu \mathrm{m}$ & $256 \times 256$ & Litton \\
\hline $\begin{array}{l}\text { Lead Zirconium } \\
\text { Titanate } \\
\text { PZT }\end{array}$ & $\begin{array}{c}295^{\circ} \mathrm{K} \\
\text { (Uncooled) }\end{array}$ & $8-14 \mu \mathrm{m}$ & $327 \times 245$ & $\begin{array}{l}\text { Lockheed Martin } \\
\text { Boeing }\end{array}$ \\
\hline $\begin{array}{c}\text { Barium Strontium } \\
\text { Titanate } \\
\text { BST }\end{array}$ & $\begin{array}{c}295^{\circ} \mathrm{K} \\
\text { (Uncooled) }\end{array}$ & $8-14 \mu \mathrm{m}$ & $245 \times 328$ & Texas Instruments \\
\hline $\begin{array}{c}\text { Lead Scandium } \\
\text { Titanate } \\
\text { PST }\end{array}$ & $\begin{array}{c}295^{\circ} \mathrm{K} \\
\text { (Uncooled) }\end{array}$ & $8-14 \mu \mathrm{m}$ & $256 \times 128$ & GEC-Marconi \\
\hline
\end{tabular}

\section{Measures of Performance}

In evaluating the performance of infrared imagers, regardless of whether they are cooled or uncooled, there are several parameters which need to be understood and examined. These parameters include Noise 
Equivalent Temperature (NET), Minimum Resolvable Temperature (MRT), Minimum Detectable Temperature (MDT).

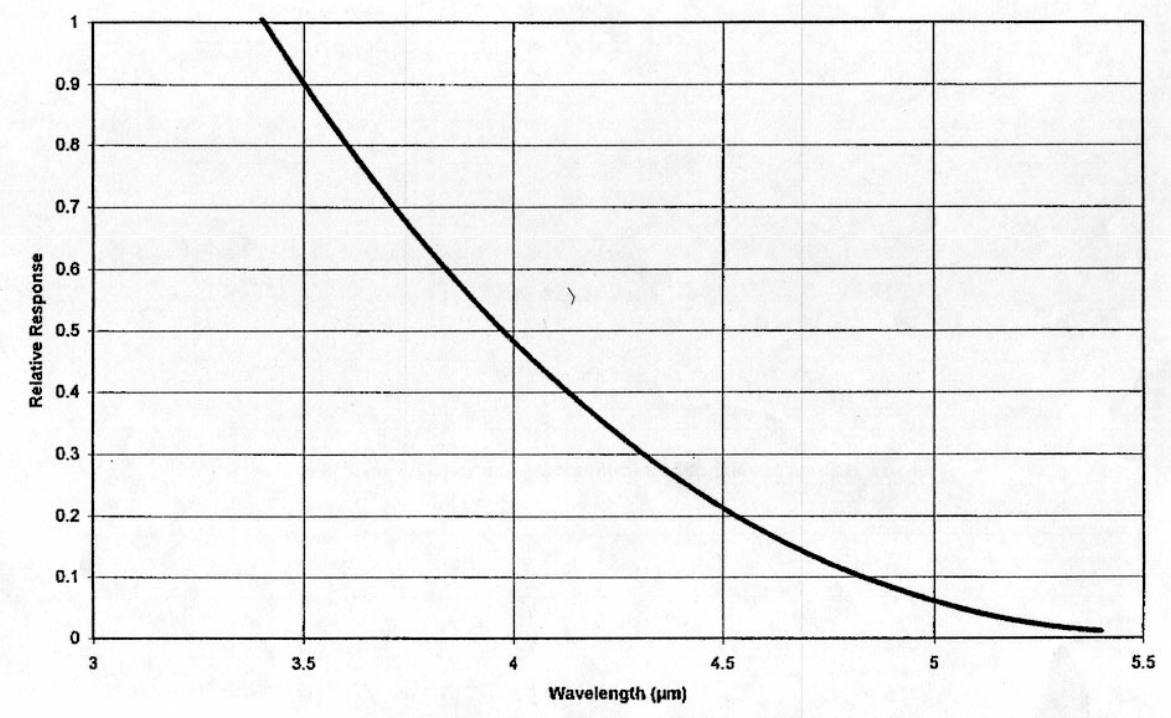

Fig. 50. Spectral response of PtSi detector.

\section{Resolution}

As far as image quality is concerned, J.M. Lloyd, in his classic reference "Thermal Imaging Systems," points out that "In FLIRs, spatial resolution and thermal sensitivity dominate performance." Thermal characteristics of the sensor or the target are insignificant when resolution is the criterion for detection or recognition. In the presence of good weather conditions, and good target-to-background thermal contrast, results will be limited to the best possible determined by the geometry of the situation - eye's resolving power, target's critical dimension, and the viewing distance. Results will degrade as the weather conditions and target contrast degrade. A thermal imaging system has detectors between the optics viewing the target and the target image seen by the eye. These detectors dissect the target into discrete elements according to the angular subtense created by the physical size of the detectors and the effective focal length of the optical elements used to image the target. The angular subtense of a single detector is referred to as the instantaneous field-of-view (IFOV) of the sensor. Therefore, the resolution of a thermal imager is determined by the focal plane array (FPA) and the associated lens optics. The IFOV becomes 


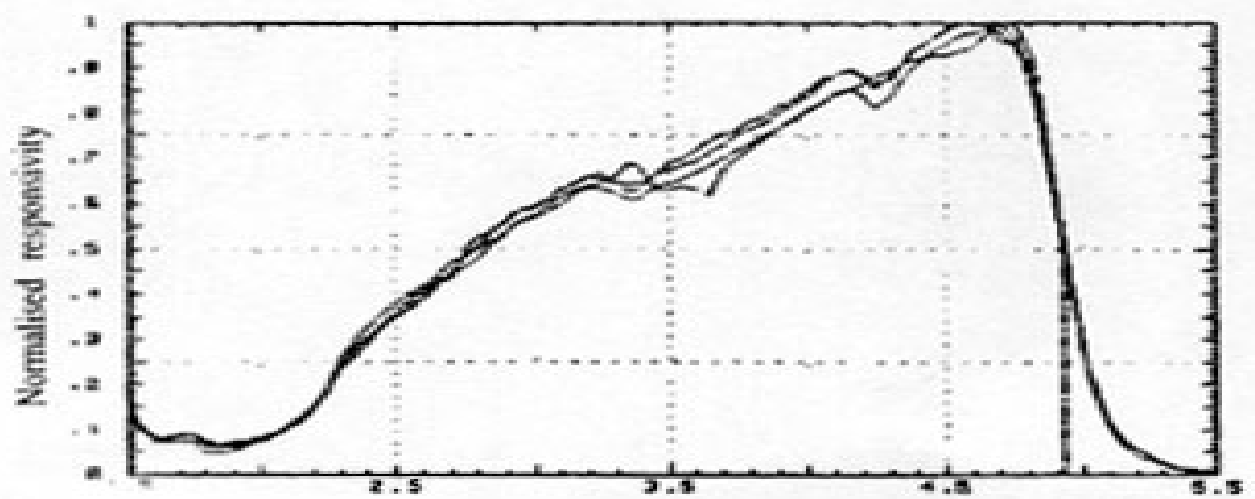

Fig. 51. Spectral response of $\mathrm{HgCdTe}$ detector. 
the limiting link in the geometry if it is larger than the eye's limiting angular resolution. A relationship between angular subtense and target detection limits is shown in Figure 52.

For our purposes of discussion, resolution is the ability to detect two distinct lines separated by a distance equal to the thickness of each line. For $1 \mathrm{X}$ magnification, the human eye can resolve such lines when their image subtenses a solid ( 3 dimensional) angle of $0.377 \mathrm{mrad}$. For 2 line (also referred to as 1 cycle) detection, the image angular subtense would need to be no smaller than the angular period resolvable by the eye, or a spatial frequency of $1 / 0.377$ or $2.65 \mathrm{cycle} / \mathrm{mrad}$. In the ophthalmologists office, and with good object contrast, a person with $20 / 20$ vision can resolve a spatial frequency of 1.7 cycle $/ \mathrm{mrad}$.

\subsection{FT. TARGET}

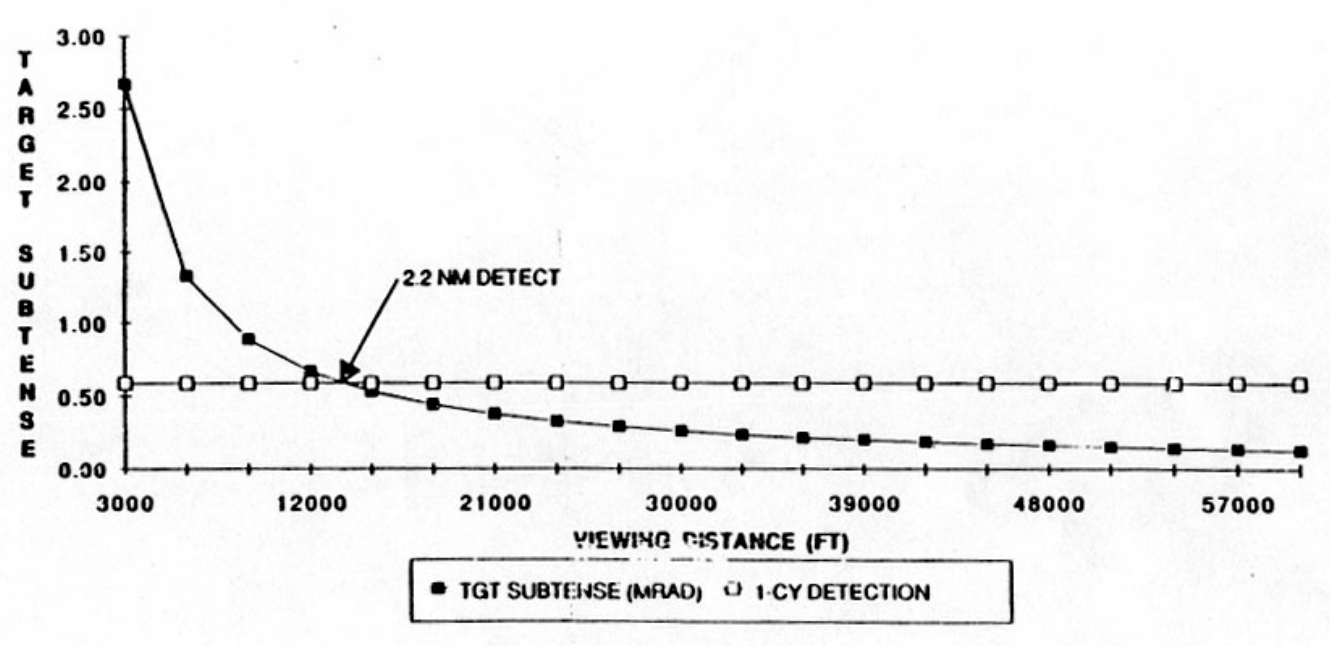

Fig. 52 Detection range limit-20/20 visual acuity.

Therefore, the image angular subtense is $1 / 1.7$ or $0.588 \mathrm{mrad}$. In other words, like a human, the image on the infrared imager must be a certain size in order for the imager to resolve differences in temperature. The required size of the object is determined by the number of FPA elements and the lens magnification in front of the FPA (i.e. the apparent size of the object to the FPA). Therefore, resolution is influenced by distance between the imager and the object. It should be noted that when the optical resolution limit is reached, thermal contrast is not significant. There contrast sensitivity is only of interest at ranges less than the optical limit. It should also be noted that with an infrared sensor, "hot spot" detection (unresolved image) can be achieved at ranges greater than the sensor's optical limit.

\section{Noise Equivalent Temperature (NET)}

Noise equivalent temperature (NET) is the temperature difference between two blackbody sources which produces a signal to noise ratio of one. NET is thus a measure of the signal to noise ratio of the system. Other measurement parameters such as minimum resolvable temperature (MRT) and minimum detectable temperature (MDT) directly benefit from a low NET.

\section{Minimum Resolvable Temperature (MRT)}

The detector's sensitivity to temperature, or thermal signals, and the detector's ability to resolve (a relationship of physical size and thermal sensitivity) temperature differences are used to create an image that is displayed for the eye to see. From an image quality point of view, all of the design and 
manufacturing, signal processing, detector response uniformity, detector cooling, scanning mechanisms, etc., are manifest in a parameter called minimum resolvable temperature (MRT) difference. The MRT varies with spatial frequency (spacing) in a nonlinear manner; low MRT at low spatial frequency, and higher MRT at higher spatial frequencies.

Minimum Detectable Temperature (MDT)

An infrared sensor can perceive a signal much smaller than its angular resolution (detector subtense and MRT) and process the signal to provide the eye with a stimulus that is perceived, but not resolved.

Detection of an unresolved thermal signal is referred to as "hot spot" detection. One might compare an infrared sensor's parameters of MRT and MDT to the ability of one to resolve two stars in the sky to one's ability to see a star. One is able to see a star even though its angular subtense is much smaller than the eye can resolve, but one does not know if one is seeing one star, two, or a group - only that one sees something. The star or stars seen cannot be distinguished unless they are separated by an angular subtense greater than the eye's minimum resolution. The perception of "something" is not imagined.

\section{Documented Studies of Detection Ranges}

A literature survey identified several previous studies of detection ranges for night vision devices in maritime related scenarios. Several of these were related to Search and Rescue operations by the U.S. Coast Guard and the U.S. Army Aeromedical Research Laboratory. In particular, the Coast Guard studied the detection ranges for personnel in the water, 6 and 9 person rafts, and 18 foot and 21 foot boats. In addition, the Naval Research Laboratory (NRL) studied the detection ranges for combat rubber raiding craft (CRRC) and the Naval Postgraduate School studied the detection ranges from the Mark V Special Operations Craft. A summary of the results of these reports will be presented here. Only generalized results from the latter two will be presented due to security concerns.

\section{Coast Guard Studies}

One study (Plourde \& Marsee, 1991) ${ }^{20}$ studied the detection probability for unlighted 4 and 6 person life rafts in 3 to 5 foot seas and in 5.6 to 7.2 foot seas. Figures 53 and 54 present the detection probabilities for these conditions. From these figures, it can be seen that unlighted life rafts are difficult to detect at any range and become especially difficult at distances greater than $0.5 \mathrm{nmi}$ using GEN III night vision devices in $1 / 4$ moon to full moonlight. In this study, extraneous light from the search vehicles affected

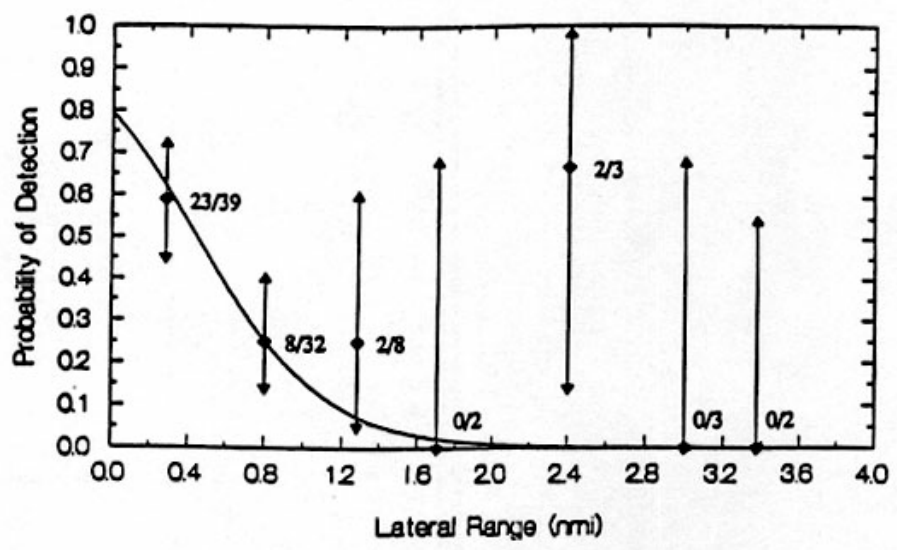

Fig. 53. Detection of unlighted life rafts (wave height between 3 and 5 feet). 
the performance of the night vision devices through "glare." Also, on dark nights, the GEN III devices yielded a "grainy" image in which it was difficult to detect the rafts much less identify them. Also, the presence of sea spray greatly reduced visibility.

A second study (Hover \& Plourde, 1990) ${ }^{21}$ studied the detection probability for GEN II and GEN III night vision devices on personnel in the water, 4 and 6 person life rafts, and 18 and 21 foot boats for three search conditions; (1) wave height of 1.3 to 2 feet, visibility 10 to $15 \mathrm{nmi}$ and no moon, (2) wave height 2.0 to 3.3 feet, visibility 6 to $15 \mathrm{nmi}$, and a visible moon, and (3) wave height 2.3 to 3.3 feet, visibility 6 to $15 \mathrm{nmi}$, and no moon. This study concluded that the detection performance of the night vision devices against the life raft was not more than 1/10 that of daylight performance. The night vision devices achieved only marginal detection performance against the personnel in the water. Even when targets passed close aboard (0 lateral range), only one-third were detected. Detection performance against 18 foot boats was approximately $1 / 10$ that of daylight performance and performance against 21 foot boats was approximately $1 / 4$ that of daylight performance. In addition, this study found that search platform

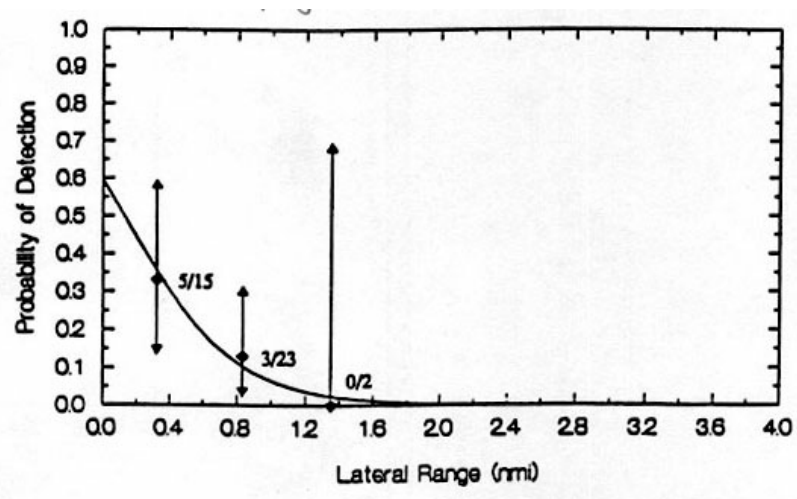

Fig. 54. Detection of unlighted life rafts (wave height between 5.6 and 7.2 feet).

motion, coupled with the narrow field of view, consistently causes seasickness and disorientation. Furthermore, the effectiveness of the night vision devices is inhibited by the constant presence of salt spray even when the users are sheltered. Light sources on the search platforms cause a great deal of interference with the night vision devices. This study concluded that searching for personnel in the water, small life rafts, and small boats with night vision devices were ineffective in seas greater than 3 feet. Figures 55 and 56 present the detection probability for personnel in the water and life rafts from helicopters in this study. Figures 57 and 58 present the detection probability for these same targets from 41 foot boats. Again in this study, extraneous light sources presented a glare problem as did low light levels for image intensifier operation, as well as sea spray. 


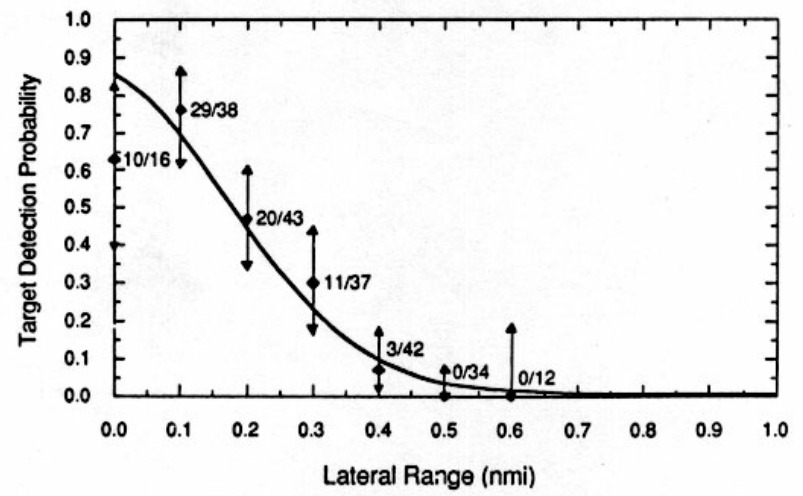

Fig. 55. Helicopter detection of personnel in the water (PIW) (visibility $=15 \mathrm{nmi})$.

The third study (Raunig \& Marsee, 1995) ${ }^{22}$ again used helicopter detection with GEN III night vision device for personnel in the water, 6 and 10 person life rafts, and 18 and 21 foot boats. This study againconcluded that personnel in the water targets are essentially invisible under these conditions. Figures 59 and 60 show the detection probability of small boats under visible moon and no moon conditions. From

these figures, it can be seen that small boats under visible moon conditions are detectable about $0.75 \mathrm{nmi}$ away and with no moon conditions are detectable about $0.5 \mathrm{nmi}$ away. Figures 61 and 62 show the detection probability of 6 and 10 person life rafts under visible moon and no moon conditions. From these figures, it can be seen that life rafts are generally detectable at over $2 \mathrm{nmi}$ distance under visible moon conditions, while under no moon conditions, they are only detectable at less than a mile distance. Figures 63 and 64 show the detection probability of personnel in the water under visible moon and no moon conditions. From these figures, it can be seen that there is very little detection probability for personnel in the water with visible moon and virtually no probability of detection with no moon.

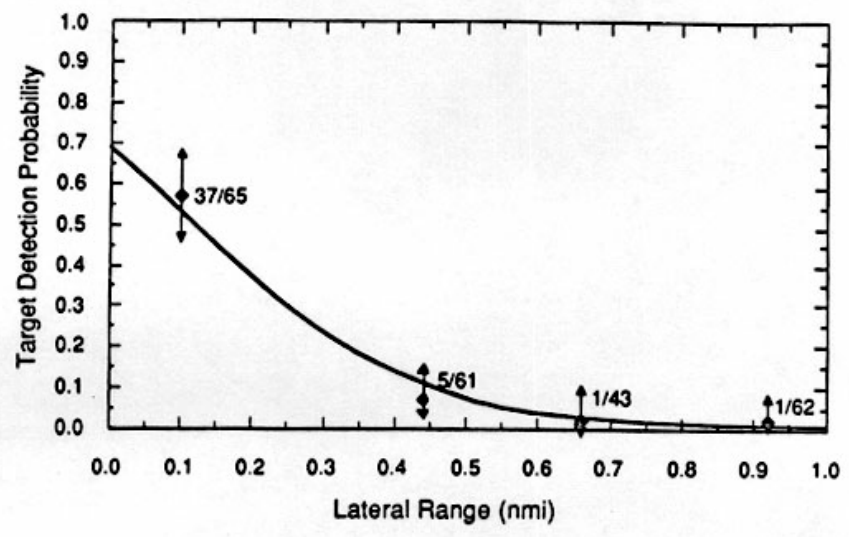

Fig. 56. Helicopter detection of life rafts. 


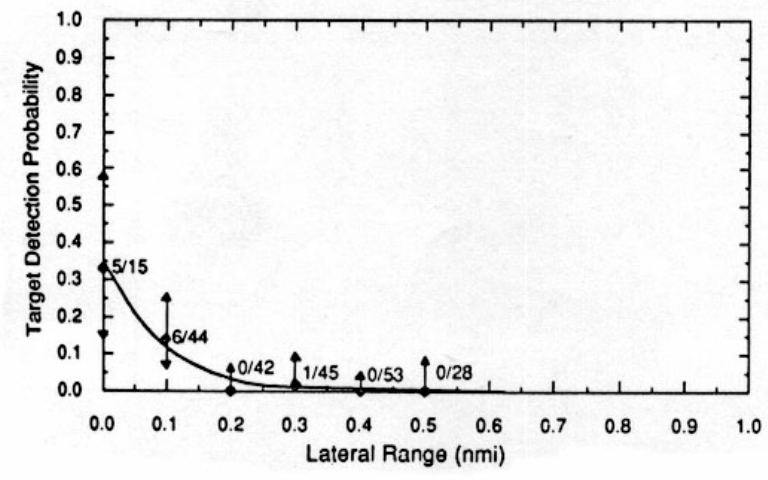

Fig. 57. Detection of PIWs by 41 foot boats.

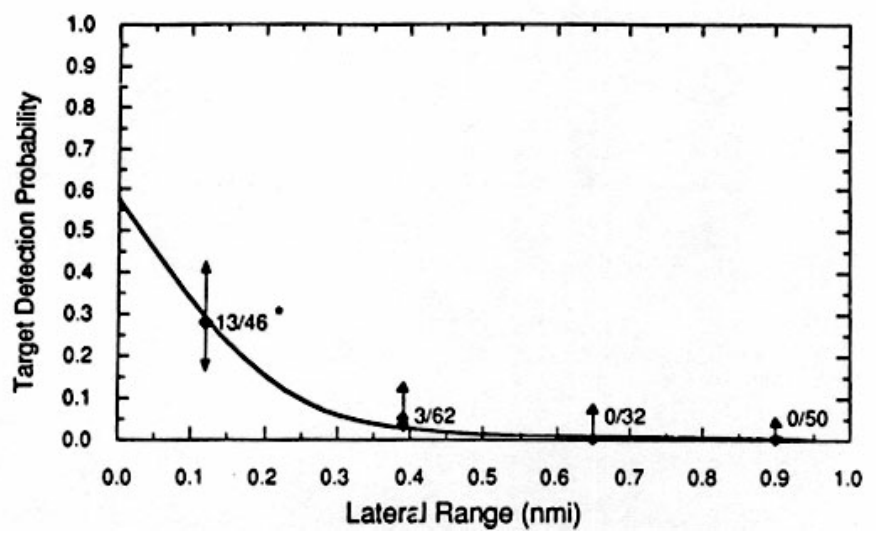

Fig. 58. Detection of life rafts from 41 foot boats.

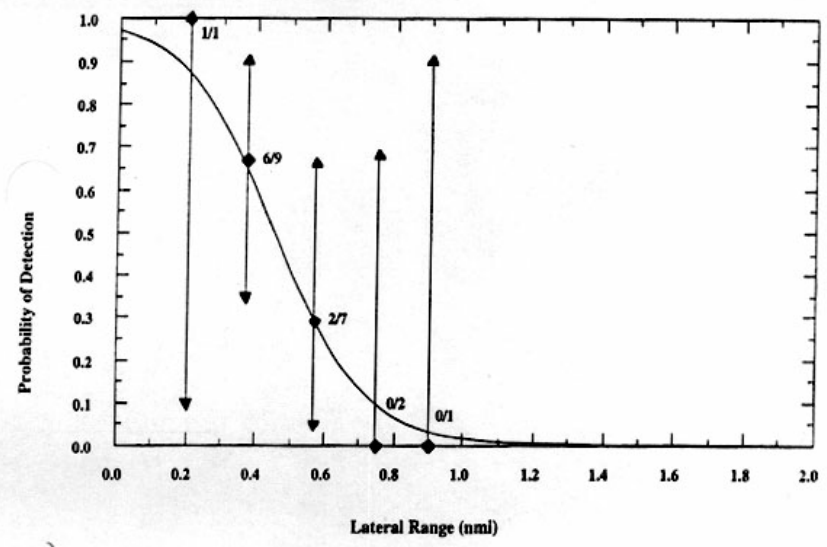

Fig. 59. Small boat night vision device detection, visible moon. 


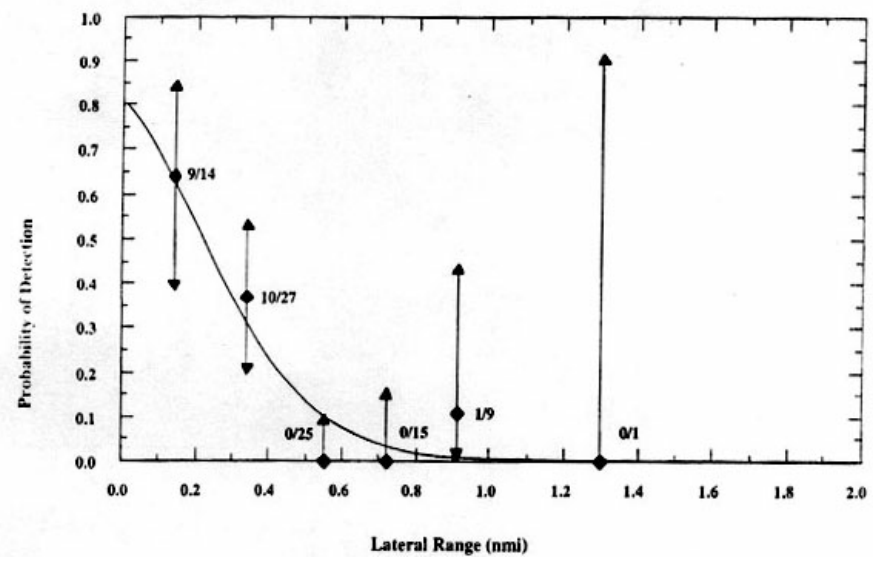

Fig. 60. Small boat night vision device detection, no moon.

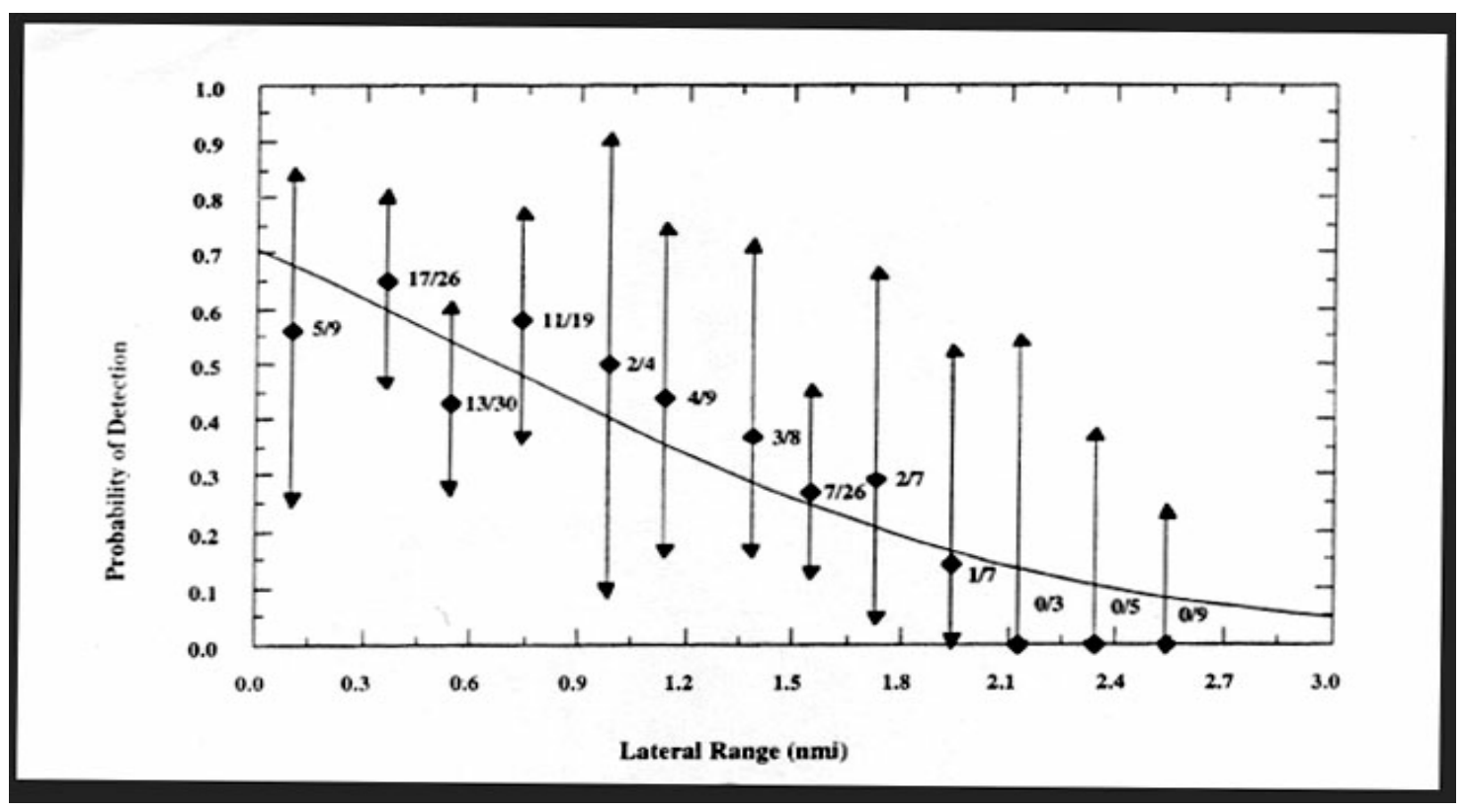

Fig. 61. Life-raft night vision device detection, visible moon. 


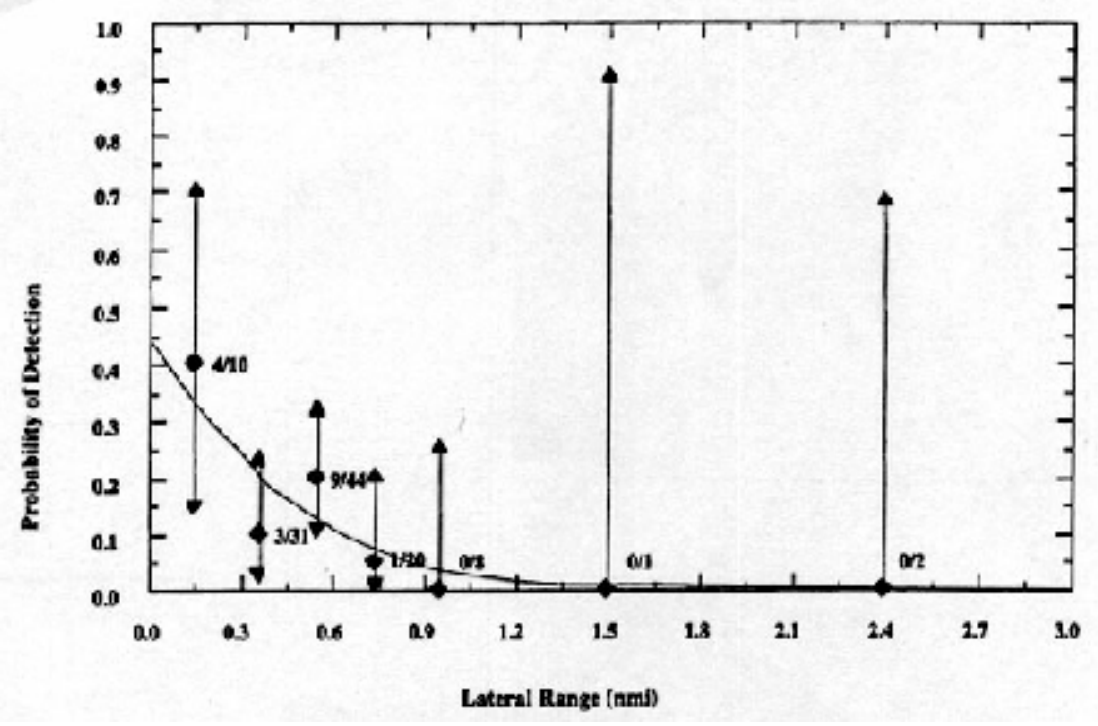

Fig. 62. Life-raft night vision device detection, no moon.

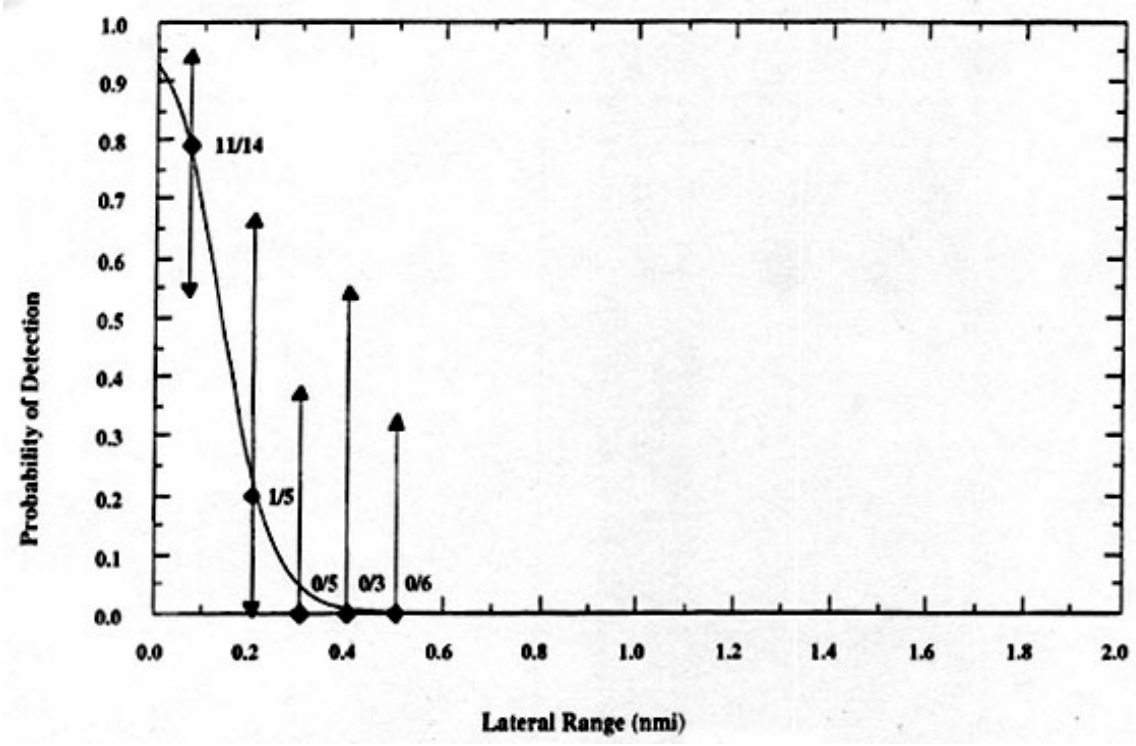

Fig. 63. Personnel in the water (PIW). Detection with night vision devices, visible moon.

The fourth study (Rabin, 1994) ${ }^{23}$ deals with infrared imager detection and recognition of human targets from helicopters on land in an uncluttered environment with two similar infrared imagers; Lockheed Martin's Pilot Night Vision System (PNVS) and FLIR Systems' Safire. Relevant characteristics of the two systems are show in Table 6. 


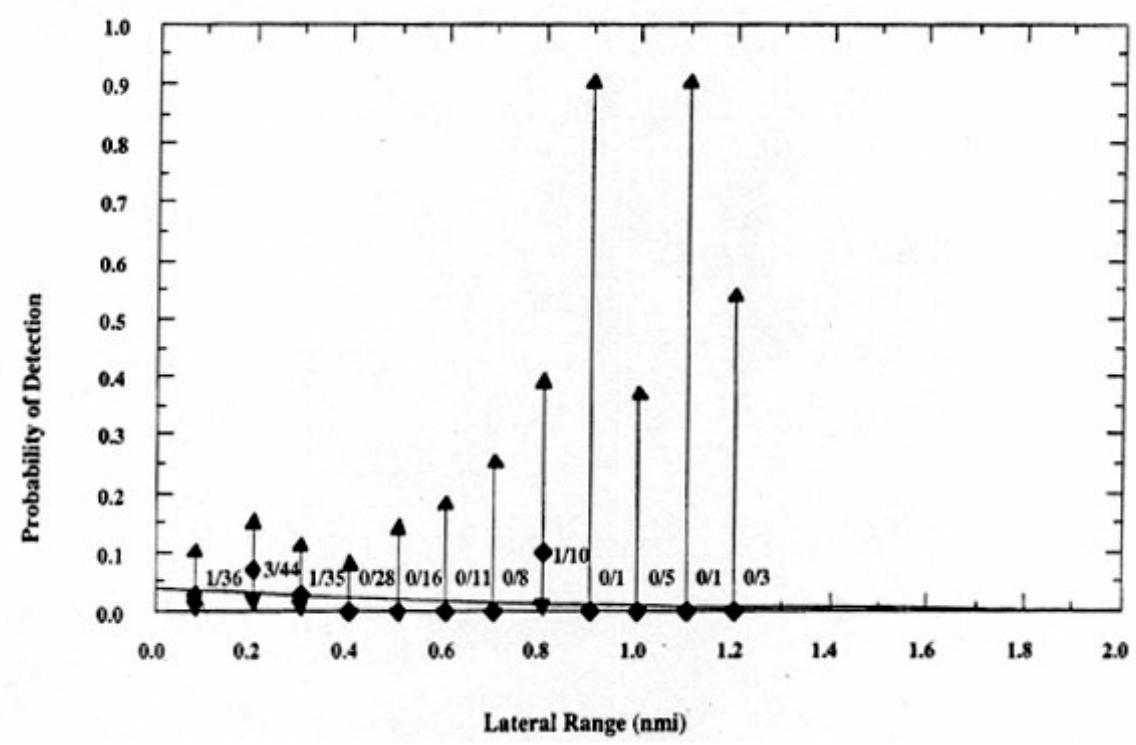

Fig. 64. Personnel in the water (PIW). Detection by night vision devices, no moon.

The study concludes, as shown in Figure 65, that detection occurred at about $1 \mathrm{~km}$ with each system. This distance is consistent with the infrared imagers' resolution, target contrast, and nature of the detection task. The human target subtended a vertical dimension of about $1.6 \mathrm{~m}$ which, at $1 \mathrm{~km}$, corresponds to a resolution of 20/110. While this resolution is less than that achieved with maximum contrast (20/60 to 20/70), it is likely that the target contrast, while high, was not maximum under the testing conditions, and thus resolution was somewhat reduced. In addition, the dynamic nature of the task and requirement to scan the whole field of view probably diminished the detection range relative to the value predicted from system resolution alone.

Table 6. Characteristics of Infrared Imager Systems in Rabin, 1994 Study

\begin{tabular}{|c|c|c|}
\hline System Characteristic & Safire & PNVS \\
\hline Spectral Sensitivity & $8-12$ microns & 8 - 12 microns \\
\hline Resolution & $\begin{array}{l}0.93 \text { milliradians } \\
(20 / 64)\end{array}$ & $\begin{array}{l}0.83 \text { milliradians } \\
(20 / 57)\end{array}$ \\
\hline Magnification & $1.86 \mathrm{X} \& 10.5 \mathrm{X}$ & $1 \mathrm{X}$ \\
\hline Field-of-View & $\begin{array}{l}\text { wide: } 16.8^{\circ} \times 28^{\circ} \\
\text { narrow: } \quad 3^{\circ} \times 5^{\circ}\end{array}$ & $30^{\circ} \mathrm{X} 40^{\circ}$ \\
\hline Field-of-Regard & $\begin{array}{l}\text { azimuth: } 360^{\circ} \\
\text { elevation: }+30^{\circ} \text { to }-120^{\circ}\end{array}$ & $\begin{array}{ll}\text { azimuth: } & +90^{\circ} \text { to }-90^{\circ} \\
\text { elevation: } & +45^{\circ} \text { to }-20^{\circ}\end{array}$ \\
\hline
\end{tabular}


Figure 66 presents the distance for recognition of the target as human. There was a significant difference between systems in the recognition distance because the Safire system was equipped with a $5 \mathrm{X}$ magnification lens. The $4 \mathrm{X}$ greater range for recognition with Safire reflects the magnification capability of this system. Once detection occurred, the users applied the 5X magnification to "zoom in" on the target and recognize relevant detail. The $4 \mathrm{X}$ greater range is consistent with magnification of approximately $5 \mathrm{X}$ since a small amount of time was required to focus the system and recognize the target (The user was flying in a helicopter toward the target).

\section{Office of Naval Research Study}

This study (Trusty \& Welch, 1995) ${ }^{24}$ investigated the detection limits for GEN II and GEN III night vision device, both US and Russian, against a combat rubber raiding craft (CRRC) shown in Figure 67. This study gave considerable insight into the detection problem.

Because of the possibility of bioluminescence aiding in the detection of a craft moving through the water, there exists many locations where it would be highly advantageous to use a sensor that is sensitive in the spectral range of 0.45 to $0.55 \mu \mathrm{m}$. GEN II devices, therefore, would probably be the sensors of choice for surveillance for littoral defense.

While infrared based night vision devices were not included in this study, an 8 to $12 \mu \mathrm{m}$ thermal imager was used to bore-sight the eye-safe laser rangefinder used. The study noted that under the majority of conditions, the thermal imaging device would readily outperform the visual systems and assure that the range to the target would always be known.

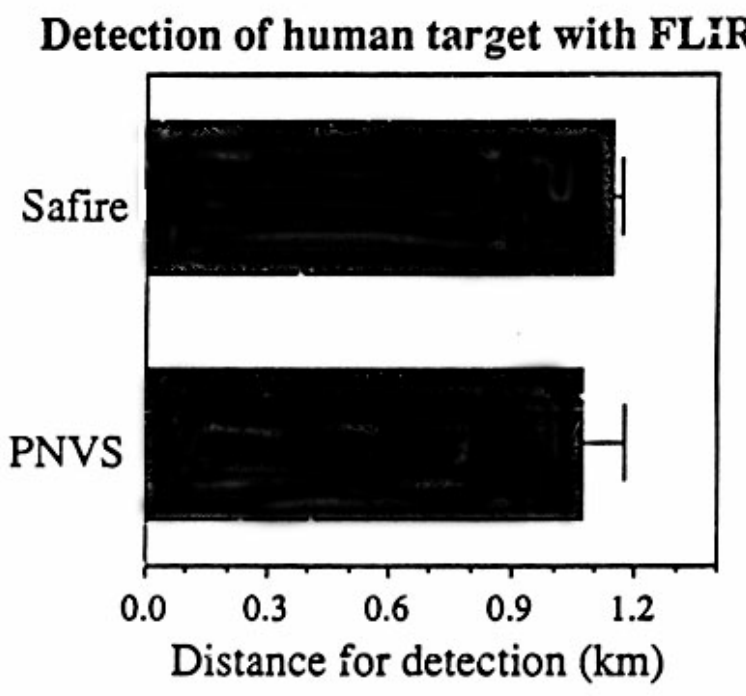

Fig. 65. Mean distance for detection of human targets by infrared imagers.

This study concluded that for the clear-sky front-lit case, the crew member in the hooded battle dress uniform (BDU) jacket in the rubber craft and the wake were normally the objects of detection as they were positive contrast against the darker water. A counter-example is where the raised bow of the craft hides the positive-contrast BDU, but the black craft body is still visible as a negative-contrast target. For the overcast night conditions, the black boat body was the object of detection as negative contrast against 
the lighter water. In general, when the target provided a positive contrast against the background, longer detection ranges were achieved.

This study also noted that the standard BDU, currently designed to match a foliage background with a reflectivity spectrally similar to chlorophyll, makes a very poor match to a sea background. The BDU colors spectrally reflect more in the wavelengths beyond $0.7 \mu \mathrm{m}$. This is what makes the cloth look dark in the night vision device. The spectral reflectivity of camouflage cloth versus GEN II, GEN III, and the eye's spectral sensitivity is illustrated in Figure 68.

The night vision devices studies are given in Table 7.

The boat was never observed in a condition of positive contrast. Therefore, the reflectivity of the boat (i.e., the rubber sponsor material) could be increased from the current $4 \%$ to $11 \%$ or $17 \%$. Figure 69 presents the spectral reflectivity of the rubber material of the combat rubber raiding craft (CRRC). Wake was a strong detection object and increased wind increases wake. Therefore, for rough water conditions, a slow approach with a resulting small wake is recommended. Also a true back-lit case, with the boat seen as a silhouette, is very easily seen.

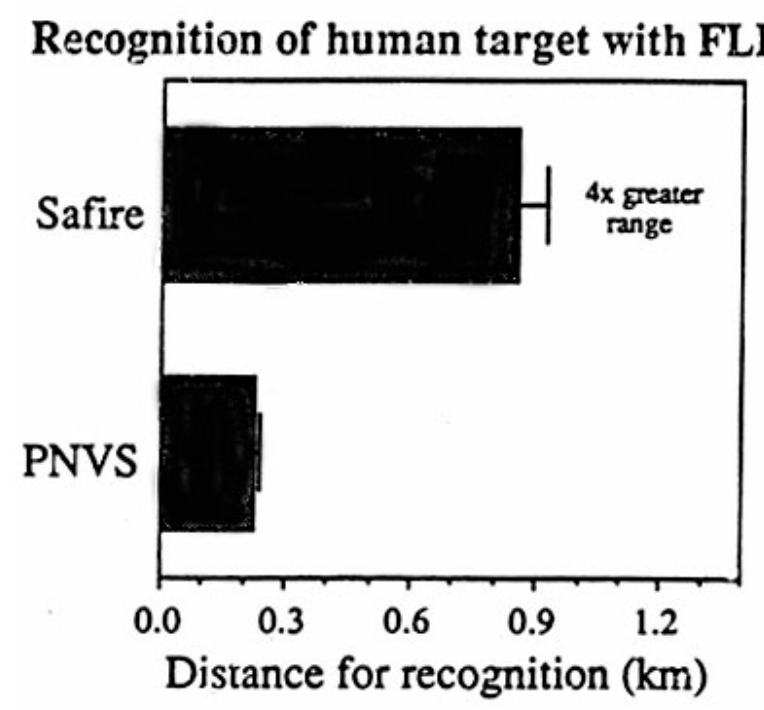

Fig. 66. Mean distance for recognition of the human target by infrared imagers.

The detection ranges from this study are classified. The reader is referenced to the original report for further information.

A second ONR study (Who \& Who, 1998) ( $^{5}$ also investigated the effects of the atmosphere and obscurants on the performance of night vision devices. Obscurants induced on the battlefield (e.g., tactical smokes, artillery-produced dust) are produced by localized sources. The source may be instantaneous (artillery bursts or explosions), continuous (smoke generators or smoke pots), or continuous and moving (dust behind a vehicle). The amount of obscurant along a line of sight can vary considerably in a very short time. Table 8 lists the effects of combat-induced obscurants. 


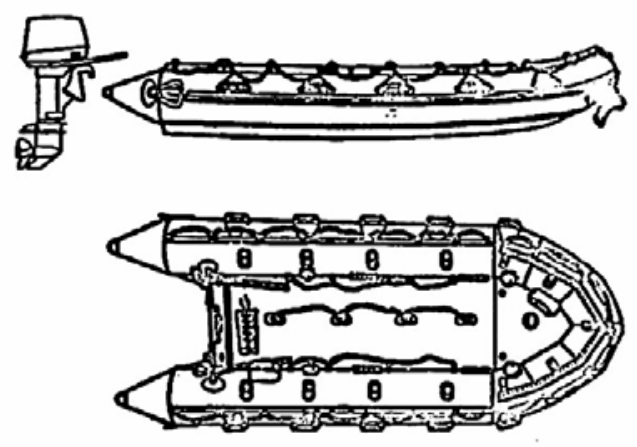

Fig. 67. (U) CRRC (Combat Rubber Raiding Craft).

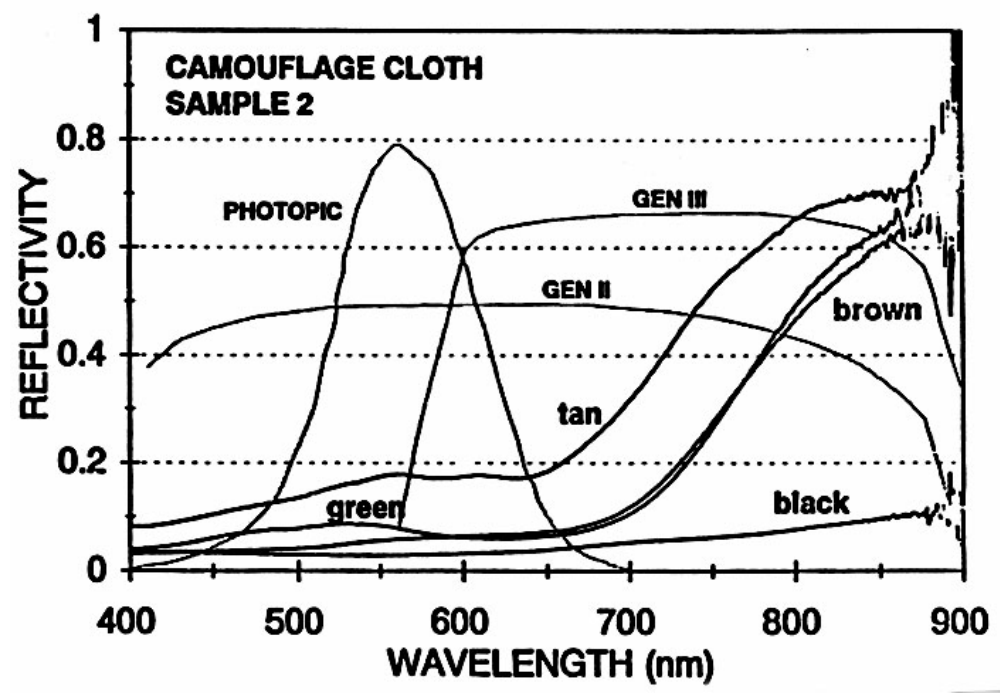

Fig. 68. (U) Reflectivity of a battle dress uniform cloth.

This study examined the effects of the environment through atmospheric transmittance on detection and imaging. A computer model (MODTRAN) was exercised to determine atmospheric transmittance for a horizontal path using representative atmospheric, aerosol, cloud, and rain models for various scenarios. The model was run to determine atmospheric transmittance in the visible, mid, and far IR wavelength band ( 0.35 to $0.9,3$ to 5 , and 8 to 12 micrometers, respectively) for varying ranges of $0.1,0.5,1,3,5$, and 10 kilometers. 
Table 7. Image Intensifier Based Night Vision Equipment for NRL Study

\begin{tabular}{|c|c|c|c|c|c|c|c|}
\hline $\begin{array}{l}\text { Night Vision } \\
\text { Device }\end{array}$ & Gen & FOV & Mag & Gain & Res & $\begin{array}{l}\text { Country } \\
\text { of Origin }\end{array}$ & Comments \\
\hline AN/PVS-7B & GEN III & $40^{\circ}$ & 1.0 & $27 \mathrm{~K}$ & $\begin{array}{c}45 \\
1 \mathrm{p} / \mathrm{mm}\end{array}$ & US & Similar to M972 \\
\hline AN-PVS-5C & GEN II & & 1.0 & $12 \mathrm{~K}$ & $\begin{array}{c}32 \\
1 \mathrm{p} / \mathrm{mm}\end{array}$ & US & \\
\hline M972-002 & GEN III & $40^{\circ}$ & 1.0 & $27 \mathrm{~K}$ & $\begin{array}{c}45 \\
1 \mathrm{p} / \mathrm{mm}\end{array}$ & US & $\begin{array}{c}\text { Similar to PVS- } \\
\text { 7B }\end{array}$ \\
\hline AN/PVS-4 & GEN II & & 3.7 & $50 \mathrm{~K}$ & $\begin{array}{c}32 \\
1 \mathrm{p} / \mathrm{mm}\end{array}$ & US & Spotting Scope \\
\hline MPN30K & GEN II & $15^{\circ}$ & 2.5 & $30 \mathrm{~K}$ & $\begin{array}{c}25 \\
1 \mathrm{p} / \mathrm{mm}\end{array}$ & Russian & Binocular \\
\hline MPN95K & GEN II & $10^{\circ}$ & 3.4 & $\begin{array}{l}60 \mathrm{~K}- \\
95 \mathrm{~K}\end{array}$ & $\begin{array}{c}25 \\
\mathrm{lp} / \mathrm{mm}\end{array}$ & Russian & Rifle Scope \\
\hline Moonwalker & GEN II & $20^{\circ}$ & 1.0 & $1 \mathrm{~K}$ & $\begin{array}{c}20 \\
\mathrm{lp} / \mathrm{mm}\end{array}$ & Russian & $\begin{array}{l}\text { Monocular } \\
\text { Very Poor } \\
\text { Quality }\end{array}$ \\
\hline MPN60K & GEN II & $12^{\circ}$ & 5.2 & $\begin{array}{l}35 \mathrm{~K}- \\
60 \mathrm{~K}\end{array}$ & $\begin{array}{c}25 \\
1 \mathrm{p} / \mathrm{mm}\end{array}$ & Russian & Spotting Scope \\
\hline
\end{tabular}

SPONSON MATERIAL

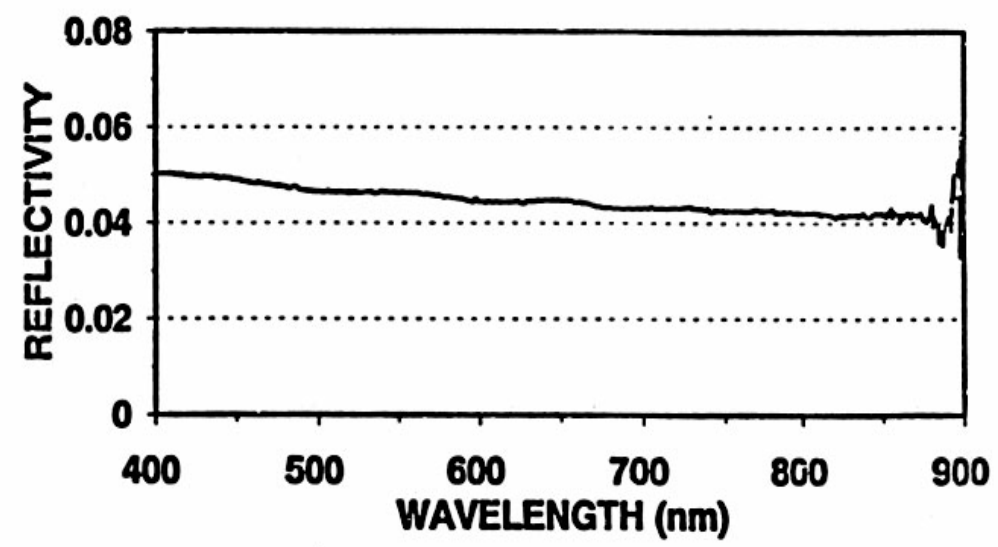

Fig. 69. (U) Reflectivity of a CRRC sponson material sample. 
During summer weather, the study concluded that for ranges beyond 1 kilometer, the transmittance degrades especially in the visible window region where it falls below $50 \%$. The visible window region has fewer anomalies than both of the infrared windows. In the mid IR window, the region between approximately 4.2 and 4.3 microns shows substantial molecular absorption where the transmittance falls nearly to zero.

During the summer season with fog present, virtually no transmittance exists in both the visible and mid IR window regions for range beyond 1 kilometer. The far IR window region shows greater transmittance than in the visible region, while the visible region shows greater transmittance than in the mid IR region.

During the summer season with both fog and rain, no transmittance exists for any of the window regions for ranges greater than 1 kilometer. For ranges of 0.5 to 1 kilometer, the transmittance falls to $20 \%$ or less in all of the window regions. With heavy fog only, the far IR region shows greater transmittance than in the visible, and the visible shows greater transmittance than in the mid IR region.

During the winter season, the atmospheric transmittance is nearly identical to that of the summer season. The mid IR window shows slightly greater transmittance than the corresponding summer plots. In the far IR window, transmittance is much greater in the winter than the summer especially for the longer ranges $(>1 \mathrm{~km})$.

For a tropical atmosphere with the presence of very heavy rain, the transmittance is nearly zero for all the window regions for ranges greater than 1 kilometer. Transmittance in the visible window region appears slightly higher than in the IR window regions.

Environmental factors influence the performance of electro-optic sensors principally by reducing transmittance. Environment factors also affect sensor performance by altering the target contrast and ambient light level. In addition, environmental factors influence the effectiveness of combat-induced obscurants. Wind and atmospheric stability affect the placement, motion, and dispersion of smoke and chemical clouds. Because the environment influences the performance of sensors, selection of the appropriate night vision device in performing a mission requires great consideration of the type of environment the equipment will be required to operate in. For example, longer wavelength systems offer advantages over visual ones in fog. Filters, contrast enhancement, and image intensification can help to offset some of the problems caused by haze.

Naval Postgraduate School's study is classified and represents the computer modeling of the detection limits of the Mark V Special Operations Craft. The reader is referenced to the original report for further information. 
Table 8. Effects of Combat Induced Obscurants

\begin{tabular}{|c|c|c|}
\hline Obscurant & Visible and Near IR & Infrared \\
\hline \multicolumn{3}{|l|}{ Smokes } \\
\hline Phosphorus & Severe & Severe - Moderate \\
\hline Hexacholoroethane & Severe & Moderate - Negligible \\
\hline Oil-Based & Severe & Negligible \\
\hline \multicolumn{3}{|l|}{ Dust } \\
\hline Artillery & Severe - Moderate & Severe - Moderate \\
\hline Vehicular & Severe - Moderate & Moderate - Negligible \\
\hline Helicopter & Severe - Moderate & Moderate - Negligible \\
\hline \multicolumn{3}{|l|}{ Explosions } \\
\hline Debris & Moderate - Time Transient & Moderate - Negligible - Time Transient \\
\hline Heat & Negligible & $\begin{array}{c}\text { Severe - Moderate - Time Transient - } \\
\text { Area Limited }\end{array}$ \\
\hline \multicolumn{3}{|l|}{ Fire } \\
\hline Particulates & Severe - Moderate & Severe - Negligible \\
\hline Gaseous & Negligible & Severe - Negligible \\
\hline Turbulence & $\begin{array}{c}\text { Severe - Moderate - Negligible - Area } \\
\text { Limited }\end{array}$ & Moderate - Negligible - Area Limited \\
\hline \multicolumn{3}{|l|}{ Launcher } \\
\hline Muzzle Flash & $\begin{array}{l}\text { Severe - Moderate - Negligible - Time } \\
\text { Transient - Area Limited }\end{array}$ & re - Moderate - Area Limited \\
\hline Rocket Plume & Severe - Moderate - Negligible & Severe - Negligible \\
\hline Dust & Severe - Moderate - Negligible & Severe - Negligible \\
\hline
\end{tabular}




\section{REFERENCES}

1. R. E. Miller and T. J. Tredici, Night Vision Manual for the Flight Surgeon, AL-SR-1992-0002, Armstrong Laboratory, Brooks Air Force Base, TX, August 1992.

2. P. H. Orell, "Application of Image Intensifier Technology to the Military, Scientific, Industrial, Educational, and Medical Communities," Masters thesis, University of Wisconsin-Stout, Menomonie, WI, May 1980.

3. M. L. Vatsia, Atmospheric Optical Environment, ECOM-7023, Defense Technical Information Center, Fort Monmouth, NJ, September 1972.

4. A. Berry, W. Perez, J. Houchard, E. Stellini, and R. Sanchez, Tactical Night Vision System Operational and Functional Requirements Analysis, HSD-TR-1991-0022, Science Applications International Corporation, Dayton, OH, April 1994.

5. $\quad$ Classified Report, "Preliminary Special Reconnaissance Mission Analysis," November 1994.

6. J. C. Kotulak and C. E. Rash, Visual Acuity with Second and Third Generation Night Vision Goggles Obtained from a New Method of Night Sky Simulation Across a Wide Range of Target Contrast, USAARL Repot No. 92-9, United States Army Aeromedical Research Laboratory, Fort Rucker, Al, January 1992.

7. R. E. Miller, W. F. Provines, M. G. Block, J. W. Miller, and T. J. Tredici, Comparative Visual Performance with ANVIS and AN/PVS-5A night Vision Goggles Under Starlight Conditions, USAFSAM-TR-84-28, USAF School of Aerospace Medicine, Brooks Air Force Base, TX, August 1984.

8. R. W. Wiley, Visual Acuity and Stereopsis with Night Vision Goggles, USAARL Report No. 89-9, United States Army Aeromedical Research Laboratory, Fort Rucker, AL, June 1989.

9. E. J. Bender, C. E. Bradford, and R. J. Stefanik, Comparison of Second and Third Generation Night Vision Goggles in Time-Limited Scenarios, AMSEL-NV-TR-0080, Center for Night Vision and Electro-Optics, Fort Belvoir, VA, July 1991.

10. J. T. Riegler, J. D. Whiteley, H. L. Task, and J. Schueren, The Effect of Signal-To-Noise Ratio on Visual Acuity Through Night Vision Goggles, AL-TR-1991-0011, Air Force Systems Command, Wright-Patterson Air Force Base, OH, March 1991.

11. H. K. Pollehn, Analysis of Field Tests Comparing Second and Third Generation Image Intensifiers, AMSEL-NV-TR-0069, Center for Night Vision and Electro-Optics, Fort Belvoir, VA, October 1988.

12. R. J. Beaton, "A Human Performance Based Evaluation of Quality Metrics for Hard-Copy and Soft-Copy Digital Imaging Systems," doctoral dissertation, Virginia Polytechnic Institute and State University, Blacksburg, VA, 1984.

13. D. H. Abel, An Image Quality Analysis of ANVIS-6 Night Vision Goggles, Air Force Institute of Technology, Wright-Patterson Air Force Base, OH, December 1994.

14. D. E. Schmieder, M. R. Weathersby, W. M. Finlay, and T. J. Doll, Clutter and Resolution Effects on Observer Static Detection Performance, AFWAL-TR-82-1059, Georgia Tech Research Institute, 1982.

15. M. R. Snapp, M .J. Gouveia, and P. F. Hilton, Impact of Scene Shadows on Target Acquisition, CR-920132-1, U. S. Army Atmospheric Sciences Laboratory, White Sands Missile Range, NM, August 1992.

16. L. M. Biberman and E. A. Alluisi, Pilot Errors Involving Head Up Displays (HUDs), Helmet Mounted Displays (HMDs) and Night Vision Goggles (NVGs), IDA Paper P-2638, Institute for Defense Analysis, Alexandria, VA, 1992.

17. J. J. Armentrout, "An Investigation of Stereopsis with AN/ANVIS-6 Night Vision Goggles at Varying Levels of Illuminance and Contrast," unpublished Master's Thesis, Virginia Polytechnic Institute and State University, Blacksburg, VA, 1993.

18. H. L. Snyder, Flat Panel Displays and CRT's, pp. 71-90, Image Quality: Measures and Visual Performance, ed. L. E. Tannas, New York: Van Nostrand Reinhold, 1985.

19. J. M. Lloyd, Thermal Imaging Systems.

20. R. Q. Robe, J. V. Plourde, and R. L. Marsee, Evaluation of Night Vision Goggles (NVG) for Maritime Search and Rescue, CG-D-13-92, U. S. Department of Transportation -United States Coast Guard, Washington, DC, November 1991.

21. W. H. E. Reynolds, R. Q. Robe, G. L. Hover, and J. V. Plourde, Evaluation of Night Vision Goggles for Maritime Search and Rescue, CG-D-14-90, U. S. Department of Transportation-United States Coast Guard, Washington, DC, April 1990. 
22. T. E. McClay, R. Q. Robe, D. L. Raunig, and R. L. Marsee, Evaluation of Night Vision Goggles $(N V G) /$ Laser Illuminator for Maritime Search and Rescue, CG-D-36-95, U. S. Department of Transportation-United States Coast Guard, Washington, DC, May 1995.

23. J. Rabin, Forward-Looking Infrared: Capabilities for Search and Rescue, USAARL Report No. 94-11, United States Army Aeromedical Research Laboratory, Fort Rucker, AL, February 1994.

24. G. L. Trusty and J. A. Welch, Night Vision Measurements of a Combat Rubber Raiding Craft (U), NRL/MR/5630-95-7783, Naval Research Laboratory, Washington, DC, September 1995. 


\section{APPENDIX A}

\section{Currently Fielded Image Intensifier Based Night Vision Devices}

\begin{tabular}{|c|c|}
\hline AN/PVS-7B (M962/M963) & \\
\hline $\begin{array}{l}\text { The AN/PVS-7B is a single tul } \\
\text { of the U.S. Army. The M962/I } \\
\text { worn around the neck by a stra } \\
\text { with a low battery warning sen } \\
\text { the scene for map reading, nigh } \\
\text { used in conjunction with a pers } \\
\text { This allows for IR aim dot plac } \\
\text { M962/M963 can be focused wi } \\
\text { be field adapted to accept } 3 \mathrm{x} \text { ar } \\
\text { a high strength plastic polymer } \\
\text { approximately } 16 \mathrm{~cm} \text {. }\end{array}$ & $\begin{array}{l}\text { designed for general land use and is the primary NVG } \\
\text { facemask or flip-up helmet mount. The system can be } \\
\text { ggle is powered by two "AA" batteries and is equipped } \\
\text { a built-in infrared LED designed to artificially illuminate } \\
\text { nance work, and other applications. The goggle may be } \\
\text { ed with a boresighted infrared LASER aiming device. } \\
\text { th aiming through a scope or traditional iron sights. The } \\
\text {-issue Arctic mittens. In addition, this system can easily } \\
\text { anced magnification. The M963/M963 is constructed of } \\
\text { nclude a length of approximately } 15 \mathrm{~cm} \text { and a width of }\end{array}$ \\
\hline AN/PVS-7A/C (M972/M973) & \\
\hline $\begin{array}{l}\text { The M972/M973 are night visi } \\
\text { lightweight, compact, single tu } \\
\text { specifications and can be used } \\
\text { highly effective for short to me } \\
\text { emitting diode (LED) provides } \\
\text { objective lens as desired. Typi } \\
\text { enforcement, search and recuse } \\
\text { improved version is modified t } \\
\text { to depths of } 20 \text { M.. It is constru } \\
15 \mathrm{~cm} \text { by } 13 \mathrm{~cm} \text {. }\end{array}$ & $\begin{array}{l}\text { r high resolution, image intensified night vision in a } \\
\text { his rugged, water-resistant NVG is designed to military } \\
\text { s a hand-held viewer. The standard } 1 X \text { magnification is } \\
\text { n totally dark environments, an internal infrared light- } \\
\text { n this system can be substituted with a } 4 \mathrm{X} \text { or } 6 \mathrm{x} \\
\text { vert surveillance, combat, security uses, law } \\
\text { g control, wildlife observation, and navigation. An } \\
\text { ty requirements for the U.S. Navy. It can be employed } \\
\text { gth plastic alloy. Length and width are approximately }\end{array}$ \\
\hline Weight: $\quad 1.5$ pounds & $\begin{array}{ll}\text { Power Requirements: } & \begin{array}{l}\text { BA5567 Li Battery } \\
(2) \text { BA 3058 "AA" }\end{array} \\
\end{array}$ \\
\hline Range: $\quad 200-350$ yards & $\begin{array}{lll}\text { Battery Life: } & \text { BA } 5567 & 12 \text { hours @ 23C } \\
& \text { BA 3058 } & 40 \text { hours @23C }\end{array}$ \\
\hline Magnification: $1 \mathrm{X}$ & Tube Type: $\quad 18 \mathrm{~mm}$ GEN III \\
\hline Field of View: $\quad 40$ Degrees & \\
\hline
\end{tabular}




\begin{tabular}{|c|c|}
\hline \multicolumn{2}{|c|}{ AB/PVS-5B (M912A/M915A) } \\
\hline \multicolumn{2}{|c|}{$\begin{array}{l}\text { The M912A and M915A Night Vision Goggles are completely self-contained night vision systems providing } \\
\text { passive viewing capability under moonlit or starlit conditions. These systems are improved versions of the } \\
\text { AN/PVS-5A. Both the M912A and M915A operate on two "AA" batteries. These improved models incorporate } \\
\text { a higher output near-infrared source for situations where there is no light or extremely low light. It can be } \\
\text { switched on whenever needed for map reading, vehicle repair, dark building or bunker ingress, and other viewing } \\
\text { tasks. The systems employ two Generation II image intensifier tube assemblies. The improved flash performance } \\
\text { and extended dynamic range of the M915A allow its use under field conditions previously too harsh for night } \\
\text { vision applications. The systems are constructed of high tensile strength metals with a composite facemask } \\
\text { assembly. }\end{array}$} \\
\hline Weight: 2.2 pounds & $\begin{array}{ll}\text { Power Requirements: } & \begin{array}{l}\text { BA5567 Li Battery } \\
\text { BA3058 "AA" }\end{array} \\
\end{array}$ \\
\hline Range: $\quad 200-300$ yards & $\begin{array}{ll}\text { Battery Life: } & \text { BA 5567 - 12 hours @ 23C } \\
& \text { BA 3058-40 hours @ 23C } \\
\end{array}$ \\
\hline Magnification: $1 \mathrm{X}$ & Tube Type: $18 \mathrm{~mm}$ GEN II \\
\hline Field of View: 40 degree & \\
\hline
\end{tabular}

\begin{tabular}{|c|c|}
\hline \multicolumn{2}{|l|}{ AN/AVS-6 } \\
\hline \multicolumn{2}{|c|}{$\begin{array}{l}\text { The Aviator's Night Vision Imaging System (ANVIS) goggles provide improved night vision using available light } \\
\text { from the night sky to enable the user to pilot rotary wing aircraft or to drive high speed ground vehicles. The } \\
\text { ANVIS has dramatically extended the operational mission capability of helicopter crews. Pilots can now carry out } \\
\text { search and rescue (SAR) along with nap-of-the-earth flights under moonlight or starlight. The primary features } \\
\text { include: MIL-spec design and construction, normal peripheral vision, comfortable } 25 \mathrm{~mm} \text { eye relief, low fatigue } \\
\text { design, flip-up stowage when not in use, superior light sensitivity and quality, fast flash response, simple } \\
\text { adjustments, fast critical situation breakaway, easy upgrade potential, and multiple system redundance for safety. } \\
\text { The system is designed to be full compatible with the U.S. Army aviator's helmet and can be modified to fit } \\
\text { foreign aviator helmets. It is designed to satisfy the mission requirements for covert night combat, border patrol } \\
\text { engagement and support, troop transport, reconnaissance, minesweeping, and navigation. }\end{array}$} \\
\hline Weight: 1.3 pounds & Power Requirements: (2) BA5567 Li Battery \\
\hline Range: $\quad 200$ feet AGL and below & Battery Life: 12 hours @ 23C \\
\hline Magnification: $1 \mathrm{X}$ & Tube Type: $18 \mathrm{~mm}$ GEN III \\
\hline Field of View: 40 degrees & \\
\hline
\end{tabular}




\begin{tabular}{|l|l|}
\hline AN/PVS-15 (M921) [Submersible] \\
\hline $\begin{array}{l}\text { The AN/PVS-15 is the next generation of light weight, self-contained twin tube goggle system developed for the } \\
\text { U.S. Navy. The submersible binocular night vision system (BNVS) twin tube design offers a better depth } \\
\text { perception than single tube systems and operational submersibility down to } 20 \mathrm{~m} \text {. This system maintains the same } \\
\text { performance characteristics found in the AN/AVS-6 ANVIS. Typical applications include special operations, } \\
\text { amphibious landing, fast attack craft (FAC) missions, and underwater operations. The AN/.PVS-15 can be } \\
\text { operated in a handheld mode or mounted on a facemask, headstrap, or helmet. A flip-up stowage position is } \\
\text { offered for user convenience. The submersible BNVS is particularly suited for operations where depth perception } \\
\text { is critical for mission performance such as controlling land vehicles and boats at high speed, waist gunner } \\
\text { accuracy on helicopters, open turret command or armored vehicles, and carrier deck operations at night. }\end{array}$ \\
\hline $\begin{array}{l}\text { Weight: 4.6 pounds } \\
\text { Rower Requirements: BA 1567 Mercury } \\
\text { BA 5567 Li }\end{array}$ \\
\hline Range: 300 - 500 yards & Battery Life: 12 hours @ 23C \\
\hline Magnification: $3 \mathrm{X}$ & Tube Type: 18mm GEN II+ or GEN III \\
\hline Field of View: 13 degrees & \\
\hline
\end{tabular}

\begin{tabular}{|c|c|}
\hline Aquila & \\
\hline \multicolumn{2}{|c|}{$\begin{array}{l}\text { The AQUILA III is an upgraded design of the low profile AQUILA I night vision weapons sight that now include } \\
\text { 4x or 6x objective with ballistic controls suitable for matching with individual, crew served, and sniper weapons. } \\
\text { Because AQUILA II has a straight through design, it offers excellent cheek-weld characteristics on the lower } \\
\text { carbine profile weapons with flat mounting rails in the } 5.56 \mathrm{~mm} \text { caliber family. The AQUILA III is constructed } \\
\text { from a proven, high-strength aluminum alloy designed to survive rugged military use and harsh environments. } \\
\text { The AQUILA III is designed to mount on a wide range of weapons incorporating STANAG or Weaver mounts. } \\
\text { Objective lens magnifications ranges form } 4 \mathrm{x} \text { to } 6 \mathrm{x} \text { depending on lens selected with a focus range of } 25 \mathrm{M} \text { to } \\
\text { infinity. Mechanical dimension are characterized by a length of } 26 \mathrm{~cm} \text { for the } 4 \mathrm{x} \text { version ro } 32 \mathrm{~cm} \text { for the } 6 \mathrm{x} \\
\text { version. Width ranges form } 9 \mathrm{~cm} \text { to } 11 \mathrm{~cm} \text { depending upon objective lens. Weight approximates } 1.3 \mathrm{~kg} \text { for the } 4 \mathrm{x} \\
\text { version and } 1.8 \mathrm{~kg} \text { for the } 6 \mathrm{x} \text { version. }\end{array}$} \\
\hline Weight: $\quad 2.7$ pounds & Power Requirements: (2) BA3058 “AA” \\
\hline Range: $\quad 400-800$ yards & Battery Life: 40 hours @ 23C \\
\hline Magnification: $4 \mathrm{X}$ & Tube Type: $\quad 18 \mathrm{~mm}$ Gen II+, SupreGen, or GEN III \\
\hline Field of View: 8.3 degrees & \\
\hline
\end{tabular}




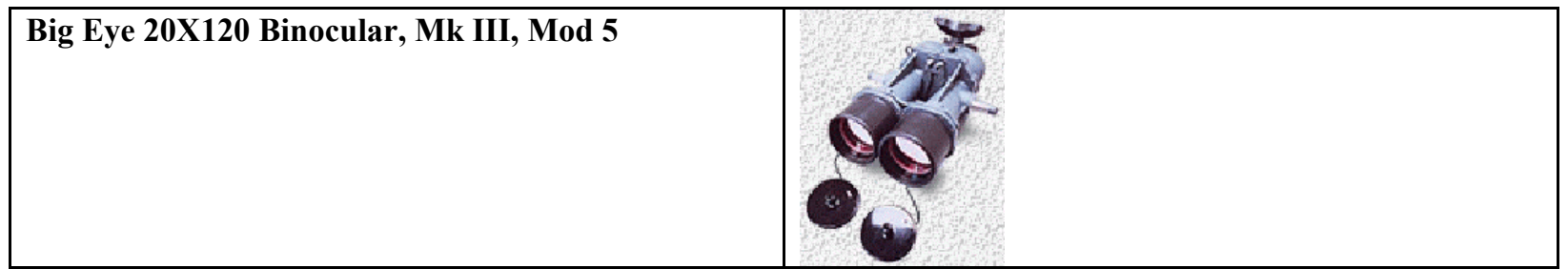

Big Eye is the U.S. Navy Standard 20X120 Binocular with a 70 degree field of view. The binocular is mounted on a height-adjustable carriage assembly and is adjustable through 70 degree elevation ranging from -10 degrees depression through +60 degrees elevation with reference to the horizon. It is free to rotate through a complete 360 degree in azimuth. Variable density polarizing filters may be introduced to reduce glare. Optical elements have an anti-reflective coating to increase light transmission. Controls of interpupillary adjustment and focusing are easily accessible and provision have been made to install an illuminated reticule if required.. Internal optics magnify objects $20 \mathrm{x}$. Eyeguards are provided to exclude light from escaping from around the observer's eyes. Focusing knobs enable the eyepieces to be individually adjusted. Big Eye can be mounted on MkIII Mod 1 through Mod 4 carriage assemblies without any accessories or additional hardware. The binocular assembly weights approximately $22.7 \mathrm{~kg}$ with the carriage assembly. Mechanical dimensions include a length of $52 \mathrm{~cm}$.

\begin{tabular}{|l|}
\hline AN/PVS-8 Long Range Observation Device \\
The AN/PVS-8 is a new generation long-range image intensified night vision system suitable for a \\
broad spectrum of night observation missions. Designed to meet new U.S. Navy requirements for \\
replacing current Generation I systems, it can be configured with either a Generation II or Generation \\
III image intensifier tube assembly. U.S. Navy systems are combined with a binocular eyepiece. An \\
optional monocular eyepiece is also available. A special facial eyeguard protects against back-glow. \\
All versions are easily adaptable for use with $35 \mathrm{~mm}$ still cameras or video cameras by removing the \\
eyepiece and installing an interface relay lens. Navy applications mount the system to a permanent \\
shipboard yolk trunnion, pintle fixture. Optional mounting systems, such as tripod legs, are available. \\
The system is powered by "AA" batteries and has both an automatic brightness control feature and \\
variable gain control. A flash protection circuit allows the tube to recover rapidly in time for an \\
observer to view a directed round hit targets. Magnification for the binocular system is $5 x$ whereas the \\
monocular system has a magnification of $11.6 x$. Mechanical characteristics include a maximum length \\
of $64 \mathrm{~cm}$ and an height of $47 \mathrm{~cm}$. It weighs in the range of $16 \mathrm{~kg}$.
\end{tabular}




\begin{tabular}{|l|}
\hline AN/TVS-5 Crew-Served Weapons Sight \\
\hline $\begin{array}{l}\text { The AN/TVS-5 system has been developed to accommodate changes in the night vision industry allowing for a } \\
\text { Generation III retrofit image intensifier tube assembly. It is suitable for a wide variety of night vision weapons } \\
\text { applications including heavy and medium machine guns, recoilless rifles, small cannons, and missile launchers. } \\
\text { Boresighting of the weapons system is accomplished by adjusting the azimuth and elevation actuators. A } \\
\text { shuttered rubber eyeguard protects the user from weapons recoil and prevents light from escaping from the } \\
\text { eyepiece. System magnification is } 6.2 x \text { with a focus range from } 25 \mathrm{~m} \text { to infinity. Mechanical dimensions are } \\
\text { characterized by a length of } 31 \mathrm{~cm} \text {, a height of } 17 \mathrm{~cm} \text { and a weight of approximately } 3 \mathrm{~kg} \text {. }\end{array}$ \\
\hline
\end{tabular}

\begin{tabular}{|l|}
\hline AN/PVS-4 U.S. Army Night Sight \\
\hline $\begin{array}{l}\text { The AN/PVS Night vision Individual Served Weapons Sight has continued to receive system improvements over } \\
\text { its lifetime which have resulted in a Generation III image intensifier assembly retrofit capability. It is suitable for } \\
\text { a wide variety of night vision weapon applications including } 5.56 \mathrm{~mm} \text { and } 7.62 \mathrm{~mm} \text { rifles, light and medium } \\
\text { machine guns, and racket and grenade launchers. The image intensifier tube assembly features automatic } \\
\text { brightness and gain control. Boresighting of the weapon system is accomplished by adjusting the azimuth and } \\
\text { elevation actuators. A shuttered rubber eyeguard protects the use from weapon recoil and prevents light from } \\
\text { escaping from the eyepiece. System magnification is } 3.7 \mathrm{x} \text { with a focus range from } 25 \mathrm{~m} \text { to infinity. Mechanical } \\
\text { dimensions are characterized by a length of } 24 \mathrm{~cm} \text {, a height of } 12 \mathrm{~cm} \text {, and a weight of approximately } 1.7 \mathrm{~kg} \text {. }\end{array}$ \\
\hline
\end{tabular}

\begin{tabular}{|l|}
\hline AN/PVS-10 Sniper Day/Night Weapon Sight \\
\hline $\begin{array}{l}\text { The AN/PVS-10 is an integrated day and night long range sight for the M24 sniper system. It offers flip-of-a- } \\
\text { lever } 24 \text { hour operation without the sacrifice of established shooting techniques of the M24 and M3A 10x sniper } \\
\text { scope. The AN/PVS-10 maintains the established } 2 \text { degree field of view, the same reticule pattern, the same } \\
\text { azimuth and elevation operation, the same rail mounting, and the same sniper cheek position on the weapons. } \\
\text { Upgraded features include variable tube gain and reticule illumination to maximize image contrast. These are } \\
\text { controlled by pushbuttons located on the top of the system accessible by either hand. A micro-switch prevents use } \\
\text { of the image tube when the system is in day mode. Standard systems are delivered with a special Generation III } \\
\text { manual gain tube, 8.5x objective lens and build in Weaver mount. This system is adaptable to the Remington } 700 \\
\text { series bolt action rifles and is available with optional 12x objective lens and afocal lens adapters. }\end{array}$ \\
\hline
\end{tabular}





\section{APPENDIX B}

\section{Currently Fielded Infrared Based Night Vision Devices}

\section{Short Range Thermal Sight}

Military applications of the uncooled sensor date back to the late 1970's. The Short Range Thermal Sight (SRTS) program, begun in 1985, developed a battery-operated weapon's sight for the U.S. Army, primarily to be used on the M-16 rifle. Systems continue in operation. The uncooled systems had demonstrated that they consistently outperform image intensification systems in battlefield situations like in Desert Storm.

\begin{tabular}{|c|c|c|}
\hline \multicolumn{3}{|l|}{ NIGHTSIGHT } \\
\hline \multicolumn{3}{|c|}{$\begin{array}{l}\text { The NIGHTSIGHT was developed from the Low Cost Uncooled Sensor Prototype (LOCUSP) program. The } \\
\text { NIGHTSIGHT works in full daylight or in the darkest of night, separating people and other objects from cluttered } \\
\text { background and foliage, greatly assisting continuous operations. It cuts through dust, smoke, haze, and other } \\
\text { battlefield obscurant. Because it emits no light or RF energy, its use cannot be detected. }\end{array}$} \\
\hline Weight: $\quad 3.1 \mathrm{lb}$ & Spectral Band: & $7.5-13.5 \Phi \mathrm{m}$ \\
\hline Size: $\quad 3.8^{\prime \prime} \mathrm{W}$ X $4.3 " \mathrm{H}$ X 12..9"L & Depth of Field: & $32 \mathrm{ft}$ to infinity \\
\hline Human Detection Distance: $2260 \mathrm{ft}$ & Field of View: & $15 \mathrm{E} \times 11.25 \mathrm{E}$ \\
\hline Start Up Time: $\quad<60$ seconds & Lens Focus: & Manual \\
\hline Operating Temperature: $-25 \mathrm{E}$ to $120 \mathrm{EF}$ & Image Polarity: & Black - hot or White - hot \\
\hline Power Requirements: 4 - 9 volt Batteries & Contrast Control: & Automatic \\
\hline Power Consumption: $\quad<6.0$ Watt & Brightness Control: & Manual \\
\hline Video Update Rate: $\quad 30 \mathrm{~Hz}$ & Video Output: & EIA RS-170/NTSC \\
\hline
\end{tabular}



ORNL/TM-2001/172

\title{
INTERNAL DISTRIBTUION
}

\author{
1. J. E. Hardy \\ 2. D. W. McDonald \\ 3. G. B. Singleton \\ 4-13. C. M. Smth \\ 14. J. D. White \\ 15.Central Research Library \\ 16. ORNL Laboratory Records-RC \\ 17. ORNL Laboratory Records-OSTI
}

\section{EXTERNAL DISTRIBUTION}

18-19. F. Downs, U.S. Navy Coastal Systems Station, 6703 W. Hwy 98, Code R41, Building 411, Special Operations, Panama City, Florida 32407-7001

20. J. Woods, U.S. Navy Coastal Systems Station, 6703 W. Hwy 98, Panama City, Florida 32407-7001.

21-22. R. Bordelon, 1636 Regulus Avenue, Virginia Beach, VA 23461.

23. B. Venanzi, 1636 Regulus Avenue, Virginia Beach, VA 23461. 